\title{
Mitochondrial Pathways, Permeability Transition Pore, and Redox Signaling in Cardioprotection: Therapeutic Implications
}

\author{
Claudia Penna, ${ }^{1,2}$ Maria-Giulia Perrelli, ${ }^{1,2}$ and Pasquale Pagliaro ${ }^{1,2}$
}

\begin{abstract}
Reperfusion therapy is the indispensable treatment of acute myocardial infarction (AMI) and must be applied as soon as possible to attenuate the ischemic insult. However, reperfusion is responsible for additional myocardial damage likely involving opening of the mitochondrial permeability transition pore (mPTP). A great part of reperfusion injury occurs during the first minute of reperfusion. The prolonged opening of mPTP is considered one of the endpoints of the cascade to myocardial damage, causing loss of cardiomyocyte function and viability. Opening of mPTP and the consequent oxidative stress due to reactive oxygen and nitrogen species (ROS/RNS) are considered among the major mechanisms of mitochondrial and myocardial dysfunction. Kinases and mitochondrial components constitute an intricate network of signaling molecules and mitochondrial proteins, which interact in response to stressors. Cardioprotective pathways are activated by stimuli such as preconditioning and postconditioning (PostC), obtained with brief intermittent ischemia or with pharmacological agents, which drastically reduce the lethal ischemia/reperfusion injury. The protective pathways converging on mitochondria may preserve their function. Protection involves kinases, adenosine triphosphate-dependent potassium channels, ROS signaling, and the mPTP modulation. Some clinical studies using ischemic PostC during angioplasty support its protective effects, and an interesting alternative is pharmacological PostC. In fact, the mPTP desensitizer, cyclosporine A, has been shown to induce appreciable protections in AMI patients. Several factors and comorbidities that might interfere with cardioprotective signaling are considered. Hence, treatments adapted to the characteristics of the patient (i.e., phenotype oriented) might be feasible in the future. Antioxid. Redox Signal. 00, 000-000.
\end{abstract}

I. Introduction

A. Acute myocardial infarction and reperfusion injury

B. Strategies to reduce injury

II. Mitochondrial Network

A. Important kinases of mitochondrial network involved in I/R injury and cardioprotection

1. GSK-3 $\beta$ in cell survival

2. PKC family in the I/R scenario and cardioprotection

3. The role of STAT-3 in cardioprotection by PreC and PostC

4. Pim-1 kinase in the I/R scenario and cardioprotection

5. Bcl-2 family: interaction with mitochondria

6. AMP-activated protein kinase, a metabolic regulator in health and disease

B. Important components of mitochondria in the network involved in $\mathrm{I} / \mathrm{R}$ and cardioprotection

1. Mitochondrial permeability transition pore

2. Putative mitochondrial ATP-sensitive potassium channels

3. Mitochondrial Cx43

4. Mitochondrial uncoupling proteins

C. ROS/RNS: from mitochondria to activation of kinases of the network

Reviewing Editors: Enrique Cadenas, Amadou Camara, Anonymous, Nikolaos Frangogiannis, David Gutterman, Kristina Leuner, Simona Rapposelli, and Jun Ren

\footnotetext{
${ }^{1}$ Department of Clinical and Biological Sciences, University of Turin, Orbassano, Italy.
}

${ }^{2}$ National Institute of Cardiovascular Research (INRC), Bologna, Italy. 
III. Role of Mitochondria in Acute I/R Injury

A. mPTP opening in acute $\mathrm{I} / \mathrm{R}$

1. Consequences of prolonged mPTP opening

B. Prevention of prolonged mPTP opening

C. Transient opening of $\mathrm{mPTP}$ can be protective

D. Chronic ischemia and mitochondria

IV. Cardioprotective Strategies Targeting Mitochondria in Acute I/R Injury

A. PreC and PostC

B. Redox signaling and acidosis in early reperfusion

1. Detrimental effects of excessive ROS

2. Beneficial effects of redox signaling and acidosis

V. Timing and Targets of ROS Signaling in Cardioprotection

A. ROS/RNS signaling may be modulated by antioxidants

B. Role of SNO in regulating mitochondrial function in I/R and cardioprotection

VI. Preservation of Functional and Morphological Integrity of Mitochondria by PostC

VII. Summary of PreC and PostC Pathways United at Reperfusion

VIII. The Second Window of Protection

IX. Autophagy and Mitophagy

X. Comorbidities, I/R, and Cardioprotection

A. Mitochondria and MS

1. Cardioprotection in MS

B. Cardioprotection in Aging

C. Cardioprotection in hypertension and hypertrophy

XI. Transition to the Clinical Setting

A. The possible reasons of variable outcomes with ischemic PostC

B. Pharmacological PostC

C. Features of a successful cardioprotective approach in early reperfusion

XII. Executive Summary and Conclusions

\section{Introduction}

$\mathbf{T}$ HIS REVIEW DESCRIBES the role of mitochondria in the cardioprotection by preconditioning (PreC) and postconditioning (PostC): two cardioprotective maneuvers that target mitochondria. Before providing details on the conditionings, we briefly introduce the ischemia/reperfusion (I/R) injury, the cardioprotective strategies, and their molecular pathways. Moreover, we describe in the details some factors involved in the cross-talk between the cytosol and mitochondria (mitochondrial network) in different scenarios, including I/R and cardioprotection. Besides the classic players (protein kinase $\mathrm{C}[\mathrm{PKC}]$, glycogen synthase kinase-3 $\beta$ [GSK$3 \beta]$, mitochondrial ATP-sensible $\mathrm{K}^{+}\left[\mathrm{mK}_{\mathrm{ATP}}\right]$ channels, etc. $)$, we also describe the role of relatively new cardioprotective mitochondrial factors such as signal transducer and activator of transcription-3 (STAT-3) and Pim-1. Among important elements of the cross-talk between elements of the mitochondrial network, we consider reactive oxygen and nitrogen species (ROS/RNS), which are mainly produced by mitochondria. Then, we describe how the considered elements come into play together with other factors in the I/R scenario, in the regulation of mitochondrial function, and in the cardioprotective pathways that converge on mitochondria. The main protective pathways considered are the so-called reperfusion injury salvage kinases (RISKs) and survivor activating factor enhancement (SAFE). Since mitochondria are the main source of ROS in the cardiomyocytes, emphasis is given to ROS signaling in cardioprotection. We also briefly consider the role of mitochondria in autophagy (a tightly regulated cellular housekeeping process that may confer increased re- sistance to I/R injury) and in the so-called second window of protection (SWOP) induced by PreC. Before taking into account the clinical translation of conditioning strategies, we discuss the role of aging and some comorbidities, which modify the mitochondrial function and the cardioprotective outcome. In considering transition to clinic, possible pitfalls of ischemic PostC and possible aspects that should be considered for a successful cardioprotective approach in early reperfusion with pharmacological PostC are discussed.

\section{A. Acute myocardial infarction and reperfusion injury}

Acute myocardial infarction (AMI) continues to be a major cause of morbidity and mortality, and infarct size is the major determinant of patients' prognosis. Millions of people worldwide each year die from AMI. With the in-hospital mortality rate of 5\%-6\% among ST segment elevation myocardial infarction (STEMI) patients and about $4 \%$ among non-ST segment elevation myocardial infarction (NSTEMI) patients, AMI remains the first cause of death in Western countries and is the leading cause of chronic heart failure and cardiovascular diseases (302). All this happens despite the incredible improvements in the care of patients with AMI in recent years. In fact, prompt myocardial ischemic reperfusion is a very good strategy to reduce the infarct size and reduce all manifestations of postischemic injury resulting in improved outcomes. The culprit coronary artery can be opened by percutaneous coronary intervention (PCI) or fibrinolytic agents, with or without stenting. Although early reperfusion is the only way to save an ischemic organ, reversible and irreversible organ damage occurs during the early moments of reperfusion (that is reperfusion injury). 
Myocardial ischemia is characterized by severe hypoxia, acidosis, energy depletion, and ion homeostasis alterations leading to cardiac dysfunction and, ultimately, to cell death. Mitochondria are abundant in cardiomyocytes, and they use oxygen as the principal substrate, so that it is not surprising that they play a role of protagonist in the I/R scenario during hypoxia/ischemia/reoxygenation. Mitochondria also play a pivotal role in ion homeostasis. In fact, the key events that occur in cardiomyocytes during I/R are imbalanced and altered exchange of ions. These are precipitated by the metabolic and chemical changes of ischemia, which then triggers mitochondrial dysfunction during reperfusion. Therefore, mitochondria play a critical role in the regulation of cardiac function in both health and disease, and are also increasingly recognized as end effectors for various cardioprotective signaling pathways. Therefore, understanding the role of mitochondria in this scenario is a requisite to find an appropriate therapeutic approach.

Myocardial injury that follows ischemia and reperfusion is, of course, related to the duration of ischemia. Successful reperfusion has been shown to dramatically reduce the infarct size; the sooner it is performed, the greater the amount of saved myocardium. However, it has also been seen that a large component of the damage takes place during the first minutes of reperfusion when an amplification of ischemic injury or additional damage occurs $(12,115,116,256,396)$. Intracellular $\mathrm{Ca}^{2+}$ overload, inadequate resynthesis of adenosine triphosphate (ATP), oxidative stress by ROS, and loss of membrane phospholipids have been suggested as contributing to reperfusion injury $(21,285,383)$. Therefore, both ischemia and reperfusion contribute to organ damage. Reperfusion injury includes arrhythmias, transient mechanical dysfunction of the heart or myocardial stunning, microvascular damage, and no reflow, as well as inflammatory responses. In reperfusion, cell death can occur by apoptosis, necrosis, and autophagy [the reader is redirected to extensive review on this topic (e.g., 115, 260, 269, 270, 290, 347, and 369)]. However, in contrast to necrosis and apoptosis, which inevitably lead to cell death, autophagy is not simply a destructive phenomenon, but under certain conditions, autophagy can be considered a protective mechanism against I/ R injury (290, 347). The vulnerability to $I / R$ injury is likely to be heavily influenced by the autophagic control of protein and organelle quality, including mitochondria. For these reasons, autophagy is briefly discussed in a dedicated section of this review (see section IX).

A relevant role in exacerbating myocardial injury by favoring mitochondrial permeability transition pore (mPTP) opening is played by ROS, which are generated at various sites in the cell and within mitochondria. The control of $\mathrm{MPTP}$ opening and ROS generation appears to modulate the I/R injury. To control mitochondrial function, a plethora of reactions occurs outside and within the mitochondria themselves. In Figure 1 are reported some of the elements in play, which will be responsible for cell death via mPTP opening: Figure 1A displays the balance of factors controlling $\mathrm{mPTP}$ opening in ischemia; Figure 1B illustrates the conditions favoring the $\mathrm{mPTP}$ opening and the main consequences upon reperfusion.

\section{B. Strategies to reduce injury}

Since recent data indicate that different forms of cell death are probably correlated $(115,116)$, the best strategy for developing cardioprotective agents is not to define the mode of cell death and its proportion occurring during I/R, but to identify mediators active in all forms of cell death. In this context, mitochondria have emerged as the relevant targets. Interestingly, the change in the mitochondrial membrane permeability occurring in early reperfusion appears to be among the most important regulators of all forms of cell death.

The timing of the reperfusion injury occurrence explains the efficacy of the PostC interventions in limiting the infarct size and in ameliorating the mechanical recovery of the heart $(174,329)$. The involved factors and the consequences of PostC are discussed in the following sections. Timing of cardioprotective interventions is of paramount importance for limiting $\mathrm{mPTP}$ opening. In fact, both ischemic PreC and ischemic
FIG. 1. Schematic representation of factors controlling mitochondrial permeability transition pore (mPTP) open probability, during ischemia (A) and during reperfusion (B). Ischemia-induced intracellular acidosis may shift the equilibrium toward the closed state. Upon reperfusion, the recovery of $\mathrm{pH}$ toward neutral values facilitates the concomitant elevation of matrix $\left[\mathrm{Ca}^{2+}\right]$ and reactive oxygen and nitrogen species (ROS/ RNS) formation in promoting $\mathrm{mPTP}$ opening. For other acronyms, see the list of $\mathrm{Ab}$ breviations Used.
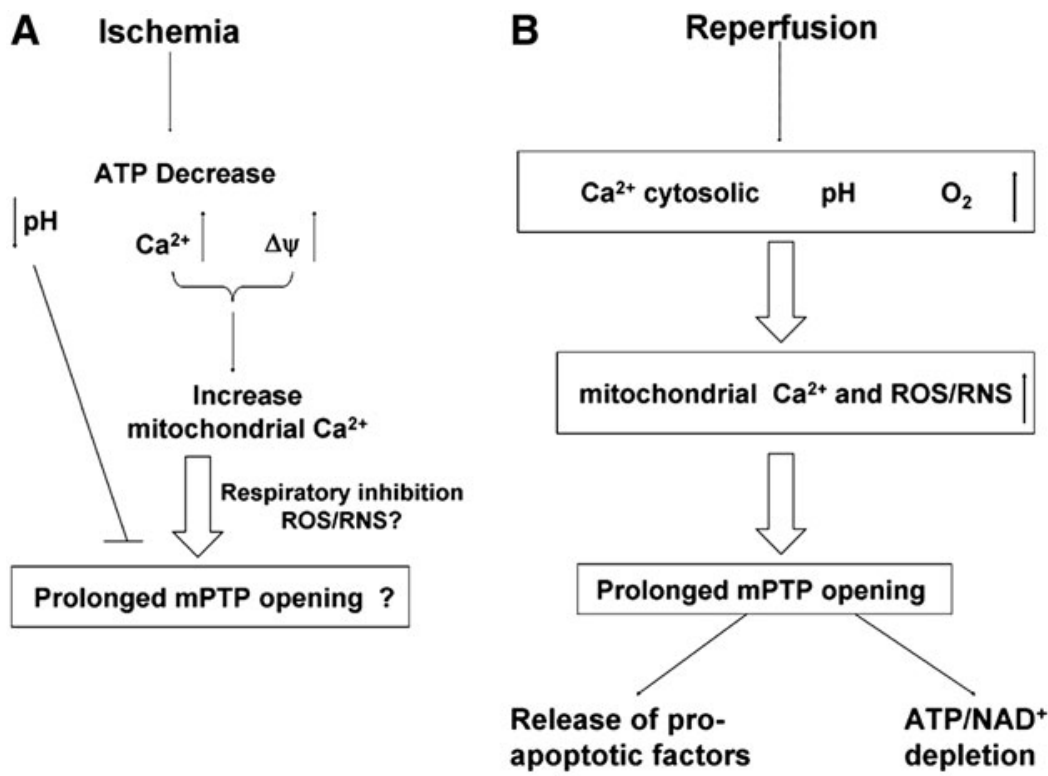
PostC have been demonstrated to significantly attenuate I/ $R$ injury. Although PreC can be obtained with one or more brief coronary occlusions (a few min each) before the infarcting ischemia, PostC may be performed with one or more brief occlusions (a few seconds each), starting immediately after the end of the ischemia in animals and humans (e.g., 61, 133-136, 174, 260, 329, and 333). Moreover, PreC initiates an immediate protective response (early PreC) and 12-24h later, a more modest protection against the infarct size (SWOP), which will be considered briefly in the section VIII (Fig. 2).

The necessity of the early intervention for PostC to be protective has emphasized the importance of reperfusion in inducing I/R injury. Since the PostC maneuvers are not protective if they are carried out after the first minutes of reperfusion $(260,392)$, this period seems to be confirmed as the interval when most damage takes place. The main advantage of PostC with regard to PreC consists in the more frequent possibility of clinical application. In fact, due to the unpredictability of an ischemic event, PostC can be utilized with reperfusion procedures, which may be under the control of physicians $(212,333)$.

Cardioprotection may be due to passive mechanisms, which are responsible for a reduction in the reperfused heart of (i) endothelial cell activation and dysfunction; (ii) neutrophil activation and accumulation; (iii) tissue ROS generation;

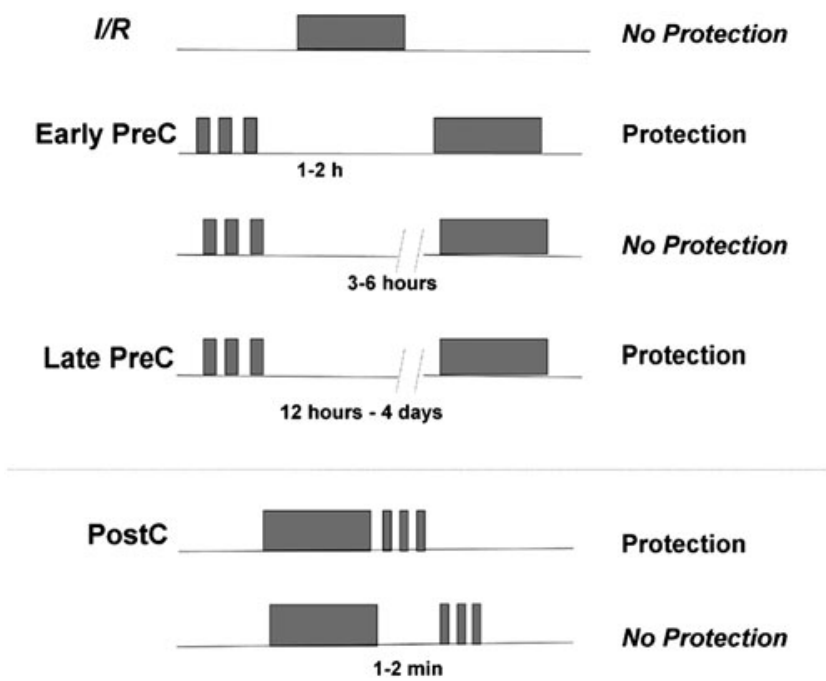

FIG. 2. Schematic diagrams illustrating the protocols of preconditioning (PreC) and postconditioning (PostC). PreC is triggered by short periods of ischemia (a few minutes) applied before the onset of a sustained period of ischemia. PreC consists of two chronologically and pathophysiologically distinct phases: an early phase (Early PreC), which develops very quickly, within a few minutes from the exposure to the stimulus, but lasts only $1-2 \mathrm{~h}$ [this is the phenomenon originally described by Murry et al. (239)], and a late phase (Late PreC), that is the so-called second window of protection (SWOP), which develops more slowly (requiring $6-12 \mathrm{~h}$ ), but lasts much longer (3-4 days) $(35,37,342,348)$. PostC is triggered by short periods of ischemia (a few seconds) applied immediately after the end of the sustained ischemia [this is the phenomenon originally described by Zhao et al. (393)]. PostC maneuvers are not protective if they are carried out after the first minutes of reperfusion (260, 392). For other acronyms, see the list of Abbreviations Used. and (iv) microvascular injury and tissue edema $(269,368,369)$. However, cardioprotection by PreC and PostC triggers also active mechanisms, which consist of the activation of intracellular signaling pathways that lead to limitation of cell death $(268,269,369,393)$. These signaling pathways initiate before sustained ischemia for ischemic PreC, but importantly, they initiate also at the very start of reperfusion for both PreC and PostC. In fact, both ischemic PreC and PostC reduce myocardial damages due to ischemia and reperfusion.

While in ischemic PreC, the brief cycles of protective I/R are employed before the sustained infarcting ischemia; in ischemic PostC, they are employed at the onset of reperfusion after sustained ischemia; however, both ischemic PreC and PostC are protective phenomena both targeting reperfusion (Fig. 3); as such, they share certain signaling elements in experimental analyses (137, 144, 145, 269, 382). At reperfusion, there are three populations of cells: (i) those that were killed by sustained ischemia, (ii) those that had sublethal injury and will survive, and (iii) those that are alive, but will die mainly from mPTP opening. Both PreC and PostC target this last group of cells. In fact, it is now thought that the actual PreC protection occurs in the reperfusion, rather than the ischemic phase, and repopulation of membrane receptors and activation of multiple kinases are critical events $(61,64)$. In PreC, we recognize a trigger phase in which adenosine, bradykinin, opioids, and possibly, other surface receptors couple through multiple pathways to activate $\mathrm{mK}_{\mathrm{ATP}}$ and PKC. In the ischemic phase, PKC may act as a memory, and in reperfusion phase, signal transduction pathways act to prevent mPTP opening (Fig. 3A). Also, PostC protects because it maintains acidosis during early reoxygenation (167), which inhibits mPTP formation and allows ROS signaling (Fig. 3B) that gives the heart enough time to activate signaling pathways, and thus to precondition itself against reperfusion injury.

The signaling elements recruited by these cardioprotective phenomena, especially those of the PreC trigger phase, have been extensively studied. It has become clear that different pathways finally target the mitochondria, and that preservation of mitochondrial structure and function is central for cardioprotection [for reviews see $(27,81,260)]$. In other words, the mitochondrial structure and function are preserved by the cardioprotective maneuvers, because they are able to affect the cross-talk between cytosolic and mitochondrial elements. Besides the survival kinases of the RISK (Akt/ERK1/ $2 / \mathrm{GSK}-3 \beta$ ) and protein kinase G (PKG)/PKC pathways, also SAFE (TNF- $\alpha /$ JAK/STAT-3) pathways, as well as the AMP-activated protein kinase (AMPK), Pim-1, and B-cell lymphoma-2 (Bcl-2) family may contribute to preserve the mitochondrial function and to avoid cell death.

Actually, the majority of the mitochondrial proteins is synthesized in the cytosol and has to be imported $(52,100)$. This import is influenced by protective maneuvers. Also, mitochondrial factors/products (e.g., ATP, cytochrome c [Cyt c], and $\mathrm{ROS}$, mainly hydrogen peroxide $\left[\mathrm{H}_{2} \mathrm{O}_{2}\right]$ ) may be released in to the cytosol, affecting cell life and death. Both the import toward and the release from mitochondria are influenced by stressors like I/R and by cardioprotective maneuvers like PreC and PostC (e.g., 27, 137, and 139). Several of the aforementioned signaling and mitochondrial components take part to this cross-talk between the cytosol and mitochondria, constituting a mitochondrial network. Here, we will 
FIG. 3. Current theory for PreC (A) and for PostC (B). In the PreC, we recognize a Trigger phase: adenosine (Ade), bradykinin (BK), and other surface receptors couple through multiple pathways to activate PKC and possibly other kinases; ischemic phase (sustained ischemia): kinases act as a memory; reperfusion phase (early reperfusion): signal transduction pathways act to prevent $\mathrm{MPTP}$ opening. In the PostC, intermittent PostC ischemias do not allow a rapid recovery of $\mathrm{pH}$ in early reperfusion, thus keeping mPTP closed and allowing the heart to precondition itself. It has been suggested that low $\mathrm{pH}$ in early reperfusion plays a role also in PreC (139). For other acronyms, see the list of $\mathrm{Ab}$ breviations Used.

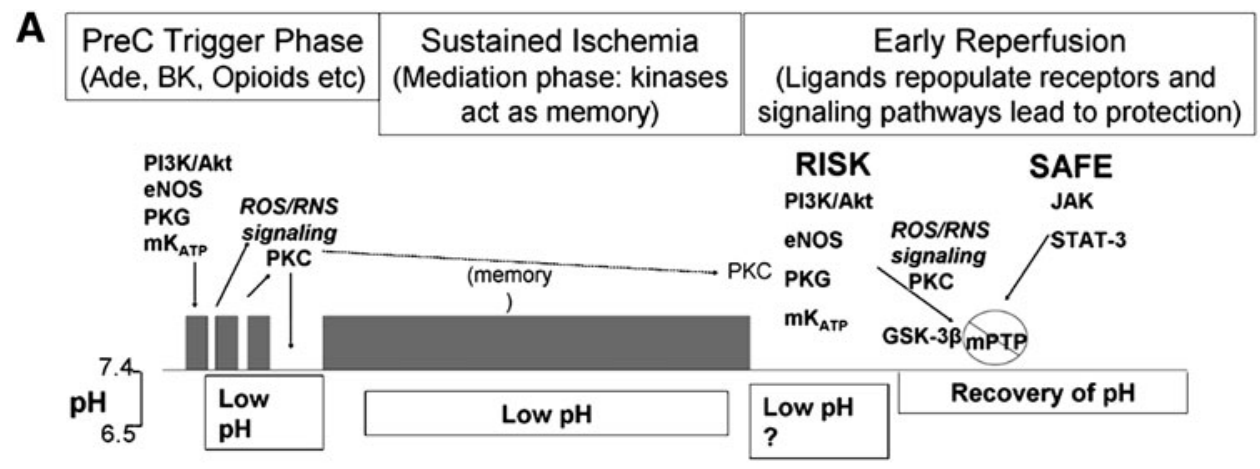

B

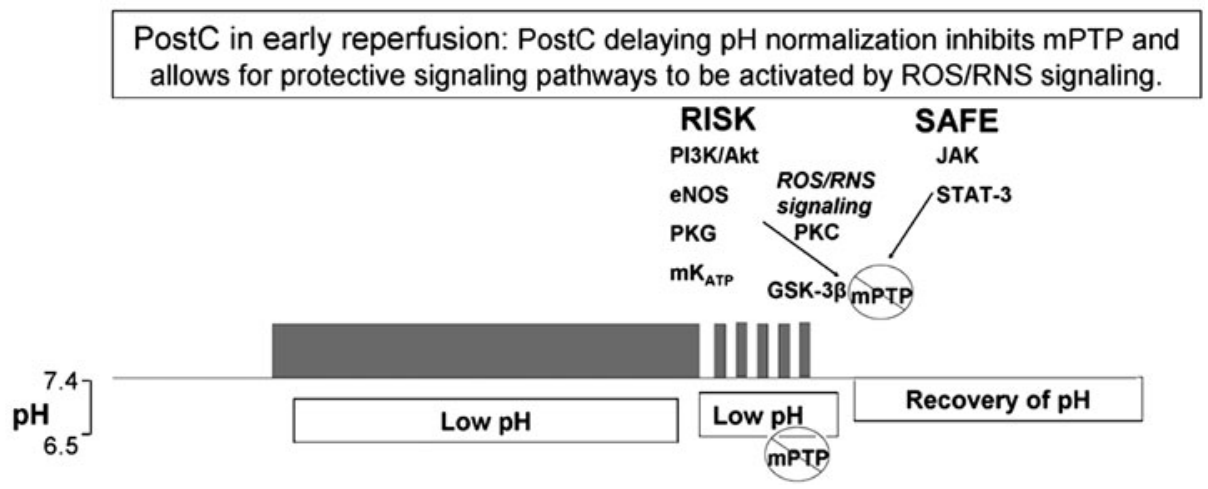

consider some of these elements, which are modified by I/R and cardioprotective maneuvers.

\section{Mitochondrial Network}

Due to space constraint, here, we consider only the most frequently studied elements of the complex network of signaling molecules between the cytosol and mitochondria (Fig. 4).

\section{A. Important kinases of the mitochondrial network involved in $I / R$ injury and cardioprotection}

The heart should constantly adjust energy production to energy supply and utilization, and is a high-energy consumer. For this reason, the heart greatly depends on oxidative metabolism for adequate energy production and on efficient energy-transfer systems. Therefore, the function of mitochondria is finely regulated by intrinsic (e.g., mPTPs and $\mathrm{mK}_{\mathrm{ATP}}$ channels) and extrinsic factors (e.g., GSK-3 $\beta$ and PKC). Before taking into account the role of mitochondria in the scenario of $I / R$, it is useful to consider how some of these factors are involved in the regulation of mitochondrial functions in physiological conditions and during stress.

1. GSK-3 $\beta$ in cell survival. GSK-3 $\beta$ is important for glycogen metabolism, but it is also involved in gene expression, and cell survival. For instance, GSK- $3 \beta$ phosphorylates pyruvate dehydrogenase (PDH), creating an energy deficit in the cell (158), and it also phosphorylates the docking site of hexokinases (HKs) to voltage-dependent anion channel (VDAC), facilitating the induction of outer mitochondrial membrane (OMM) permeabilization and subsequent cell death (50). The GSK-3 $\beta$ level may change in models of hypertrophy (279), and its activity is mainly regulated via phosphorylation: the phosphorylation of GSK-3 $\beta$ at serine 9 renders the protein inactive.

Pharmacological inhibition of GSK-3 $\beta$ (i.e., GSK-3 $\beta$ phosphorylation) either before ischemia or at reperfusion may reduce I/R injury. In fact, it seems that both ischemic PreC and PostC are associated with enhanced GSK-3 $\beta$ phosphorylation/inhibition in rodent hearts $(117,236,274,356)$.

In the context of cardioprotection, it has been proposed that activation of both the phosphatidylinositol 3-kinase (PI3K)/ protein kinase $\mathrm{B}$ (PKB or Akt) and the extracellular signalregulated kinase (ERK) $1 / 2$ projects onto downstream kinases, including GSK-3 $\beta$, and ultimately to the OMM to affect cellular survival $(133,134,137)$. In fact, pharmacological inhibition of GSK-3 $\beta$ phosphorylation clearly abolishes cardioprotection $(185,186)$. However, transgenic approaches have revealed ambiguous results not only for GSK-3 $\beta$ but also for GSK- $3 \alpha$; in fact,

(i) in transgenic mice in which serine 9 is replaced by alanine to render GSK-3 $\beta$ insensitive to phosphorylation, infarct size reduction by ischemic PostC is lost (117);

(ii) in mice with a targeted knocking of noninhibitable GSK- $3 \alpha$ and GSK-3 $\beta$, infarct size reduction by ischemic PreC or PostC is preserved (248);

(iii) in knockout $(\mathrm{KO})$ mice for GSK-3 $\alpha$, apoptotic cells in the border zone of the infarction were increased, suggesting that the $\alpha$-isoform acts to limit ischemic injury (210);

(iv) using RNA interference in neonatal rat cardiomyocytes, it was concluded that the knockdown of GSK$3 \beta$ was protective against ischemic injury, but that the knockdown of GSK- $3 \alpha$ was not (185).

These apparently contradictory findings may be related to different genetic strains and models. However, also PostC in 


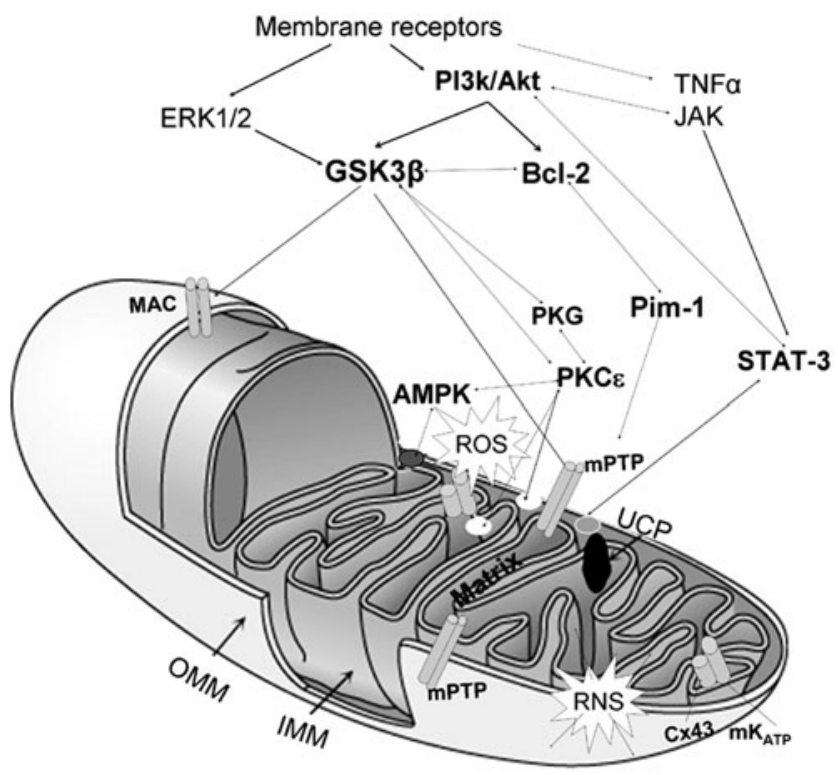

FIG. 4. Elements of the mitochondrial network. Among cytosolic and mitochondrial elements, exists a very complex network of interactions. The interactions of this network are better disentangled in the other figures. The double arrows do not indicate direct interaction among elements, but indicate that there is a cross-talk among the various elements. It is likely that Akt can modulate almost all the downstream elements. It is also likely that some of the elements and kinases migrate from outside to inside the mitochondria. As discussed in the text, most of the information relative to interplay of this network derives from our laboratory and the laboratories of J. Vinten-Johansen, D. Hausenloy \& D. Yellon, G. Heusch \& R. Schulz, D. Garcia-Dorado, M. Choen \& J.M. Downey, and E. Murphy \& C. Steenbergen, and from F. Di Lisa and A. Halestrap's laboratories. In particular, the interconnections between elements, as for example, PKC and PKG role, are matter of debate among some of these laboratories. We will focus on the more common interrelations of these elements in ischemia/reperfusion and cardioprotection scenarios. ROS and RNS are second messengers of life or death that may be produced by mitochondria (see text for further explanation). Of course, each of the considered elements has multiple targets that are not considered in this figure and in the text. For other acronyms, see the list of Abbreviations Used.

pigs did not increase postischemic GSK-3 $\beta$ phosphorylation over that induced by reperfusion (328). Overall, these studies support a highly variable role of both GSK-3 $\alpha$ and GSK-3 $\beta$ for cardioprotection.

Further studies are necessary to understand the conditions affecting its protective role. Nevertheless, when protective, the effects of GSK-3 $\beta$ involve mitochondrial proteins and structures, for example, inhibition of mPTP opening and control of mitochondrial adenine nucleotide transporter (ANT) in the inner mitochondrial membrane (IMM) through the OMM $(117,186,246,247)$. Since GSK-3 $\beta$ is mainly localized in the cytosol, but targets mitochondrial proteins, attention has been directed to the presence and action of GSK-3 $\beta$ within mitochondria. Increases in the content of total and phosphorylated GSK- $3 \beta$ in mitochondria of isolated rat hearts subjected to I/R and an enhancement of protein-protein in- teraction between GSK-3 $\beta$ and VDAC or ANT have been observed (239). At present, it is not at all clear how proteins residing in the IMM (e.g., ANT and PDH), or even in the matrix as in the case of cyclophilin D (Cyp-D) in cancer cells (299), can be targeted and modulated by this cytosolic protein kinase. Nevertheless, phosphorylated GSK- $3 \beta$ inhibits mPTP opening likely by multiple mechanisms, including preservation of HK in the mPTP complex and prevention of interaction of Cyp-D with ANT $(50,137)$.

In mitochondria, isolated from rat cardiomyocytes, the phospho-GSK-3 $\beta$-to-total GSK-3 $\beta$ ratio correlates with the $\mathrm{Ca}^{2+}$ concentration needed to induce mPTP opening (231). It has been, thus, suggested that the level of mitochondrial GSK$3 \beta$ phosphorylation at reperfusion is a determinant of the threshold for mPTP opening (231). Accordingly, cardioprotection by ischemic PostC was associated with enhanced mitochondrial contents of total and phosphorylated GSK-3 $\beta$ and with an increased phospho-/total GSK-3 $\beta$ ratio in ex vivo rat hearts (274). However, translocation/phosphorylation of GSK-3 $\beta$ to/in mitochondria was not affected by ischemic PreC in isolated rat hearts (59).

In summary, it seems that in ischemic PreC and PostC, the obligatory role of GSK-3 $\beta$ for cardioprotection is still controversial and possibly species specific $(117,153,185,248,328$, $371,382)$. Nevertheless, further studies are needed to specifically characterize the role of mitochondrial GSK-3 $\beta$ in cardioprotection and to distinguish between the effects of cytosolic and mitochondrial GSK-3 $\beta$.

2. PKC family in the $\mathrm{I} / \mathrm{R}$ scenario and cardioprotection. The serine/threonine PKC family was first identified as intracellular receptors for the tumor-promoting agents phorbol esters. There are multiple isoforms of PKC that function in a wide variety of biological systems. The conventional PKC isoforms (PKC- $\alpha, \beta 1, \beta 2$, and $\gamma$ ) are activated by phosphatidylserine (PS), calcium, and diacylglycerol (DAG), or phorbol esters such as phorbol 12-myristate 13-acetate (PMA), whereas novel PKCs (PKC- $\delta, \varepsilon, \theta$, and $\eta$ ) are activated by PS, DAG, or PMA, but not by calcium. The atypical PKCs (PKC- $\zeta$ and $\imath / \lambda$ ) are not activated by calcium, DAG, or PMA; PKC $\lambda$ is for mouse; the human homolog is termed $\mathrm{PKC}_{l}$.

Cardioprotective mechanisms involve the activation of several membrane receptors of agonists such as adenosine, bradykinin, and opioids. All these agonists eventually target PKC: adenosine through the activation of phospholipase C, and the other agonists through a more complex pathway that includes serial activation of PI3K/Akt, nitric oxide synthase (NOS), guanylyl cyclase, PKG, and opening of $\mathrm{mK}_{\mathrm{ATP}}$ channels, and finally production and release of ROS, which target PKC within and outside mitochondria $(66,67,113,296)$. Mitochondrial ROS are represented by superoxide anion $\left(\mathrm{O}_{2}{ }^{-\bullet}\right)$ from which can derive its products $\mathrm{H}_{2} \mathrm{O}_{2}$ and hydroxyl radical $\left(\mathrm{OH}^{\bullet}\right)$. Actually, $\mathrm{H}_{2} \mathrm{O}_{2}$ might be the only oxidant released from mitochondria to the cytosol in physiological conditions; the negatively charged $\mathrm{O}_{2}{ }^{-} \bullet$ does not permeate the lipid bilayer of biological membranes. However, it is able to pass through the pore of anion channels (Fig. 5), and in some cases, it may play a role as such or after a reaction with nitric oxide $\left(\mathrm{NO}^{\bullet}\right)$ to form peroxynitrite $\left(\mathrm{ONOO}^{-}\right)(8,9,79,81,205,399)$.

The PKC isoforms mainly studied in $\mathrm{I} / \mathrm{R}$ and cardioprotection are $\mathrm{PKC} \alpha, \mathrm{PKC} \delta$, and $\mathrm{PKC} \varepsilon$. After I/R, PKC $\delta$ enhances and $\mathrm{PKC} \varepsilon$ reduces irreversible myocardial injury in rodents. 


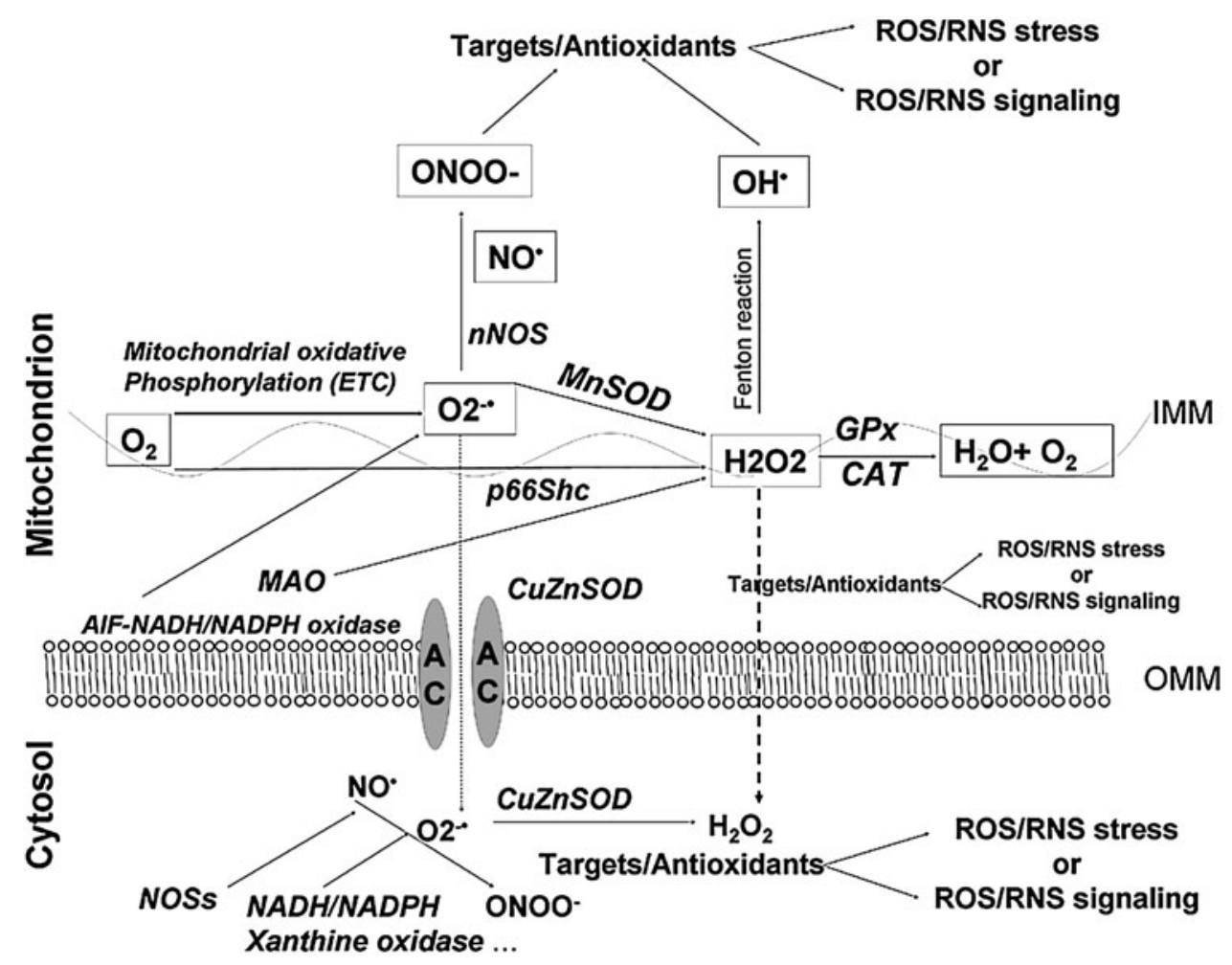

FIG. 5. ROS/RNS are products of multiple enzymes and reactions within and outside the mitochondria. The immediate product of multiple enzymes, including the enzymes of mitochondrial oxidative phosphorylation, may be the superoxide anion $\left(\mathrm{O}_{2}{ }^{-\bullet}\right)$, mainly generated at complexes I and III of the electron transport chain (ETC); however, due to spontaneous and enzymatic formation by p66Shc and dismutation by SOD, hydrogen peroxide $\left(\mathrm{H}_{2} \mathrm{O}_{2}\right)$ is also rapidly generated. While $\mathrm{H}_{2} \mathrm{O}_{2}$ easily cross the biological membranes, the negatively charged $\mathrm{O}_{2}{ }^{-\bullet}$ does not permeate the lipid bilayer of membranes. However, $\mathrm{O}_{2}{ }^{-\bullet}$ is able to pass through the pore of anion channels (ACs), such as voltage-dependent mitochondrial $\mathrm{AC}^{-\mathrm{H}_{2} \mathrm{O}_{2}}$ can be transformed to the more dangerous $\mathrm{OH}^{\bullet}$. Superoxide anion can react with $\mathrm{NO}^{-}$to generate $\mathrm{ONOO}^{-}$and other $\mathrm{RNS}^{2}$ Either redox stress or redox signaling can occur on both sides of membrane. However, it must be borne in mind that the switch from redox signaling to redox stress (within and outside mitochondria) does not depend only by the type of ROS/RNS but also on the amount of ROS/RNS and the vicinity of targets and antioxidants. Several isoforms of SOD degrade $\mathrm{O}_{2}^{-}{ }^{-}$to $\mathrm{H}_{2} \mathrm{O}_{2}$, including manganese (MnSOD) in the matrix and copper/zinc SOD (CuZnSOD) in the intermembrane space and cytosol. In the matrix, $\mathrm{H}_{2} \mathrm{O}_{2}$ is further detoxified to water primarily by glutathione peroxidase (GPX) and by catalase (CAT). The thickness of dotted lines represents the ability to diffuse through the membrane. For other acronyms, see the list of Abbreviations Used.

$\mathrm{PKC} \alpha$ is the isoform involved in $\mathrm{I} / \mathrm{R}$ injury and protection from it in pigs (328).

Activation of PKC $\delta$ induces cell death (apoptosis and oncosis) through the regulation of mitochondrial function. It has been suggested that increased levels and catalytic activity of PKC $\delta$ in the mitochondrial fraction are associated with hyperphosphorylation of PDH (58). This and other mechanisms may be involved in PKC $\delta$-induced cell death, whereas its degradation has been involved in protective mechanisms $(55,57)$.

Activation of $\mathrm{PKC}_{\varepsilon}$ protects mitochondrial function and diminishes cell death. Upon a PreC stimulus, PKC $\varepsilon$ translocates from the cytosol to the particulate fraction, but also to the mitochondria (251). Also, the cardioprotection by ischemic PostC was lost in isolated rat hearts treated with aspecific and specific PKC $\varepsilon$ inhibitors $(276,388)$.

Importantly, as said, PKC $\varepsilon$ may be activated by ROS signaling $(260,288,341)$. Within mitochondria, PKC $\varepsilon$ is located in the intermembrane space, and it is associated with the IMM, but the presence of a second PKC $\varepsilon$ pool within the matrix has been suggested (66). PKC $\varepsilon$ is imported into mitochondria by heat shock protein 90 (Hsp90) and outer membrane translo- case 20 (Tom20), playing a role in cardioprotection from I/R injury (42). Within mitochondria, PKC $\varepsilon$ has several targets, including $\mathrm{mK}_{\mathrm{ATP}}$ channels and aldehyde dehydrogenase (ALDH). The latter contributes to cardioprotection by inhibition of toxic aldehyde formation (56). It has been reported that PKC $\varepsilon$ phosphorylates VDAC when HKs (I and II) are overexpressed. In fact, $\mathrm{HKs}$ play a pivotal role in promoting cell survival by binding specifically to the VDAC, whereas HK removal triggers cell death (50). PKC $\varepsilon$ also targets mitogen-activated protein kinases (MAPKs) and ERK, forming a module that induces the phosphorylation and inactivation of the pro-apoptotic protein Bad (9).

In summary, PKCs are important for the I/R and for the cardioprotection by ischemic PreC and PostC. In particular, mitochondrial $\mathrm{PKC} \varepsilon$ contributes to cardioprotection by regulating the activity of several mitochondrial proteins (e.g., VDAC, connexin-43 [Cx43], $\mathrm{mK}_{\mathrm{ATP}}$ channels, and ALDH). On the contrary, $\mathrm{PKC} \delta$ may play a pivotal role in I/R injury.

3. The role of STAT-3 in cardioprotection by PreC and PostC. The STAT family consists of seven identified 
members (STAT-1, STAT-2, STAT-3, STAT-4, STAT-5A, STAT-5B, and STAT-6), all expressed in the heart. STATs are tyrosine phosphorylated by activated Janus kinases (JAKs), which allow them to form homodimers or heterodimers that translocate to the nucleus, resulting in gene transcription. In fact, the activation of the JAK/STAT pathway plays a pivotal role in the expression of stress-responsive genes. While the activation of STAT-1 is associated with apoptosis, the activation of STAT-3 affords cardioprotection [for reviews see (28, $107,156,258)]$. The localization of STAT proteins in the heart is not restricted to the cytosol and the nucleus; recently, they have also been identified in cardiomyocyte mitochondria (29, 373). The majority of studies investigating the role of STAT proteins in cardiac function have focused on STAT-3, which plays a role in apoptosis, heart failure, hypertrophy, postpartum cardiomyopathy, and I/R injury $(28,34,155,352)$. Recently, a role for STAT- 5 activation in cardioprotection has been evidenced in humans (148).

The cardioprotective role of STAT-3, which activates expression of antiapoptotic, antioxidative genes, has been previously reviewed (e.g., 28, 34, and 137). In the present section of the review, only STAT-3 targeting mitochondria will be considered. Effects of STAT-3 on the nucleus in relation to cardioprotection will be considered in the section VIII, dedicated to SWOP.

Besides RISK (Akt/ERK/GSK-3 $\beta$ ) and PKG/PKC pathways in both PreC and PostC, another cardioprotective pathway involves tumor necrosis factor (TNF)- $\alpha$, sphingosine, and the JAK/STAT-3 system, which Lecour has called the SAFE pathway (212). SAFE may interact with the other protective pathways, and a cross-talk between PI3K/Akt and STAT-3 has been proposed (137). Therefore, STAT-3 acts as both signaling molecules and transcriptional regulators, transducing stress signals from the plasma membrane to both the nucleus and to the mitochondria, predominantly in the mitochondrial matrix (29). The important role of STAT-3 in cardioprotection may be in part mediated by its effects on mitochondrial respiration and MPTP opening. In fact, inhibition of mitochondrial STAT-3 reduces ADP-stimulated respiration of myocardial mitochondria and calcium-induced mPTP opening (29). Effects of STAT-3 on mitochondrial respiration have been extensively reviewed in a recent article of Szczepanek et al. (344). It seems that STAT-3 is necessary for the optimal activity of complexes I and II of the electron transport chain (ETC). STAT-3-deficient embryos die because of defects of the visceral endoderm. To overcome the problem of embryonic lethality, cardiomyocyte-specific STAT-3-KO mice were generated (181). STAT-3 deletion in cardiomyocytes attenuates integrated respiration due to a decrease in the enzymatic activities of complexes I and II (373). The overexpression of mitochondrial-targeted STAT-3 in mice also results in a partial inhibition of electron transport at complexes I and II that does not affect baseline mitochondrial membrane potential nor enhance the production of ROS. It seems, however, that this inhibition may attenuate the production of ROS from complex I during I/R. In fact, the targeting of transcriptionally inactive STAT-3 to mitochondria may attenuate injury to mitochondria during cell stress, resulting in decreased production of ROS and less release of $\mathrm{Cyt}$ c from mitochondria (343). Since cyclosporine A (CsA) reduces the infarct size to a similar extent in wild-type and STAT-3-KO mice (29), it is likely that Cyp-D, the target of CsA, is downstream of STAT-3. Boengler et al. also suggest that some protein kinases could be a possible target of STAT-3 within mitochondria (29).

Recently, Bolli and coworkers (36) reported the development of tamoxifen-inducible, cardiomyocyte-specific STAT-3deficient mice. This inducible model represents an attractive model, since it overcomes both the problems of embryonic lethality and the consequences of chronic alterations in gene expression, which may induce adaptive phenotype modifications. These mice are free of alterations in apoptosis, fibrosis, capillary density, cardiac function, and cardiac hypertrophy or dilatation 35 days after the end of the induction treatment with tamoxifen. Therefore, these animals may be of great value to study the role of STAT-3 in the cardioprotection by ischemic PreC and PostC without the confounding effects associated with a chronic STAT-3 deletion. Actually, Bolli and coworkers (36) in this seminal work used this model to study the SWOP (see SWOP; section VIII).

The potential interaction of the RISK, PKG/PKC, and SAFE pathways and their mechanistic relation remain to be defined (137). However, all pathways appear to converge ultimately on the mitochondrion. Whether ischemic PreC and PostC induce a translocation/phosphorylation of STAT-3 into mitochondria is unclear at present. Nevertheless, the targeting of STAT-3 to mitochondria unveils a novel protective approach that is independent of STAT-3 transcriptional activity. The potential beneficial effects of mitochondrial STAT-3 in protecting the myocardium against I/R-mediated damage need further investigation.

4. Pim-1 kinase in the $\mathrm{I} / \mathrm{R}$ scenario and cardioprotection. The proto-oncogene serine/threonine-protein kinase Pim-1 is not yet included in the classical (RISK and SAFE) cardioprotective pathways. This is a kinase that belongs to the family of calmodulin-dependent protein kinases. With Pim-1, a new protein kinase has been identified that increases in the mitochondria after I/R. In fact, the mitochondrial content of Pim-1 is enhanced after I/ $R$ in vitro, and mitochondria isolated from mouse hearts overexpressing Pim-1 are more resistant to $\mathrm{Ca}^{2+}$-induced swelling than mitochondria from wild-type mice (234). Pim-1 operates downstream of Akt, and a feedback mechanism exists involving the two proteins. In fact, Akt is an upstream activator of Pim-1 kinase, and Akt expression and phospho-Akt(Ser473) levels increase in response to Pim-1 overexpression. Similarly, increased levels of phospho-Akt(Ser473) are found in myocardial sections from mice with global genetic deletion of Pim1. Actually, increased levels of phospho-Akt(Ser473), phospho-Akt(Thr308), and total Akt are seen in whole-heart lysates from Pim-1-KO samples (234). Pim-1 inactivation may increase apoptotic activity via increased generation of mitochondrial ROS and mPTP opening, as found in other cellular contexts $(217,234)$. Pim-1 may also act, in part, as a normal upstream regulator of the expression of Bcl-2 (217). Up to now, information on mitochondrial Pim-1 in I/R is scant, and it is unclear whether or not PreC and/or PostC impacts on the mitochondrial contents of Pim-1. Overexpression of Pim-1 was found to protect the myocardium after infarction injury and cardiomyocytes from apoptotic challenge by increasing cell survival signaling (274). While genetic ablation of Pim-1 increased the myocardial infarct size after I/R in vivo (234), its pharmacological inhibition with Pim-1 kinase inhibitor II [2-hydroxy-3-cyano-4-phenyl- 
6-(3-bromo-6-hydroxyphenyl)pyridine] blocked the cardioprotection by PreC (336). We reported that PostC increases the levels of antiapoptotic markers, including Pim-1 and Bcl-2 (274).

5. Bcl-2 family: interaction with mitochondria. The Bcl-2 family consists of three classes of Bcl-2 proteins that can regulate apoptosis. These are (i) inhibitors (e.g., Bcl-2 and Bclxl); (ii) promoters (e.g., Bax and Bak); and (iii) regulators of the antiapoptotic $\mathrm{Bcl}-2$ proteins such as proteins sharing the $\mathrm{BH} 3$ motif (e.g., Bad, Bid, and Bim). These proteins may act as activators or inhibitors of cell death (386). The promoters of apoptosis, Bax and Bak, induce caspase activation via induction of the so-called mitochondrial apoptosis-induced channel (MAC) that is distinct from the MPTP, though also mPTP may be regulated by Bcl-2 family proteins (194). It is thought that Bax interacts with an activator such as Bid (325), which induces a conformational change in Bax, resulting in insertion into the OMM, to form MAC. Release of factors, such as Cyt c and apoptosis-inducing factor (AIF), from the mitochondrial intermembrane space, results in fission of mitochondria into smaller fragments (386). AIF, adenylate kinase, Cyt c, deafness dystonia protein (DDP), Endo G, and DIABLO (also known as Smac) have all been reported to be released from the mitochondrial intermembrane space into the cytosol of cells undergoing apoptosis $(50,386)$. In particular, Cyt c and AIF when released from mitochondria bind to apoptotic peptidase-activating factor 1 (APAF1) $(50,386)$. This will lead to the assembly of the so-called apoptosome (a heptameric protein ring), which will bind and activate caspase 9 . Therefore, once activated, caspase 9 triggers a caspase cascade, which primes the cell for apoptosis (371). A caspaseindependent apoptosis is obtained by the mitochondrial release of other factors, such as AIF and Endo G (50). Smac/ DIABLO released from mitochondria may inhibit the intrinsic inhibitor of apoptosis proteins (IAPs), thereby neutralizing IAPs' caspase inhibitory properties. This may sustain a vicious cycle that could continue to lead to more apoptosis. This vicious cycle could be interrupted by the antiapoptotic $\mathrm{Bcl}$ family proteins by acting on the OMM, thus reducing Cyt $c$ release and subsequently reducing Smac/DIABLO release and decreasing apoptosis (50).

The involvement of Bcl-2 family in the I/R scenario and protection has been extensively studied (119, 194, 217, 274). These studies suggest an important role in reperfusion-induced apoptosis. Both PreC and PostC limit reperfusion injury affecting all form of cell death, including apoptosis (6, $274,355,392)$. Of course, reperfusion must be applied as soon as possible. In fact, both beneficial and deleterious effects of reperfusion as well as protective effects of PostC depend critically on the duration of index ischemia $(125,134,174,226$, $277,329)$. The loss of mitochondrial membrane potential and matrix swelling caused by MPTP opening induce the release of the proapoptotic proteins from the intermembrane space into the cytosol, which ultimately leads to the activation of caspase-mediated apoptosis. Therefore, the Bcl-2 family is of paramount importance in regulating cardioprotection. A direct link between the induction of PreC to protect against I/ R injury and GSK-3 $\beta$ phosphorylation-dependent modulation of mitochondrial Bcl-2 protein levels has been demonstrated (75). Also, PKG activation and enhanced phosphorylation of GSK-3 $\beta$, with a subsequent increase in the Bcl-2/BAX ratio, were implicated in PreC induced by inhibition of phosphodiesterase-5 (73). We were among the first to report a Bcl-2 increase and cleaved caspase- 3 decrease in PostC (274). It has been also reported that PostC may reduce myocardial apoptosis during reperfusion via a JAK-STAT-3-Bcl-2 pathway (355). Further studies focusing on other proteins of the Bcl-2 family may be helpful to define the long-term benefit of PostC against apoptosis $(192,338)$.

6. AMP-activated protein kinase, a metabolic regulator in health and disease. AMPK phosphorylates multiple targets, including several biosynthetic enzymes and cardioprotective elements, such as acetyl-CoA carboxylase, hydroxymethylglutaryl-CoA reductase, glycogen synthase, and endothelial nitric oxide synthase (eNOS) (53). In Figures 6 and 7 are reported some of the multiple targets of activated AMPK.

Usually AMPK is activated when the AMP concentration increases as a result of insufficient ATP production or unmatched energy demand. Therefore, it plays a role of paramount importance in the regulation of mitochondrial function during and after ischemia when the ATP/ADP/AMP undergoes abrupt changes. Although, its main role is to monitor the mitochondrial function and cellular energy status responding to changes in the AMP/ATP ratio, AMPK can also be activated independently of adenine nucleotides, by changes in calcium concentrations as well as by increased production of ROS (16).

AMPK is a heterotrimeric complex that contains a catalytic (alpha) and two regulatory (beta and gamma) subunits. Each

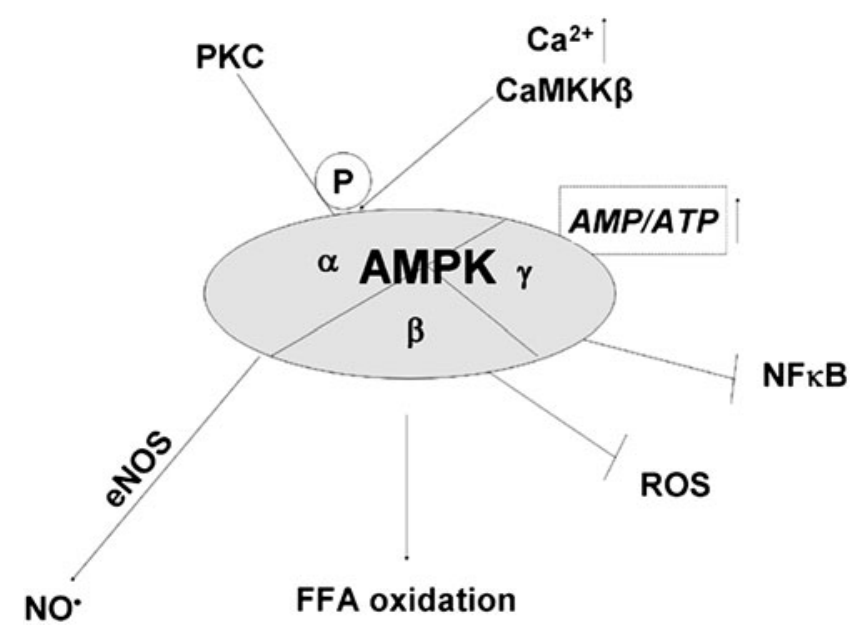

FIG. 6. AMP-activated protein kinase (AMPK) activation. AMPK is formed by $\alpha-, \beta-$, and $\gamma$-subunits. An increased AMP-to-ATP ratio leads to a conformational change in the $\gamma$ subunit, leading to increased phosphorylation and decreased dephosphorylation of AMPK. This activation facilitates the activity of PKC of which AMPK is a substrate. Additional regulation is affected by $\mathrm{Ca}^{2+}$-calmodulin-dependent kinase kinase $\beta(\mathrm{CaMKK} \beta)$, which phosphorylates and activates AMPK in response to increased $\mathrm{Ca}^{2+}$. Activated/phosphorylated AMPK enhances free fatty acid (FFA) oxidation, improves $\mathrm{NO}^{\bullet}$ availability, and may limit ROS production via several different pathways (see also Fig. 7). Once activated, AMPK can download the NF $\kappa$ B-signaling system. For other acronyms, see the list of Abbreviations Used. 


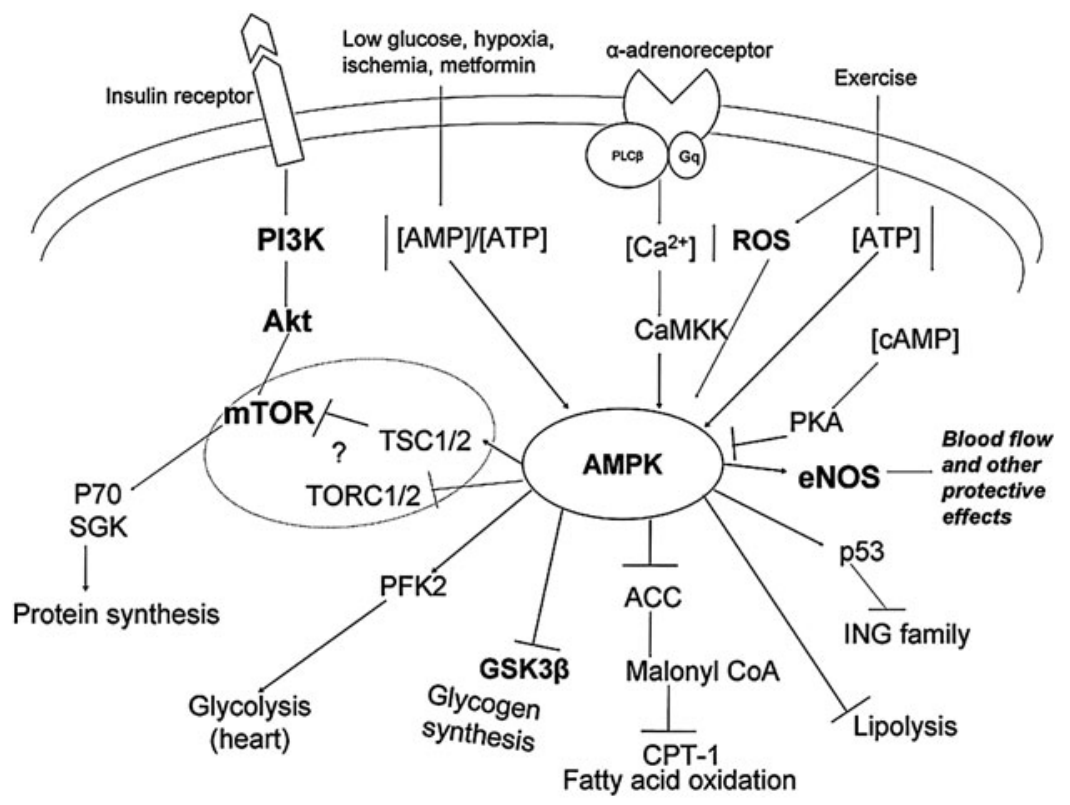

FIG. 7. AMPK signaling in controlling the metabolic state. AMPK regulates protein synthesis, glycolysis, fatty acid oxidation, and lipolysis. AMPK activity is influenced by exercise and hypoxia, likely with the intervention of hormones and metabolites. Here are reported some of the metabolic targets and signaling pathways of AMPK. Activated AMPK has a plethora of targets; for instance, AMPK is a central regulator for cell growth and metabolism via multiple targets, including mTOR (the target of rapamycin), which resides in the two functionally distinct complexes TORC1 and TORC2 (defined by their adaptors Raptor and Rictor, respectively). The two TORCs are affected with unknown modalities. AMPK is also implicated in suppression of initiation and progression of cancers in various tissues via the regulation of the inhibitor of growth (ING) family, an evolutionarily conserved set of proteins. ROS may be activators of AMPK, and activated AMPK may limit ROS production (see Fig. 6 and text). In bold are reported some targets directly involved in cardioprotection (see text). Therefore, AMPK may play a pivotal role in protection and deserves more attention by researchers. PFK2, Phosphofructokinase 2; P70 SGK, glucocorticoid-inducible kinase. For other acronyms, see the list of Abbreviations Used.

subunit has multiple isoforms ( $\alpha 1$ and $2, \beta 1$ and 2 , and $\gamma 1,2$, and 3). Therefore, 12 possible combinations of AMPK holoenzyme are described in murine and human hearts. The catalytic alpha subunit includes both the protein kinase domain and a threonine residue (Thr172) whose phosphorylation by kinases is responsible for AMPK activation. Figure 6 shows the main factors controlling the shift from inactive to active AMPK. It seems that in human hearts, both AMPK $\alpha 1$ and 2 catalytic subunits contribute to the total AMPK activity. On the contrary, in mouse hearts, $\alpha 2$ subunit accounts for about $80 \%$ of total AMPK activity (16). The $\beta$ subunit acts as a scaffold for the other two subunits. It also contains a glycogen-binding domain whose physiological role might be to control glycogen metabolism. AMPK is present in the nucleus and the cytoplasm; however, the mechanisms that regulate the intracellular localization of AMPK are unknown.

It seems that environmental stresses regulate the intracellular localization of AMPK, and upon recovery from heat shock or oxidant exposure, AMPK accumulates in the nuclei. Treatment with an AMPK activator increases the expression of peroxisome proliferator-activated receptor $\gamma$ coactivator (PGC)- $1 \alpha$ and manganese superoxide dismutase (MnSOD) mRNAs, which may inhibit ROS production in mitochondria (204). Since AMPK serves as an energy sensor, it is at the center of control for a large number of metabolic reactions, thereby playing a crucial role in metabolic syndrome (MS), Type-2 diabetes, and other human diseases.
The activation of AMPK induces acceleration of mitochondrial biogenesis in physiological conditions. Conditions leading to changes in AMP concentrations and AMPK activation are directly related to changes in ATP concentrations. Accordingly, ischemia, similarly to mitochondrial respiration inhibitors, activates AMPK within a few minutes (131). Increased ATP demand also leads to AMPK activation, especially when combined with decreased ATP supply, as is the case in contracting muscle during hypoxia. Also, intense exercise, norepinephrine, phenylephrine, isoproterenol, or vasopressin activates heart AMPK (183). In Figure 7 are reported some of the pathways able to activate AMPK and some of the AMPK metabolic targets.

When activated, AMPK aims to restore the cellular energy charge by switching off anabolic ATP-consuming pathways. Although the mechanism remains to be elucidated, AMPK could inhibit (i) cJUN kinase activation; (ii) endoplasmic reticulum stress; and (iii) oxidative stress in several cellular models, including cardiomyocytes (86). Activated AMPK may also inhibit glucose-induced oxidative stress and NADPH oxidase activation in endothelial cells (51). On the other hand, AMPK may switch on catabolic ATP-producing pathways. For these reasons, it may play a role in cell protection. Recently, it has been demonstrated that the AMPKdependent mitochondrial protection of resveratrol against oxidative stress may be associated with the downstream inhibitory phosphorylation of GSK-3 $\beta$ (322). Although the mechanism of resveratrol's cytoprotection involves AMPK 
activation, resveratrol does not directly activate AMPK in vitro (15). It is likely that redox conditions play a role in this mechanism (see section II.C).

\section{B. Important components of mitochondria in the network involved in $I / R$ and cardioprotection}

Kinases may be imported into mitochondria and/or may affect directly and indirectly the function of mitochondrial components (e.g., mPTPs and $\mathrm{mK}_{\mathrm{ATP}}$ channels). Here, we consider more details of these components. However, a challenge is that many mitochondrial channels and transporters have yet to be identified at the molecular level.

1. Mitochondrial permeability transition pore. This pore is a high-conductance megachannel, which, as said, plays a role of paramount importance in the I/R scenario. The modulation of $\mathrm{mPTP}$ is also important physiologically in regulating the calcium homeostasis. The pore modulation and opening will be considered with more detail below (sections III.A, III.B, and III.C); here, we consider the putative components of mPTPs and their interaction. The pore was first described in 1976 by Hunter et al. (167) and is located between the inner and OMMs. When an mPTP is formed, it opens and allows communication between the cytoplasm and the mitochondrial matrix. The molecular identity of the proteins that form this pore is still unknown. It has been suggested that the
mPTP is formed by the VDAC in the OMM, the ANT in the IMM, and Cyp-D in the matrix of mitochondria. When the $\mathrm{mPTP}$ is in the closed state, the matrix protein Cyp-D is detached from IMM, whereas HK II is attached to OMM components of the pore (Fig. 8A). Opening of mPTP (Fig. 8B) appears to be facilitated by the binding of Cyp-D to the IMM in a process regulated by both $\mathrm{Ca}^{2+}$ and inorganic phosphate (Pi) (78). However, experiments with transgenic mice in each of the components of the putative MPTP achieved controversial results. In fact, neither the deletion of the gene nor knockdown of VDAC or ANT prevents mPTP opening in response to $\mathrm{Ca}^{2+}$ overload of mitochondria $(7,8,197,243)$. In fact, experiments with VDAC and ANT KO mice demonstrated substantial permeability transition during $\mathrm{Ca}^{2+}$ stimulation, indicating that VDAC and ANT are not essential for the permeability transition $(7,8,197)$. However, these experiments are not conclusive, and the dispute is not resolved. While in fibroblasts and liver mitochondria of $\mathrm{KO}$ mice Cyp-D Ca ${ }^{2+}$-induced mPTP opening is overdue $(7,12)$, the sensitivity of $\mathrm{mPTP}$ opening in response to adenine nucleotides or oxidative stress is similar in Cyp-D-deficient and wild-type mitochondria, indicating that Cyp-D is important for MPTP opening, but that the permeability transition can occur even in the absence of Cyp-D (12). The possibility that Cyp-D has a role in the regulation of apoptotic proteins in a manner that is independent of the MPTP has been suggested (98).
A Physiological conditions

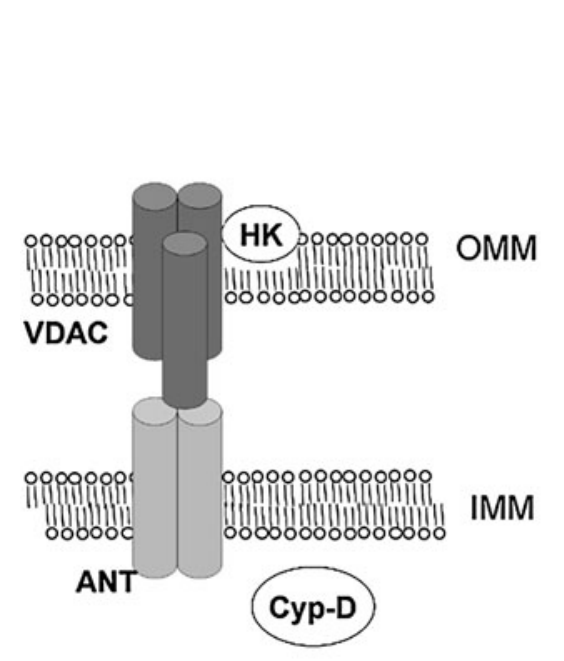

B

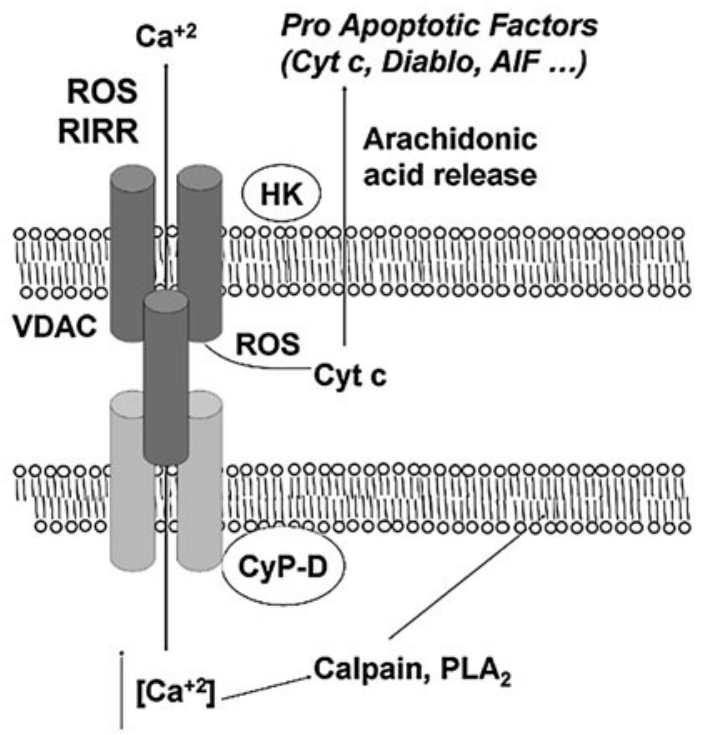

FIG. 8. mPTP composition and regulation. In Figure 1 are reported the factors controlling the mPTP-opening probability. Here are evidenced the putative elements that form the pore. The MPTP is believed to be composed of the ANT in the IMM, the VDAC in the OMM, and Cyp-D in the matrix. (A) In physiological conditions, Cyp-D is detached from IMM, and HK is attached to the OMM. Notably during ischemia, mPTP opening hardly occurs because mPTP is strongly inhibited by acidosis. (B) mPTP typically opens in reperfusion when $\mathrm{Ca}^{2+}$ overload, generation of $\mathrm{ROS}$, and $\mathrm{pH}$ normalization occur. Ca ${ }^{2+}$ overload may stimulate the interaction of Cyp-D with other mPTP components, which triggers permeability transition. A part $\mathrm{Ca}^{2+}$ overload, also inorganic phosphate $(\mathrm{Pi})$, contributes to $\mathrm{mPTP}$ opening through the binding of $\mathrm{Cyp}-\mathrm{D}$ to the inner membrane. Also, HK detachment from mitochondria triggers apoptosis through mPTP opening. In fact, pore opening leads to cell death through the release of proapoptotic factors and via RIRR (ROS-induced ROS release). Other important factors that regulate pore formation are Bax/Bad/Bcl-2 and GSK-3 $\beta$. Cytochrome $\mathrm{c}$ and other mediators are simultaneously released that may play a role in committing the cell to death. These mediators include Smac/Diablo and AIF. For further explanations, see the text and Figure 9. For other acronyms, see the list of Abbreviations Used. 
2. Putative mitochondrial ATP-sensitive potassium channels. Abundant in myocardial sarcolemma, where they were originally discovered (305), ATP-sensitive potassium $\left(\mathrm{K}_{\mathrm{ATP}}\right)$ channels have also been reported to reside within intracellular membranes, including endoplasmic/sarcoplasmic reticulum, nuclei, secretory granules, and mitochondria (78). $\mathrm{K}_{\mathrm{ATP}}$ channels are biosensors that enable high-fidelity readout of metabolic distress signals $(115,347,369)$. It is likely that these channels behave like checkpoints that perform a rheostat-like operation adjusting membrane potentialdependent functions to match energetic demands of the working heart $(124,128,236)$. $\mathrm{K}_{\text {ATP }}$ channel complexes are formed by KCNJ11-encoded Kir6.2 pore subunits coassembled with the regulatory ATP-binding cassette sulfonylurea receptor; channel deficit impairs tolerance to endurance challenge, hemodynamic load, or sympathetic discharge (70, $126,200,207,285,286)$. Both sarcolemmal and putative $\mathrm{mK}_{\mathrm{ATP}}$ channels comprise the protective benefit of ischemic PreC and PostC, $(7,27,82,146,197)$, whereas disruption of the $\mathrm{K}_{\mathrm{ATP}}$ channel blunts this protective response $(197,243)$. Because of the central role of mitochondria in cardioprotection, much attention has been devoted to $\mathrm{mK}_{\mathrm{ATP}}$ channels.

Mitochondrial ATP-sensitive $\mathrm{K}^{+}$channels are located in the IMM, are considered targets of protective signaling pathways, and play a pivotal role in ROS production, mainly $\mathrm{O}_{2}^{-} \cdot$ derived from complex I of the ETC $(27,117,274,310,383)$. Opening of the $\mathrm{mK}_{\mathrm{ATP}}$ channels and subsequent generation of ROS are considered to be a pivotal step in the mechanisms of PreC and PostC $(27,270,382)$. We evidenced that ROS signaling is downstream of $\mathrm{mK}_{\mathrm{ATP}}$ channel opening in isolated rat hearts subjected to $\mathrm{I} / \mathrm{R}$ with an intermittent infusion of $\mathrm{mK}_{\mathrm{ATP}}$ channel opener, diazoxide, or diazoxide plus the ROS scavenger mercaptopropionylglycine (MPG) at the onset of reperfusion. In fact, MPG attenuated diazoxide-induced protection (271).
In the context of cardioprotection, it has been reported that Akt-dependent phosphorylations and subsequent PKG activation lead to $\mathrm{mK}_{\mathrm{ATP}}$ channel activation. Actually, Akt and PKG are a part of the so-called signalosomes (113), which are vesicular, multimolecular signaling complexes, involving the assembly and regulation of multiproteins, which compartmentalize and convey the intracellular signal (296). Whether or not these proteins form a signalosome, they also include eNOS, guanylyl cyclase, and cyclic guanosine monophosphate (cGMP), which activates PKG. Indeed, it has been proposed that activated PKG phosphorylates an unknown protein of the OMM (113). This causes the signal to be transmitted to PKC $\varepsilon$ already bound to the IMM, which in turn causes the $\mathrm{mK}_{\text {ATP }}$ channel opening. $(66-68,113)$.

For the $\mathrm{mK}_{\mathrm{ATP}}$ channel opening, the protective signal is transmitted from the OMM to the IMM. This may be accomplished by a series of intermembrane-signaling steps that includes $\mathrm{PKC} \varepsilon$ activation, so that stimulation of mitochondrial $\mathrm{K}^{+}$influx via the $\mathrm{mK}_{\mathrm{ATP}}$ channels is cardioprotective, and activation of the $\mathrm{mK}_{\mathrm{ATP}}$ channel is mediated by mitochondrial $\mathrm{PKC}_{\varepsilon}$ (Fig. 9). The resulting ROS then activate a second PKC pool, which through another signal transduction pathway causes inhibition of mPTPs (one of the end effectors) and reduction in cell death. In summary, the inhibition of $\mathrm{MPTP}$ opening occurs through intermediary steps involving $P K C \varepsilon$, $\mathrm{mK}_{\mathrm{ATP}}$ channels, $\mathrm{K}^{+}$entry into mitochondria, matrix alkalization, and generation of ROS with a protective signaling role (Figs. 3 and 9) (66-68, 72, 113, 253, 270, 382). Pharmacological opening of $\mathrm{mK}_{\mathrm{ATP}}$ channels by diazoxide contributes to the formation of small amounts of ROS inducing cardioprotection $(74,109,129,142,143)$. Also $\mathrm{NO}^{\bullet}$ donors can activate $\mathrm{mK}_{\text {ATP }}$ channels in rabbit ventricular myocytes and can potentiate the protective effect of the $\mathrm{mK}_{\mathrm{ATP}}$ channel opener diazoxide (310). Besides cGMP/PKG-dependent phosphorylation, $\mathrm{mK}_{\text {ATP }}$ channels can be opened by direct reaction of $\mathrm{NO}^{\bullet}$ and

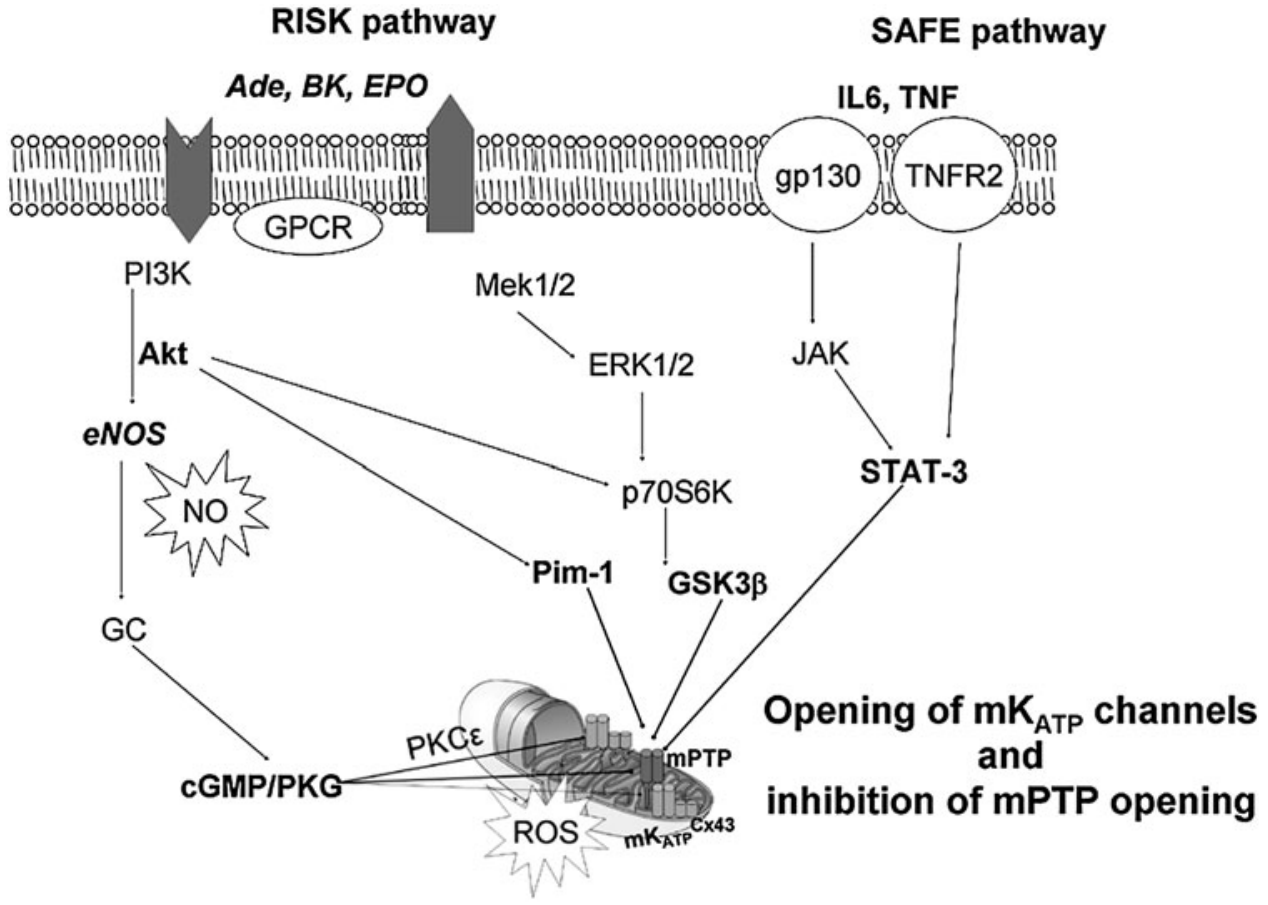

FIG. 9. Signaling pathways in cardioprotection: reperfusion injury salvage kinases (RISKs) and survivoractivating factor enhancement (SAFE). Activation of cell surface receptors (e.g., Ade, BK, erythropoietin [EPO], tumor necrosis factor [TNF], and interleukin-6 [IL6]) in response to a PreC or PostC stimulus may recruit both the RISK and the SAFE pathways. Ligands of membrane receptors initiate cardioprotection by activating a cascade of kinases, leading to $\mathrm{mPTP}$ inhibition, and thus protecting the cell against apoptotic and necrotic death. These two pathways are activated at the time of reperfusion and will crosstalk. See also the text. For other acronyms, see the list of Abbreviations Used. 
derivatives and by the action of $\mathrm{H}_{2} \mathrm{~S}$, leading to S-nitrosylation (SNO) and S-sulfhydration of proteins, respectively [for review see (260)].

It has been suggested that $\mathrm{mK}_{\mathrm{ATP}}$ channels are activated by ROS: $\mathrm{O}_{2}{ }^{-\bullet}$ and $\mathrm{H}_{2} \mathrm{O}_{2}$, but not other peroxides. Yet, certain RNS can activate $\mathrm{mK}_{\text {ATP }}$ via a mechanism involving mitochondrial complex II. Moreover, $\mathrm{mK}_{\mathrm{ATP}}$ channels are inhibited by NADPH. Thus, to sum up, $\mathrm{mK}_{\mathrm{ATP}}$ channels are activated by S-nitrosothiols, nitroxyl, and nitrolinoleate (102, $110,292,389)$. The latter two species also inhibit mitochondrial complex II. Nitroxyl protects cardiomyocytes against IR injury in an $\mathrm{mK}_{\mathrm{ATP}}$-dependent manner. Overall, these results suggest that the $\mathrm{mK}_{\mathrm{ATP}}$ channels are activated by ROS and RNS and inhibited by NADPH. The redox modulation of $\mathrm{mK}_{\mathrm{ATP}}$ channels may be an underlying mechanism for its regulation in the context of PreC and PostC (295).

However, controversy exists on the nature, existence, and opening of $\mathrm{mK}_{\mathrm{ATP}}$ channels, which may also be a deleterious process $(72,74)$. In fact, for instance, $\mathrm{mK}_{\mathrm{ATP}}$ channels are suggested to be involved in angiotensin II-induced redox stress via the depolarization of mitochondrial membrane potential and consequent respiratory dysfunction $(182,216)$. Moreover, the $\mathrm{mK}_{\mathrm{ATP}}$ channel opener diazoxide caused a nitrate tolerance-like phenomenon, whereas the $\mathrm{K}_{\mathrm{ATP}}$ channel inhibitor glibenclamide improved tolerance in nitroglycerintreated animals (71). Finally, diazoxide and/or 5-HD, channel agonist and antagonist, respectively, may also have direct effects on mitochondrial ETC and mitochondrial bioenergetics $(129,130)$. Thus, these drugs lack specificity for the channel, and cautions are needed in this respect.

Hence, it seems that on the one hand, $\mathrm{mK}_{\mathrm{ATP}}$ channel opening induced by PKC activation stimulates ROS production, whereas on the other hand are ROS generated in the cytosol that stimulate $\mathrm{mK}_{\text {ATP }}$ channel opening. A simple model in which the first mechanism may be protective while the second would be detrimental could be suggested. However, PKC activation leading to the opening of $\mathrm{mK}_{\mathrm{ATP}}$ channels has been challenged by the Halestrap group: they demonstrated that PreC inhibits opening of the MPTPs in situ by an indirect mechanism probably involving decreased ROS production and $\mathrm{Ca}^{2+}$ overload at reperfusion $(74,128)$. Therefore, based on these observations, one can speculate that $\mathrm{mK}_{\mathrm{ATP}}$ channel activation by PKC is not required to induce protection. Nevertheless, several studies have shown that redox-dependent opening of the channel is a protective mechanism [for reviews see $(66,67,295)]$.

Clearly, further work in this area, including the molecular identification of the $\mathrm{mK}_{\mathrm{ATP}}$ channel itself and the redox-sensitive residues within it, will facilitate a better understanding of the role that regulation of the channel plays in events such as cardioprotection.

3. Mitochondrial Cx43. A component that has been associated with the $\mathrm{mK}_{\mathrm{ATP}}$ channel structure and function is mitochondrial Cx43 (mCx43). Indeed, within cardiomyocytes, Cx43 is mainly localized in the cellular membrane at gap junctions. Cx43 is formed by four transmembrane domains, two extracellular and one intracellular loop, as well as cytosolic amino- and carboxytermini. Cx43 is the target of different kinases, among them protein kinase A (PKA), PKC, and MAPKs, which phosphorylate Cx43 sites mainly located within the carboxyterminus [for a review, see (331)].
However, Cx43 is also present in other organelle membranes, including the IMM of a subset of cardiomyocyte mitochondria, that is, subsarcolemmal mitochondria $(26,32$, 222), where $\mathrm{C} x 43$ regulates mitochondrial $\mathrm{K}^{+}$fluxes (232, 304). Cx43 presence has not been observed at the inner membrane of cardiomyocyte interfibrillar mitochondria (32). Whether the half-life of $\mathrm{mCx} 43$ is similar to that of gap-junctional Cx43 (about $90 \mathrm{~min}$ ) is unknown at present. The pathway by which $\mathrm{Cx} 43$ is imported into subsarcolemmal mitochondria involves Hsp90 and Tom $20(32,274)$. Since the inhibitor of Hsp90, geldanamycin, reduces the $\mathrm{mCx} 43$ content in hearts perfused under normoxic conditions, it is possible that $\mathrm{C} \times 43$ is continuously imported into mitochondria.

Interestingly, $\mathrm{mCx} 43$ coimmunoprecipitates with GSK-3 $\beta$ (32). mCx43 has also been implicated in ROS signaling, though its role is not completely defined $(27,28,32)$. It has been suggested that $\mathrm{C} x 43$ may form the pore of $\mathrm{mK}_{\mathrm{ATP}}$ channels (304). In fact, mitochondrial $\mathrm{PKC} \varepsilon$ phosphorylates $\mathrm{C} \times 43$, and the PKC $\varepsilon$-mediated activation of the $\mathrm{mK}_{\mathrm{ATP}}$ channel requires $C x 43$ (66). As said above, opening of the $\mathrm{mK}_{\mathrm{ATP}}$ channel contributes to cardioprotection via the formation of small amounts of ROS, which function as trigger molecules of PreC and PostC. However, since the open probability of the channel is not dependent on $\mathrm{Cx} 43$ under basal conditions, it is unlikely that $\mathrm{C} \times 43$ represents the pore-forming unit of the $\mathrm{mK}_{\text {ATP }}$ channel (32).

$\mathrm{mCx} 43$ has been described to be essential for PreC protection $(142,143,301,318)$, but a study $(146)$ in mice heterozygous for $\mathrm{Cx} 43\left(\mathrm{C} \times 43^{+/-}\right)$indicates that it does not play a significant role in PostC protection. Actually, Cx43 is a target of several protein kinases, and $\mathrm{mCx} 43$ is highly phosphorylated under physiological conditions (357); it seems that in the IMM of subsarcolemmal mitochondria, the phosphorylated portion of Cx43 increases with ischemia (32) and decreases with PostC (274). Since a decrease of the $\mathrm{mCx} 43$ content is sufficient to abolish the cardioprotection by diazoxide PreC, that is, reduces ROS formation $(142,143)$, one can speculate that $\mathrm{mCx} 43$ reduction in PostC may be one of the mechanisms to reduce excessive ROS production in the reperfusion phase. Recently, it has been suggested that genetic ablation or pharmacological inhibition of $\mathrm{mCx} 43$ confers resistance to $\mathrm{mK}_{\mathrm{ATP}}$ channel opening in response to diazoxide in patchclamped mitoplasts (mitochondria devoid of the OMM) (304). However, the open probability of the $\mathrm{mK}_{\mathrm{ATP}}$ channel was not affected under baseline conditions; thus, it is likely that $\mathrm{mCx} 43$ regulates this channel activity rather than constituting the pore-forming unit of the $\mathrm{mK}_{\mathrm{ATP}}$ channel (304). Cardiac cells from heterozygous Cx43-deficient mice do not form sufficient amounts of ROS when stimulated by diazoxide and accordingly, have no cell death reduction after I/ $\mathrm{R}$ in vivo. Ischemic PreC increases the amount of $\mathrm{mCx} 43$ after short intermittent cycles of PreC ischemia in rat hearts in vitro and after infarcting ischemia in pig hearts in vivo. In this latter species, I/R is associated with increased dephosphorylation of $\mathrm{mCx} 43$, and ischemic PreC preserves Cx43 phosphorylation at Ser368 $(74,129,143)$. However, enhanced mCx43 content after PreC seems not a prerequisite for cardioprotection. It is rather the decrease of $C \times 43$ below a certain threshold level that should be avoided to allow the ROS-triggered protection of the heart by PreC.

The role of $\mathrm{mCx} 43$ in ischemic PostC has not been clearly characterized up to now. We demonstrated that decreased 
amounts of total Cx43 in mitochondria isolated from postconditioned (five cycles of 10-s ischemia and reperfusion each) rat hearts underwent 30-min ischemia and 120-min reperfusion in an ex vivo model (274). The reduced amounts of total Cx43 were paralleled by reduced amounts of Cx43 phosphorylated at the epitope Ser368. Heusch et al. (146) have studied the $\mathrm{C} \times 43$ content in mitochondria isolated from mouse hearts subjected to $30-\mathrm{min}$ ischemia and $10-\mathrm{min}$ reperfusion without and with PostC (three cycles of 10-s ischemia and reperfusion each) in vivo. In this model, PostC increased both the levels of total Cx43 and of Cx43 phosphorylated at Ser368. However, ischemic PostC reduced the infarct size in heterozygous Cx43-deficient hearts to a similar extent as in wild-type hearts.

Therefore, physiological amounts of $\mathrm{mCx} 43$ are important for effective cardioprotection by ischemic and pharmacological PreC. mCx43 plays an important role in PreC likely via modulation of the opening of $\mathrm{mK}_{\text {ATP }}$ channels and subsequent ROS signaling. The role in PostC is less clear. We suggest that an initial involvement and a subsequent decrease of the amounts of $\mathrm{mCx} 43$ may play a role in PostC, underpinning the initial triggering role of ROS signaling and the subsequent reduction of redox stress. Alternatively, changes in the amount of total or phos- phorylated $\mathrm{C} \times 43$ may be an epiphenomenon associated with ischemic PostC.

4. Mitochondrial uncoupling proteins. Other mitochondrial components associated with ROS production are the uncoupling proteins (UCPs). Under physiological conditions, mitochondrial oxygen consumption $\left(\mathrm{VO}_{2}\right)$ is coupled to ATP synthesis. Reducing equivalents, resulting from energy substrate oxidation, deliver electrons to the mitochondrial ETC. The energy resulting from electron transfer to oxygen atom is used to generate an electrochemical gradient by pumping protons from the mitochondrial matrix into the intermembrane space. Under physiological conditions, the protons reenter the matrix via $\mathrm{F}_{0} \mathrm{~F}_{1}$-ATPase, which uses the energy to regenerate ATP from ADP (Fig. 10). In addition, a small proportion of $\mathrm{H}^{+}$can bypass $\mathrm{F}_{0} \mathrm{~F}_{1}$-ATPase, so that $\mathrm{VO}_{2}$ is not strictly coupled to ATP synthesis. In the $1970 \mathrm{~s}, \mathrm{H}^{+}$traslocases were identified in the IMM of brown adipose tissue and named UCPs, which are present in the mitochondria of different tissues and have different homologs. There are four UCP variants believed to induce inward $\mathrm{H}^{+}$leak in energized mitochondria. Their main role is to direct the mitochondria to produce heat rather than ATP, that is, there is an $\mathrm{H}^{+}$influx into the mitochondrial matrix without phosphorylation of

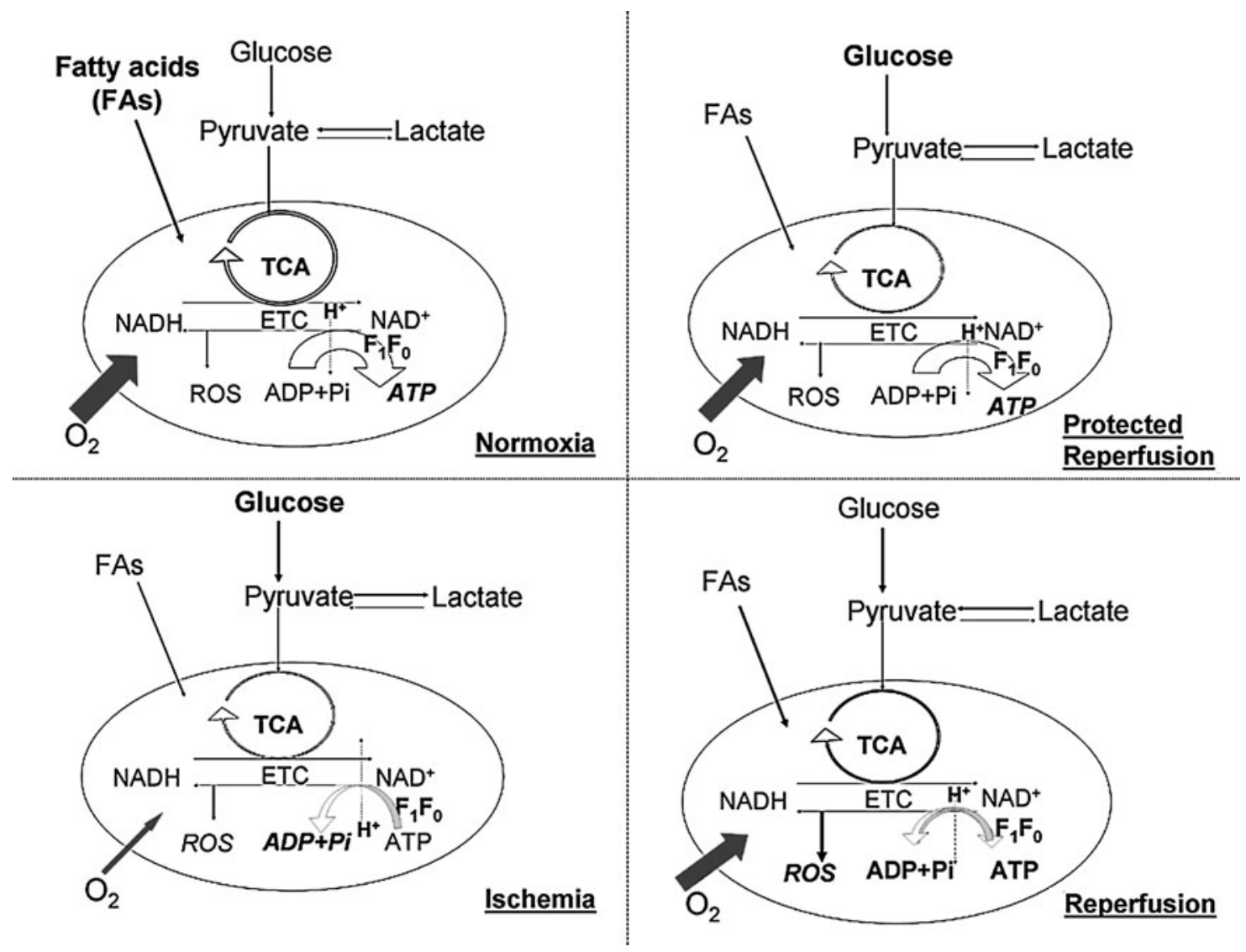

FIG. 10. $F_{0} F_{1}-A T P a s e$ and ETC function in ischemia and reperfusion with and without protection. $F_{0} F_{1}-A T P a s e$ uses the energy of the proton-electrochemical gradient $\left(\Delta \mathrm{H}^{+}\right)$across the IMM to regenerate ATP from ADP. During ischemia, it can work in a reverse mode and consumes ATP. During reperfusion, $\mathrm{F}_{0} \mathrm{~F}_{1}-\mathrm{ATPase}$ and ETC restart to produce ATP and ROS in a variable quantity, whether the heart is protected or not. In nonprotected reperfusion when mPTP is opened, $\mathrm{F}_{0} \mathrm{~F}_{1}-\mathrm{ATPase}$ can work in a reverse mode. The thickness of arrows indicates the prevalent fuel utilized (in bold) and the molecules produced (in bold-italic) by mitochondria. TCA, tricarboxylic acid cycle. For other acronyms, see the list of Abbreviations Used. 
ADP. As such, UCPs are important metabolic regulators in permitting fat oxidation and in attenuating free-radical production. Since ROS production increases with increasing membrane potential, UCP-mediated uncoupling has been proposed to play a role in regulating mitochondrial ROS production and may represent a mechanism by which mitochondria protect themselves from oxidative damage (40, 317). Among ROS-derived lipid peroxidation products, 4hydroxynonenal (HNE) is considered an important mediator of free-radical damage (101). It has been suggested that cytotoxic HNE is also able to act as a signaling agent, inducing mitochondrial uncoupling via the UCP1, UCP2, UCP3, and ANT (94). The mechanism of induction of proton leak by HNE is unclear. It has been suggested that HNE may induce covalent modification and conformational changes in the UCPs that allow the passage of protons back into the mitochondrial matrix (95). Both UCP2 and 3 are expressed in the heart, but their role is unclear. Although controversy exists on the cardioprotective role of uncoupling, mild uncoupling secondary to activation of UCPs has been described to confer cardioprotection under several conditions, including myocardial reperfusion, likely by decreasing ROS production (157). For instance, both $\mathrm{ONOO}^{-}$and electrophilic lipids can activate cardioprotective mitochondrial mild uncoupling (41, 229) Moreover, conditions occurring during PreC, such as elevated $\mathrm{NO}^{\bullet}$, transient ROS generation, acidic $\mathrm{Ph}$, and the activation of both mitochondrial phospholipase A2 and lipoxygenases $(63,237,376)$, could favor nitroalkene generation from the polyunsaturated fatty acids in mitochondrial membranes. Nitroalkenes formed in mitochondria during ischemic PreC nitroalkylated ANT and UCP2, leading to mild uncoupling and cardioprotection against I/ R injury (240). Yet mitochondrial uncoupling contributes to reduced ROS formation (306). Intriguingly, it has been suggested that transient complex I inhibition during reperfusion is cardioprotective via attenuated ROS production (324). Moreover, conditions leading to elevated $\mathrm{NO}^{\bullet}$, transient $\mathrm{ROS}$ generation, acidic $\mathrm{pH}$, and thus to mitochondrial uncoupling may occur also in PostC (see below). Nevertheless, experimental evidence supporting the involvement of uncoupling in PostC protection has yet to be provided.

\section{ROS/RNS: from mitochondria to activation of kinases of the network}

ROS and RNS such as $\mathrm{O}_{2}{ }^{-\bullet}, \mathrm{H}_{2} \mathrm{O}_{2}$, and $\mathrm{ONOO}^{-}$are small and highly reactive molecules having both physiological (ROS/RNS signaling) and pathological effects (ROS/RNS stress) (Fig. 5). In several systems, various pathways, particularly those involving MAPKs (e.g., JNK, ERK, and p38 MAPK) and PKCs, are modulated by ROS and RNS. It is now clear that normal levels of cellular ROS and RNS play important roles in cell-signaling pathways and are vital for physiological functions. For instance, $\mathrm{H}_{2} \mathrm{O}_{2}$ of mitochondrial origin are of paramount importance in metabolic coronary vasodilatation (307).

ROS are generated in different cellular compartments and by several enzymes, including NADPH oxidases at the plasma membrane (211) and cytosolic xanthine oxidases (111). Although ROS are produced by several extracellular and intracellular processes, in cardiomyocytes, mitochondria represent the most relevant site for $\operatorname{ROS}$ formation $(91,238$,
363-365). It has been suggested that ROS-mediated signals (mainly by $\mathrm{H}_{2} \mathrm{O}_{2}$ ) arising within mitochondria can also generate a retrograde response that is conveyed to the nucleus, causing the upregulation of nuclear genes encoding mitochondrial proteins and leading to the induction of mitochondrial biogenesis (48). Also, $\mathrm{NO}^{\bullet}$ is known to induce the production of $\mathrm{O}_{2}{ }^{-\bullet}$ and $\mathrm{H}_{2} \mathrm{O}_{2}$ by mitochondria, and may trigger redox signaling $(46,48,240,241,294,300)$ (see also below).

Within mitochondria, the largest amount of oxygen is reduced to water at respiratory complex IV. Indeed, even under physiological conditions, a minor fraction of oxygen $(<0.1 \%)$ is transformed into $\mathrm{O}_{2}{ }^{-\bullet}$ at the level of complexes I and III; SOD then rapidly converts $\mathrm{O}_{2}{ }^{-\bullet}$ to $\mathrm{H}_{2} \mathrm{O}_{2}$ that is freely diffusible through membranes (110). The production of $\mathrm{H}_{2} \mathrm{O}_{2}$ can increase during myocardial challenging, such as during increased cardiac work load (307). The further reduction of $\mathrm{H}_{2} \mathrm{O}_{2}$ to $\mathrm{OH}^{\bullet}$ in the presence of transition metals, such as $\mathrm{Fe}^{2+}$ or $\mathrm{Cu}^{2+}$, represents a dangerous step, because an increase in toxicity can occur. In fact, no enzyme is available for the removal of $\mathrm{OH}^{\bullet}$ that can only be scavenged by antioxidants with the formation of less-dangerous radical species.

The mitochondrial formation of ROS might be modulated by $\mathrm{NO}^{\bullet}(46,294,300)$, which reversibly inhibits cytochrome oxidase $(20,363)$. This inhibition can be transformed into irreversible alterations of respiratory chain when $\mathrm{NO}^{\bullet}$ reacting with $\mathrm{O}_{2}^{-}$generates a great amount of $\mathrm{ONOO}^{-}$, an $\mathrm{RNS}$ that can produce irreversible nitration of proteins, including proteins from the oxidative phosphorylation system $(18,220,362$, 391). Under certain conditions, such as scarcity of substrate and/or cofactors, NOS can become uncoupled, resulting in the generation of $\mathrm{O}_{2}^{-\bullet}$ and/or $\mathrm{OH}^{\bullet}$, instead of $\mathrm{NO}^{\bullet}(18,379)$. Recent evidence suggested neuronal NOS (nNOS) as a protein completely incorporated to the IMM in a physiological context. In particular, evidence on the presence of an nNOS $\alpha$ variant in heart mitochondria was provided by Kanai et al. (188), with the electrochemical determination of the $\mathrm{Ca}^{2+}$. induced $\mathrm{NO}^{\bullet}$ release from a single mouse heart mitochondrion, a process that was absent in $\mathrm{nNOS}^{-1-} \mathrm{KO}$ mice. Mitochondrial NOS uncoupling has also been postulated (106), and it could play a role in I/R injury, though this remains to be demonstrated.

Apart from the ETC, ROS can also be produced in the intermembrane space by the apoptosis promoter p66Shc, by monoamine oxidases (MAOs), and by AIF located in the OMM (Fig. 5). The p66Shc oxidizes reduced Cyt c, thus inducing the partial reduction of oxygen to peroxide. While under physiological conditions p66Shc is present in the cytosol, under stress conditions, it can be phosphorylated by PKC $\beta$ and translocated into the mitochondrial intermembrane space. The monoamine catabolism generates aldehydes, ammonia, and $\mathrm{H}_{2} \mathrm{O}_{2}$. In fact, MAOs transferring electrons from amine compounds to oxygen generate $\mathrm{H}_{2} \mathrm{O}_{2}$. AIF comprises NADH oxidase activity and can generate superoxide anion $(82,91,114,256)$.

Besides being a relevant site for ROS formation, mitochondrial function and structure are profoundly altered by oxidative stress (82), especially when mPTPs were undergoing prolonged opening. In fact, mPTPs play a central role in the so-called ROS-induced ROS release (RIRR) (396-398); excessive ROS facilitate $\mathrm{mPTP}$ opening, which in turn favors ROS formation by inhibiting the respiratory chain because of 
the mPTP-induced loss of Cyt $\mathrm{c}$ and pyridine nucleotides (82, 127). This vicious cycle is likely to be established at the onset of a rapid reperfusion when a large increase in ROS formation occurs along with $\mathrm{pH}$ recovery and $\mathrm{Ca}^{2+}$ overload, thus inducing injury amplification, as mentioned above and discussed below (Fig. 1).

ROS are important regulators of PKC by reacting with thiol groups associated with the zinc-finger region of the molecule (199). RNS-dependent activation of PKC, possibly via a redoxsensible SNO process, has been also suggested, a process also occurring within mitochondria $(260,288,341)$. A recent hypothesis considers that $\mathrm{H}_{2} \mathrm{O}_{2}$ and aminoimidazole carboxamide ribonucleotide (AICAR) treatments phosphorylate and activate AMPK (99). The $\mathrm{H}_{2} \mathrm{O}_{2}$-mediated activation of AMPK is likely mediated via the ROS-induced decrease in ATP levels. Endogenously produced ROS within skeletal muscle cells are important for the maintenance of PGC- $1 \alpha$ mRNA expression. The PGC- 1 family of regulated coactivators, consisting PGC$1 \alpha$ and PGC- $1 \beta$ as well as PGC- $1 \alpha$-related coactivator, may control mitochondrial respiratory function and biogenesis. A potential link between ROS and the induction of mitochondrial biogenesis has been established. In fact, the PGC-1 $\alpha$ gene expression is regulated by ROS, and PGC- $1 \alpha$ plays a central role in regulating the mitochondrial content within cells (312, 335). Also, kinases are involved in mitochondrial biogenesis, and the most important of these include calcium-/calmodulin-dependent protein kinase IV (378), AMPK (177), and p38 MAP kinase. At least two separate mechanisms are involved in increasing PGC- $1 \alpha$ mRNA expression in response to AICAR. One involves AMPK activation, leading to increases in PGC- $1 \alpha$ promoter activity and mRNA expression. The other is mediated by a reduction in cellular ROS levels, which leads to a reduction in the level of PGC- $1 \alpha$ mRNA expression. Therefore, it is likely that the effect of AICAR on PGC-1 $\alpha$ gene expression is a balance between the positive effects of AMPK activation on promoter activity and its negative effect of ROS, which may enhance mRNA decay (176).

Also, AMPK-mediated protection could be redox-sensitive: both $\mathrm{H}_{2} \mathrm{O}_{2}$ and increased production of radicals activate AMPK (99), possibly by oxidation of two cysteine residues in the alpha subunit of AMPK (395). For instance, it has been demonstrated that metformin, a drug used to treat type II diabetes, inhibits complex I of the respiratory chain to generate mitochondrial $\mathrm{O}_{2}{ }^{-\bullet}$, and then $\mathrm{ONOO}^{-}$, which leads to AMPK activation via a c-Src- and PI3K-dependent pathway (400). Subtoxic concentrations of $\mathrm{ONOO}^{-}$generated by metformin activate AMPK to precondition the cells, which subsequently reduces the excessive oxidant stress triggered by hyperglycemia, so that a cross-talk exists between redox conditions, activity of kinases, and factors promoting mitochondrial biogenesis, which, in turn, may regulate ROS and RNS production in acute and chronic conditions.

Therefore, the key message here is that the signal transduction pathways converge on mitochondria, and are enhanced by ROS/RNS. That is, they act as secondary messengers to control a variety of physiological responses (redox signaling), such as the regulation of vascular smooth muscle tone, the regulation of mitochondrial respiration, and the progression/enhancement of signaling pathways. The latter involves kinases, such as PKC, MAPK, and AMPK, as well as some transcription factors. Yet, sources of superoxide such as the mitochondrial ETC, in the RIRR mechanisms, and xanthine oxidase are not tightly regulated and may become increasingly relevant in the pathology (redox stress) of $I / R$ injury.

\section{Role of Mitochondria in Acute I/R Injury}

As said, under physiological conditions, mitochondria are the principal organelles that generate ATP. This is particularly true for the cardiomyocytes, which are among the richest cells in mitochondria. These organelles take also a part in a series of metabolic processes and signaling pathways, including cell division, and regulate cell death and life. Importantly, during the normal heart perfusion, mitochondria consume large amounts of $\mathrm{O}_{2}$ to produce energy with a minimal loss of electrons to generate $\mathrm{O}_{2}{ }^{-\bullet}$. They contribute to a balanced generation and scavenging of ROS and are involved in cellular ion homeostasis, including $\mathrm{Ca}^{2+}$ homeostasis. The regulation of these processes is crucial for cell function, life, and death.

Deviation from physiological conditions may be, for instance, increased mitochondrial $\mathrm{Ca}^{2+}$ concentration (calcium overload) and increased production of ROS (oxidative stress). Mitochondrial $\mathrm{Ca}^{2+}$ overload and oxidative stress can culminate in prolonged $\mathrm{mPTP}$ opening and reduced synthesis of ATP. These processes can be a part of the pathophysiology of various cardiovascular diseases, including stroke and acute myocardial I/R.

During ischemia, the lack of $\mathrm{O}_{2}$ inhibits the flow of electrons, and the use of myocardial ATP becomes inefficient. The proton-translocating $\mathrm{F}_{0} \mathrm{~F}_{1}$-ATP synthase, which normally produces ATP, goes into a reverse mode, so that it becomes an $\mathrm{F}_{0} \mathrm{~F}_{1}$-ATPase and consumes ATP to pump protons from the matrix into the intermembrane space (Fig. 10). Depending on the species and ischemic conditions, $35 \%-80 \%$ of ATP is consumed by $\mathrm{F}_{0} \mathrm{~F}_{1}$-ATPase during ischemia (124), and the ATP is used to maintain the mitochondrial membrane potential $(\Delta \psi \mathrm{m})$, instead of supporting contractile function to fuel ion pumps to maintain the cellular homeostasis of ions such as that occurs during normal perfusion (236). This alters ATP/AMP ratios and AMPK activation (see above). Due to decreasing levels of ATP during ischemia, $\mathrm{Na}^{+} / \mathrm{K}^{+}$ATPase is inhibited, and intracellular acidification (induced by the production of lactate and the hydrolysis of ATP) activates the $\mathrm{Na}^{+} / \mathrm{H}^{+}$exchanger (NHE, i.e., the cell tries to restore the intracellular $\mathrm{pH})$, and the resulting increase in intracellular $\mathrm{Na}^{+}$activates in a reverse mode the $\mathrm{Na}^{+} / \mathrm{Ca}^{2+}$ exchanger (NCE), leading to $\mathrm{Ca}^{2+}$ overload. High concentration of cytosolic $\mathrm{Ca}^{2+}$ may contribute to cell damage through the activation of degrading enzymes such as nucleases, phospholipases, and proteases, leading to the destruction of the integrity of the membrane and cell death if the ischemic period is long enough. The mitochondria become de-energized at least in part, by inhibiting the $\mathrm{Ca}^{2+}$ uniporter, the major route of entry of $\mathrm{Ca}^{2+}$ in mitochondria. However, during prolonged cardiac ischemia, $\mathrm{Ca}^{2+}$ accumulation in mitochondria can occur through the reversal of the NCE $(127,128)$.

At the reperfusion, intracellular and mitochondrial events such as inadequate resynthesis of ATP, oxidative stress from ROS, low production of $\mathrm{NO}^{*}, \mathrm{Ca}^{2+}$ overload, and loss of membrane phospholipids contribute to reperfusion injury (207, 208, 285) (Fig. 1). Increased concentrations of ATP via recovered oxidative phosphorylation may contribute to the 
restoration of cellular homeostasis of ions; however, a higher content of ATP paradoxically contributes to reperfusion injury (286). In fact, in rapidly re-energized cells, $\mathrm{Ca}^{2+}$ oscillations between the sarcoplasmic reticulum and the cytosol contribute to the hypercontraction of cardiomyocytes, disruption of the membrane, and consequent band necrosis (285287). In particular, it was reported that recovery of $\mathrm{pH}, \mathrm{Ca}^{2+}$ overload, and oxidative stress can cause the sudden opening of the large conductance pore, $\mathrm{mPTP}$, which contributes strongly to cardiomyocyte hypercontraction, apoptosis, and necrosis $(27,78,81,146,305)$.

\section{A. MPTP opening in acute $I / R$}

Of note, MPTP opening can be temporary, intermediate, or prolonged, depending on the complex balance between inducers and antagonists $(282,283)$. The transient opening of $\mathrm{mPTP}$ is able to generate reversible cellular changes, so that this temporary opening has been suggested to be involved in physiological processes and cardioprotection (185). In reality, the transient pore opening is involved in physiological events such as the reversible intracellular trafficking of $\mathrm{NAD}^{+}(80)$, the rapid discharge of excessive intramitochondrial $\mathrm{Ca}^{2+}(21,171,172)$, and the transient formation of ROS (374) (see also section III.C).

Prolonged opening of the $\mathrm{mPTP}$ represents a key event leading to cell death in the pathophysiology of I/R injury (337, 377). In fact, the opening of $\mathrm{mPTP}$ is central to the oxidative damage associated with reperfusion. Many studies have highlighted an important contribution of mPTP opening and cell death that correlated with the release of Cyt c after Bax and enhanced levels of ROS. When an MPTP is formed, mitochondria are uncoupled, and ATP production is blocked, and the matrix swells and can cause the OMM rupture (Figs. 1 and 8 ) [for reviews see $(78,81,82)]$.

It is important to note that the probability of mPTP opening of mitochondria de-energized is drastically reduced below $\mathrm{pH}$ 7.4 , a condition that occurs during prolonged ischemia $(64,62$, $112,274)$. Low $\mathrm{pH}$ also favors the activation of both mitochondrial NHE and NCE, and consequently reduces the uptake and extrusion of $\mathrm{Ca}^{2+}$ from the mitochondrial matrix (175). Still, the low $\mathrm{pH}$ can also activate UCPs. These would uncouple ATP synthesis from oxygen consumption (96). Whether UCPs play a deleterious or protective role in tolerance against ischemia is controversial $(24,157)$. Low $\mathrm{pH}$ can also inhibit glycolysis and the production of pyruvate, resulting in a lower production of acetyl-CoA for the tricarboxylic acid cycle. Finally, this will result in a slower feeding of the respiratory chain complexes. Therefore, low $\mathrm{pH}$ prevents $\mathrm{mPTP}$ opening, mainly in ischemia.

Upon reperfusion, very different conditions are established depending on whether or not $\Delta \psi \mathrm{m}$ rapidly recovers. In the case of energized respiring mitochondria, a low $\mathrm{pH}$ stimulates the uptake of $\mathrm{Pi}$, raising its intramitochondrial content that acts as a potent inducer of mPTP opening. For example, in brain-energized mitochondria, low $\mathrm{pH}$ has been reported for mPTP opening (202). In contrast, in the presence of mitochondrial membrane depolarization, prolonged opening of $\mathrm{mPTP}$ takes place when a rapid normalization of tissue $\mathrm{pH}$ occurs. This last condition (i.e., membrane depolarization and rapid normalization of $\mathrm{pH}$ ) is the most common scenario during abrupt reperfusion. The $\mathrm{mPTP}$ opening is facilitated by the presence of elevated levels of $\mathrm{Ca}^{2+}, \mathrm{Pi}$, and ROS and by the presence of low levels of $\mathrm{NO}^{\bullet}$. In fact, when reperfusion occurs, metabolic and biochemical changes take place in the postischemic cardiomyocytes, including a rapid restoration of normal $\mathrm{pH}$ by membrane exchangers (especially NHE), generation of ROS (including RIRR), an intracellular $\mathrm{Ca}^{2+}$ overload, resulting in mitochondrial dysfunction and possibly cell death $(78,79,286,398)$. In addition to its direct action on mitochondria, the opening effect of $\mathrm{Ca}^{2+}$ overload is also due to indirect aspects such as swelling, band necrosis, and enzyme activation. For example, activation of phospholipase A2 (319), which facilitates the release of arachidonic acid from phospholipids, and activation of calpain proteinases are both $\mathrm{Ca}^{2+}$ dependent (325). These enzymes facilitate further mPTP opening (Fig. 8) (175).

1. Consequences of prolonged mPTP opening. Transient pore opening may be protective, and transient/intermediate opening may lead to apoptosis. Prolonged pore opening, on the other hand, is followed by profound changes in cellular bioenergetics that are considered irreversible. Pore opening results in an increase in mitochondrial permeability to ions and solutes with molecular weights up to $1500 \mathrm{Da}$, matrix swelling, loss of electrochemical gradients, and $\mathrm{F}_{0} \mathrm{~F}_{1}$-ATPase hydrolyses of ATP, leading to an inevitable cell death $(81,121,122,127,128)$. Actually, the consequence of prolonged mPTP opening is the collapse of mitochondrial membrane potential. This is quickly followed by $\mathrm{NAD}^{+}$and ATP depletion, mitochondrial release of accumulated $\mathrm{Ca}^{2+}$, matrix swelling, and rupture of the OMM. The consequence may be loss of pyridine nucleotides and release of proapoptotic factors as Cyt c, which triggers apoptosis and thus inhibits the flow of electrons through the ETC $(21,78,81,83,128,146,398)$.

Many have suggested that the formation of long-term $\mathrm{mPTP}$ is the event that leads to irreversible changes in cellular function and death (e.g., 70 and 121). Di Lisa et al. (83) were among the first to observe that the addition of $\mathrm{Ca}^{2+}$ causes swelling of mitochondria and profound decreases in $\mathrm{NAD}^{+}$content. Global ischemia in isolated, perfused rat hearts increased by the $30 \%$ depletion of tissue $\mathrm{NAD}^{+}$, whereas reperfusion aggravated the loss of tissue $\mathrm{NAD}^{+}$, which remains $\sim 20 \%$ of preischemic levels. Mitochondrial $\mathrm{NAD}^{+}$levels after $\mathrm{I} / \mathrm{R}$ were only about $10 \%$ of the preischemic level (83). In particular, these changes in tissue and mitochondrial $\mathrm{NAD}^{+}$levels after I/R were largely prevented by the mPTP desensitizer, CsA.

\section{B. Prevention of prolonged MPTP opening}

All these opening factors are countered by physiological mPTP antagonists, such as adenine nucleotides (particularly $\mathrm{ADP}$ ), high concentrations of protons (i.e., $\mathrm{pH}$ below 7.4), increased mitochondrial membrane potential, and magnesium ions, as well as by physiological levels of $\mathrm{NO}^{\bullet}(78,372)$. The pore quickly closed if $\mathrm{Ca}^{2+}$ is chelated $(21,78)$. The events promoting $\mathrm{mPTPs}$ are prevented by some drugs (see section $\mathrm{X}$ ), including CsA, which is an mPTP desensitizer at $\mathrm{nM}$ concentrations (12). Paradoxically, it was suggested that under some conditions, Pi may be an inhibitor of mPTP. In the absence of $\mathrm{Pi}$, desensitizing effects of CsA or Cyp-D ablation are no longer present (12).

In summary, the prolonged opening of $\mathrm{MPTP}$ may be one of the key events in transition from reversible to irreversible reperfusion injury, or cell death, during the first minutes of 
reperfusion. In fact, the opening of these channels results in a further production of ROS and mitochondrial uncoupling of oxidative phosphorylation and mitochondrial swelling, resulting in cellular apoptosis and necrosis of the myocardium. Despite a modest increase in the levels of $\mathrm{Pi}$ and mitochondrial deenergization, $\mathrm{Ca}^{2+}$ overload, and the formation of ROS during ischemia, the long-term opening of $\mathrm{mPTP}$ is antagonized by acidosis. On the contrary, and despite an initial recovery of $\Delta \psi \mathrm{m}$, on reperfusion, $\mathrm{mPTP}$ opening is strongly facilitated by $\mathrm{pH}$ recovery, increased matrix $\mathrm{Ca}^{2+}$ concentration, and accumulation of ROS (Figs. 1 and 8). This underlines the importance of the MPTP inhibition by acidosis in cardioprotection.

Actually, cardioprotective interventions induce several cytosolic signaling events resulting in suppression of the mPTP opening, but it is still uncertain how these events are conveyed to mitochondria. It has been proposed that kinases (e.g., PKC, PKG, and GSK-3 $\beta$ ) may play a direct pivotal role in the interaction with the $\mathrm{mPTP}$ after that these kinases are phosphorylated by the upstream protective signals, such as membrane receptor activation and Akt phosphorylation in the RISK pathway (e.g., 117, 137, 141, and 185) (see section IV).

\section{Transient opening of MPTP can be protective}

As mentioned above, transient opening of mPTP occurs in physiological conditions, and it can be protective $(135,308)$. Transient mPTP opening has been proposed to serve as a $\mathrm{Ca}^{2+}$-release mechanism by which mitochondria avoid matrix $\mathrm{Ca}^{2+}$ overload $(170,171,180)$. It has been also proposed that transient MPTP openings occur asynchronously in the mitochondrial population in a stochastic fashion (198). This may allow this population of asynchronously cycling mitochondria to tolerate markedly extramitochondrial $\mathrm{Ca}^{2+}$ overload without collectively depolarizing, whereas transient increased ROS production due to transient $\mathrm{MPTP}$ opening may engage cardioprotective signaling.

A recent in vitro study (169) suggested that both transient formation and inhibition of the MPTP can be considered for therapeutic purposes, and that there is a defined therapeutic window, with the first few minutes of reoxygenation being a crucial period to achieve protection. This is the first study describing direct transient opening of the pore as a possible clinical target for cardioprotection in a postischemic phase. It is necessary to confirm in future work the observations reported in this study in in vivo animal models and in humans.

Thus, the emerging picture is that similar to ROS, (i) $\mathrm{mPTP}$ opening is a two-edged sword, with both protective (transient opening) and deleterious (prolonged opening) actions in both the pre- and postischemic phases. However, this picture needs to be supported by future in vivo studies; (ii) a more substantial picture is the one that sees the transient opening of $\mathrm{mPTP}$ to be protective in the preischemic and the prolonged opening to be deleterious in the postischemic phase. Therefore, the pharmacological closure of the pores at this latter stage is considered to be highly protective. These two scenarios are not mutually exclusive.

\section{Chronic ischemia and mitochondria}

Although the review focuses on acute I/R and protection, here we will briefly consider the effects on the mitochondrial structure and function and the possible therapeutic target in chronic ischemia. For a more detailed insight into character- istics of mitochondria in chronic ischemia, we kindly refer the reader to related reviews elaborating on this point (e.g., 312).

Myocardium can respond to a prolonged decrease in perfusion by achieving a balanced state of downregulation of metabolic demand, termed "hibernating" myocardium, which is characterized by several alterations of the mitochondrial structure and function. A number of small mitochondria within the area of reversible lesions have been described in the myocardium chronically underperfused (209). Two key events have been observed after acute ischemia reperfusion and chronic ischemia. These events are the decrease of the stimulatory effect of creatine on metabolic activity and the increase of OMM permeability to Cyt $\mathrm{c}$ and ADP. These effects indicate the alteration of the intermembrane space architecture, leading to the loss of intracellular adenine nucleotide compartmentation and possibly of functional coupling of mitochondrial creatine kinase and ANT. These alterations result in the impairment of intracellular energy transfer from mitochondria to ATP-utilizing sites located in the cytosol. This may play a significant role in ischemic injury and alterations in the heart function (87). Preservation of high-energy phosphate levels and reduction in MVO2 have been observed after ischemia in chronic hibernating myocardium (160). These functional alterations are usually supported by structural alterations characterized by mitochondrial clustering and swelling associated with membrane rupture. Yet, favorable mitochondrial adaptations in a swine model of chronic myocardial ischemia have been observed. In this model, isolated mitochondria from the ischemic tissue demonstrate preserved state-3 respiration after anoxia/reoxygenation (228). This is consistent with a stress-resistant state, which is characterized by a mild degree of uncoupling under basal conditions and decreased $\mathrm{O}_{2}{ }^{-\bullet}$ generation. In fact, UCP 2 expression is enhanced in these mitochondria, providing a potential mechanism for the favorable mitochondrial adaptations (228). It may be argued that highly localized, specific mitochondrial enzyme changes (beneficial/detrimental) may result from chronic myocardial ischemia, which may make the differences between surviving/hibernating or dying tissue.

Protective strategy: the transcriptional coactivator PGC- $1 \alpha$ coactivates downstream transcription factors such as estrogenrelated receptors, nuclear respiratory factors, and peroxisome proliferator-activated receptors (PPARs). These factors are known to regulate many aspects of energy metabolism, including mitochondrial biogenesis, fatty acid oxidation, and antioxidant detoxification $(218,312)$. Since PGC- $1 \alpha$ is upstream of the regulation of energy metabolism, targeting it may represent an approach allowing an improvement of energy metabolism in multiple sites (233). Improving mitochondrial biogenesis by upregulating PGC- $1 \alpha$ is a strategy largely at work in metabolic diseases, and it has been proposed for chronic ischemic disease. PGC- $1 \alpha$ is regulated in a cardiac-specific manner (233), and more work is needed to understand its role and regulation in the normal and failing heart. Because ischemic disease is also a metabolic disease, such a strategy could be beneficial in this pathology.

\section{Cardioprotective Strategies Targeting Mitochondria in Acute I/R Injury}

It is likely that the prolonged mPTP opening is the triggering event in reperfusion injury. This hypothesis seems 
correct, because the observations with cardioprotective strategies suggest that a large percentage of cell death after I/ $\mathrm{R}$ injury results from $\mathrm{mPTP}$ formation. In fact, the current body of evidence indicates that inhibition of MPTP formation is one of the end effectors of cardioprotective strategies (135, $137,139,284)$. The importance of mPTP closure as a target for myocardial protection has been described in numerous investigations (e.g., 122, 137, and 184). However, as noted above, $\mathrm{mPTP}$ opening and closing all the time and a transient increase in opening probability of these pores may be involved in ROS-dependent cardioprotection by PreC (138, 139). Furthermore, at least two mechanisms that are not mutually exclusive have been proposed to explain the mitochondrial membrane permeabilization and apoptosis. Besides mPTP, which includes the participation of both IMM and OMM, a mechanism involving only OMM and the formation of MAC have been described [for review see $(119,194)]$. Although there is a controversy about the structure, regulation, and the precise role of these two channels, strong evidence indicates that the Bcl-2 family proteins contribute to mechanisms of both MPTP and MAC formation $(119,194)$. In fact, the Bcl-2 family regulates the intrinsic pathway of apoptosis: Bax-mediated apoptosis is regulated via constant cycling between the mitochondria and the cytosol, and retrotranslocation of Bax to the cytosol depends on interaction with Bclxl.

Mitochondria supply energy in the form of ATP. However, mitochondria can also play a destructive role and initiate cell death pathways. They can be the triggers and/or the final effectors of all forms of cell death. If mitochondria play an important role in cell death, then it is not surprising that cardioprotective mechanisms may act, at least in part, by opposing mitochondrial cell death pathways. Mitochondria are both a major site for determining the loss of cell viability and a pivotal target of processes triggered by $I / R$, such as massive elevation in $\left[\mathrm{Ca}^{2+}\right]$ and ROS events which, as said, occur mainly at the beginning of reperfusion. In fact, lethal reperfusion injury appears to represent from $20 \%$ to $70 \%$ of the total amount of the irreversible myocardial damage in experimental animals, according to the studied model and species $(175,193,329,393)$. Also in humans, part of the cardiomyocytes constituting the final infarct size dies after the onset of reperfusion $(223,332,333,353,384)$. Therefore, reperfusion injury limitation remains a major therapeutic target $(174,179,269,368,392)$. Nevertheless, it must be borne in mind that attenuation of mitochondrial redox potential, ROS production, and mitochondrial $\mathrm{Ca}^{2+}$ overload are also observed in ischemia/hypoxia $(17,49,191,363-365)$, as we will discuss in the next section.

\section{A. PreC and PostC}

As stated in the introduction, in 1986, Murry et al. (239) reported that four 5-min circumflex occlusions, each separated by $5 \mathrm{~min}$ of reperfusion, followed by a sustained $40-\mathrm{min}$ occlusion (index ischemia=infarcting ischemia) in the dog heart dramatically attenuated I/R injury. This phenomenon was named PreC. In 2003, Zhao et al. (393) reported that three episodes of $30 \mathrm{~s}$ of reperfusion/30s of ischemia performed immediately after index ischemia (60-min coronary occlusion) in the dog heart drastically attenuated reperfusion injury. This phenomenon was named PostC. It was soon clear that the later the application of the first PostC ischemia, the lower the protection (Fig. 2). The discovery of ischemic PostC provided strong evidence supporting the existence of reperfusion injury (154). It is commonly believed that cardioprotective signals mediate protection by acting on the mitochondria to inhibit mitochondrial-mediated cell death. Therefore, an understanding of the mechanisms of cardioprotection is strictly linked to an understanding of the mechanisms by which mitochondria regulate cell death.

The protective effects observed with PostC are comparable to those observed with the powerful PreC $(269,393)$. PostC has been shown to have salubrious effects on different tissue types within the heart (cardiomyocytes and endothelium) and protect against various pathological processes, including necrosis, apoptosis, contractile dysfunction, arrhythmias, and microvascular injury or no reflow. The mechanisms by which PostC alters the pathophysiology of reperfusion injury is exceedingly complex, and involves physiological mechanisms (e.g., delaying realkalinization of tissue $\mathrm{pH}$, triggering release of autacoids, redox signaling, and opening and closing of various channels) and molecular mechanisms (especially activation of kinases of the RISK and SAFE pathways; see also below) that impact on cellular and subcellular targets or effectors $(260,367,393)$.

The term "PostC" has highlighted the importance of intervening at the beginning of myocardial reperfusion to protect the postischemic heart. This is a clinically relevant timepoint for intervention in patients presented with AMI. As such, its clinical application has been rapid for both STEMI patients undergoing primary PCI (e.g., 333 and 356) and for patients undergoing on-pump cardiac surgery (223) (see also section XI).

Intensive investigation of the signaling pathways underlying PreC and PostC has identified a number of signal transduction pathways conveying the cardioprotective signal from the sarcolemma to the mitochondria, some of which are common in PreC and PostC. In fact, both PreC and PostC induce activation of signaling elements during the early reperfusion after the index ischemia (90) (Figs. 3 and 11). In this phase, a great attention has been focused on the cGMP/PKG pathway (e.g., 153, 268, and 271), on the RISK pathway (e.g., 132,139 and 326), which involves the kinases Akt and ERK1/ 2 , and more recently, on the SAFE pathway that contributes to PostC protection through the activation of TNF- $\alpha$, its receptor type-2, JAK, and STAT-3 (e.g., 137 and 212). All these pathways in PreC and PostC converge on mitochondria via the modulation of several kinases, including GSK-3 $\beta$, Bcl-2/Bax/ $\mathrm{Bad}$, and $\mathrm{PKC} \varepsilon$ (e.g., 137 and 236). In Figure 9 are reported only the principal modalities of mitochondrial control by cytosolic kinases. Nevertheless, these modalities are still controversial $(66,67,113,296)$.

As said, MPTP has been shown to open at the start of reperfusion, and its opening is generally attributed to an overload of matrix $\mathrm{Ca}^{2+}$ and/or ROS increase. Therefore, it is likely that cardioprotective signaling reduces matrix $\mathrm{Ca}^{2+}$ and/or ROS, rather than by direct modulation of mPTP components. However, redox signaling and acidosis in early reperfusion are two cardioprotective processes operating in the very early reperfusion in both PreC and PostC (Figs. 3 and 11). Therefore, these two processes may act, first, directly on mPTP components, limiting their opening and then may activate signaling pathways that have been suggested to 


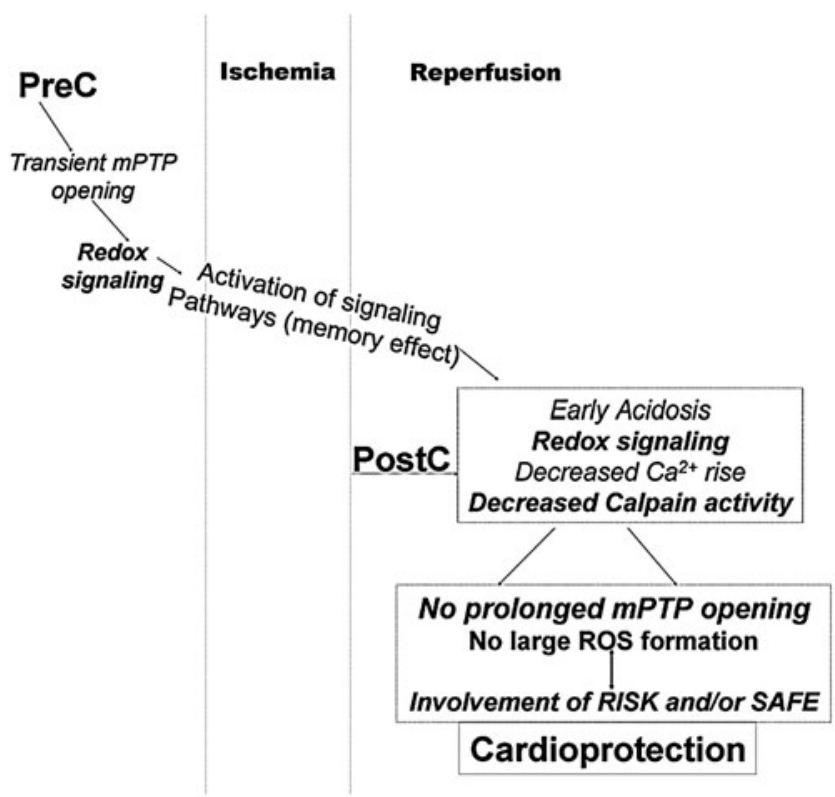

FIG. 11. Key mechanisms proposed for PreC- and PostCinduced decrease in susceptibility to MPTP opening during reperfusion. Key events are transient physiological mPTP opening in the preischemic phase and acidosis plus redox signaling in reperfusion. These events avoid prolonged mPTP opening, allow the activation of cardioprotection pathways, and lead together to MPTP inhibition to the prevention of cell death and organ protection. For other acronyms, see the list of Abbreviations Used.

converge again on mitochondria, definitely decreasing susceptibility to $\mathrm{mPTP}$ opening and mediating protection. More details on these two processes are given in the next sections.

\section{B. Redox signaling and acidosis in early reperfusion}

We must consider the detrimental effects of ROS within the heart, before discussing the beneficial role of redox signaling.

1. Detrimental effects of excessive ROS. Various detrimental processes can be the result of an imbalance between the formation of ROS/RNS and limited antioxidant defenses (referred to as oxidative stress). For instance, excessive reactive species indiscriminately react with DNA, lipids and proteins. These are complex processes, in brief: a radical reaction with DNA may oxidize DNA bases such as 8hydroxydeoxyguanosine leading to mutation and DNA strand breaking; reaction with lipids gives rise to peroxyl and alkoxyl radicals, leading to lipid peroxidation; reaction with amino acid residue side chains of proteins form protein carbonyls; and reaction with methionine forms methionine sulfide $(18,362,391,397,399)$. The lack of protection of mitochondrial DNA by histones, the limited capacity of repair mechanisms, and the proximity of mitochondrial DNA to the production site of ROS by RIRR render the mitochondrial DNA highly susceptible to increased oxidative stress (120, 316). Excessive oxidative stress, besides contributing to irreversible myocardial injury, inducing prolonged mPTP opening, and leading to cellular dysfunction and cell death, may also induce reversible injury during ischemia and during reperfusion $(17,206,300,363)$. As said above, oxidative stress occurring during ischemia may be also important in reperfusion injury $(20,49,364,365)$. Moreover, the reversible contractile dysfunction after myocardial I/R (stunning) is clearly a manifestation of oxidative stress (125). Whether stunning is due to RIRR has not been investigated yet.

ROS scavenging by administration of MPG and phenanthroline at reperfusion reduces cell death of embryonic chick cardiomyocytes, confirming that the burst of ROS at the onset of reperfusion may be causal for cell death (367). In contrast, ROS scavenging by a combination of catalase and SOD during reperfusion did not reduce the infarct size in dog hearts in vivo; however, microvascular injury and the low-reflow phenomenon were attenuated (289). On the other hand, reperfusion plus SOD limited the myocardial infarct size in a closed-chest pig model (244). All these contrasting results reveal the complexity of the redox system. Moreover, low levels of reactive species may act as secondary messengers, modulating cardioprotective signaling pathways by a covalent modification of target molecules, referred to as redox signaling.

Therefore, the key message here is that ROS/RNS effects may range from beneficial to reversible and even irreversible damages mainly depending on reactive species amount, timing, and compartmentalization.

2. Beneficial effects of redox signaling and acidosis. It has been proposed that reintroduction of oxygen after ischemia induces ROS production, but it does not protect against reperfusion injury, because $\mathrm{mPTP}$ opens triggering RIRR before the activation of endogenous survival pathways.

It has also been proposed that both ischemic PreC and PostC protect, because they maintain acidosis during early reoxygenation at the beginning of reperfusion, which inhibits mPTP formation and allows ROS signaling, so that during early reperfusion, acidosis and ROS signaling avoid cell death and give the heart enough time to activate the protective signaling pathways (e.g., RISK); thus, we can consider that the heart preconditions itself against reperfusion injury $(61,90$, $133,135,137,140$ ) (see also below). Therefore, a key event for cardioprotection may be prolongation of cellular acidosis by cardioprotective phenomena during early reperfusion (Figs. 3 and 11). In fact, both acidic infusion in early reperfusion or PostC and delaying $\mathrm{pH}$ normalization could inhibit mPTP during the first minutes of reflow and allow for endogenous protective signaling pathways to be activated by a small quantity of ROS (redox signaling). The delivery of oxygen during acidic perfusion or the brief intermittent reperfusions of PostC would promote mitochondrial ROS formation in a small amount, which has been proposed to activate isoforms of PKC (62). PKC appears as a critical kinase in the signaling cascade, leading to a reduced probability of $\mathrm{mPTP}$ opening (see below) after $\mathrm{pH}$ normalization $(276,388)$.

Hence, not only mPTP may be inhibited by redox signaling and acidosis but also a transient opening of the MPTP has been suggested to induce a slight, transient formation of ROS that might be relevant for cardioprotection $(21,79,139,171$, 282 , 283). Supporting this concept, pharmacological and genetic inhibition of Cyp-D was reported to abrogate both PreCinduced ROS formation and protection $(142,135,141)$.

We were among the first to demonstrate that the ROS scavengers $N$-acetylcysteine (NAC) and MPG prevent the protective effects of ischemic or pharmacological PostC (271, 
$272,361,382)$, and the same ROS scavenger has been shown to block the protection afforded by acidic reperfusion $(64,275)$. In our laboratory, isolated rat hearts were subjected to 30-min ischemia and 120-min reperfusion, and PostC at onset of reperfusion by five cycles of 10-s ischemia and reperfusion each significantly reduced the infarct size. While the protection by PostC was lost in hearts perfused with NAC for the entire 2-h reperfusion period, the infarct size was still reduced when, in postconditioned hearts, the perfusion with NAC was initiated after the first $3 \mathrm{~min}$ of reperfusion, demonstrating an essential role of ROS formation during early reperfusion in PostC protection (276). Reperfusion of isolated rabbit hearts with an acidic buffer for the first 2 min of reperfusion significantly reduces the infarct size. This cardioprotection is blocked by co-infusion with MPG (64), suggesting the involvement of ROS signaling in acidosis-induced protection. Infarct size reduction by PostC in mouse hearts in vivo was attenuated by the ROS scavenger MPG when administered few minutes before, but not $10 \mathrm{~min}$ after the PostC stimulus (361). In particular, in mice, pharmacological PostC by either $1.4 \%$ isoflurane or $10 \mathrm{mg} / \mathrm{kg}$ of the delta-opioid receptor agonist SNC121 was also abolished by MPG when applied immediately before, but not $10 \mathrm{~min}$ after reperfusion (361). NAC for the first minutes of reperfusion also abolished PostC by bradykinin or sevoflurane in isolated rat hearts (271). Importantly, in human myocardium, desflurane-induced PostC was mediated by adenosine and bradykinin receptors via a ROS signaling (214). These data support a central role for ROS signaling during early reperfusion in the protection by ischemic PostC and in the protection by acidosis in early reperfusion. It is generally thought that ROS generation at the beginning of reperfusion is mainly mediated by an enhanced electron leak in the ETC due to ischemic oxidative damage with an important role for the opening of $\mathrm{mK}_{\mathrm{ATP}}$ channels. It has also been suggested, and we have obtained some data to support the concept, that PostC might lead to increased SNO of proteins that might protect them from oxidation (275). Indeed, acidosis may favor $\mathrm{NO}^{\bullet}$ production via breakdown of nitrite (401) and via kininogenase activation and bradykinin formation, which may activate NOS (360). Acidosis may also downregulate SOD activity, whose optimal $\mathrm{pH}$ is between 7.4 and 7.8 (264). Since SOD is a denitrosylating agent (252), acidosis may contribute to elevation of nitrosylated proteins (275), including proteins involved in regulation of mitochondrial energetics and calcium transport $(275,340,341$; and unpublished observations of the authors).

In summary, in protected reperfusion, low levels of ROS/ RNS might act directly on mPTP components or activate signaling pathways that have been suggested to act on mitochondria, decreasing their susceptibility to prolonged $\mathrm{mPTP}$ opening. Therefore, redox signaling by transient/reduced formation of ROS/RNS is also included among the triggers of PostC $(274,275)$.

\section{Timing and Targets of ROS Signaling in Cardioprotection}

It is now clear that inhibition of mPTP opening by cardioprotection is central for the reduction of oxidative stress. This may be achieved by mitochondrial protein phosphorylation as well as by the action of redox signaling. In fact, in the context of cardioprotection, ROS/RNS with a signaling role may play a pivotal role. ROS/RNS generated during ischemia and/or reperfusion, in quantities below those that damage the heart, may activate mediators that trigger cardioprotection. Therefore, protective ROS/RNS are suggested to be formed during three time points: (i) during PreC-ischemia and/or (ii) during reperfusion that follows the brief PreCischemia, and (iii) in protected hearts, in the initial part of reperfusion that follows the index ischemia: both (iiia) in PostC and (iiib) in PreC context (Fig. 3).

It may be worthwhile to recall that without appropriate PreC and PostC interventions, ROS/RNS generated at the beginning of reperfusion are usually lethal, and not the ROS/ RNS that would trigger protection (points iiia and iiib, respectively). Moreover, while ROS scavenging attenuates the infarct size reduction by ischemic PostC or diazoxide, increasing exogenous ROS formation at the onset of reperfusion does not confer protection (271). Since pharmacological generation of endogenous ROS/RNS or administration of exogenous ROS/RNS (61-63, 191, 251, 262, 382, 387) before index ischemia can trigger PreC, it is likely that the type, the concentration, and/or the compartmentalization of endogenous ROS/RNS generation may play a pivotal role in triggering protection at the reperfusion time (after index ischemia). In fact, it must be borne in mind that both PreC and PostC may involve different subcellular compartments that may interact, such as the sarcoplasmic reticulum, mitochondria, and the nucleus $(135,259,390)$. Actually, the activation of Akt may recover the impaired cardiac contractile function, induced by the endoplasmic reticulum stress via GSK-3 $\beta$-mediated suppression of mPTP opening, showing a cross-talk among these organelles (390). Moreover, while the cytoplasm is made acidotic by PreC and PostC, $\mathrm{mK}_{\mathrm{ATP}}$ channel opening induces mitochondrial matrix alkalinization $(66,67,113,296)$. Finally, while $\mathrm{O}_{2}^{-}$and $\mathrm{NO}^{\bullet}$ lead to $\mathrm{ONOO}^{-}$increase, $\mathrm{ONOO}^{-}$may decrease by secondary reaction between $\mathrm{NO}^{\bullet}$ and $\mathrm{ONOO}^{-}$ itself, leading to $\mathrm{N}_{2} \mathrm{O}_{3}$; the latter condition may occur in the protected heart when $\mathrm{NO}^{\bullet}$ formation is facilitated $(275,354)$.

(i) A wide body of evidence exists demonstrating that an appreciable formation of ROS occurs during ischemia $(17,191,300,334,364,365)$. In fact, mitochondrial ROS formation is favored by a decrease in the electron flow resulting from ETC inhibition and is counteracted by uncoupling that is generally produced by an increased IMM permeability to protons. Therefore, the inhibition of ETC caused by the insufficient oxygenation facilitates the escape of electrons that can react directly with the scarce available oxygen, resulting in ROS formation. A significant increase in ROS production in isolated hearts during brief PreC ischemia and during the index ischemia has been reported (191). These findings are supported by observations in isolated cardiomyocytes and other studies in the isolated perfused heart model $(17,50,334,364)$. Therefore, it has been suggested that ROS released during the brief PreC ischemia, and not on reperfusion, lead to activation of cardioprotective pathways (191).

(ii) Small amounts of ROS/RNS may be formed during reperfusion after a short period of PreC ischemia (63, 89, 140). MPG is a cell-permeant ROS scavenger that removes $\mathrm{OH}^{\bullet}$ and $\mathrm{ONOO}^{-}$very effectively and blocks the protective effects of PreC (e.g., 67). Based on these 
considerations, it was tested whether the ROS/RNS that triggers protection are produced during the ischemic or the reperfusion phases of the PreC maneuvers (89). It was concluded that protective redox signaling occurs when molecular $\mathrm{O}_{2}$ is reintroduced after the brief PreC ischemia, with the contribution of transient mPTP opening $(89,140)$.

Nevertheless, it is not easy to solve whether are more important ROS produced during brief PreC ischemia or during the following reperfusion to trigger protection. It has been postulated that ROS produced during ischemia play a role in in vitro models (236). However, it has also been suggested that $\mathrm{O}_{2}{ }^{-\bullet}$, produced during ischemia, may be converted to downstream products such as $\mathrm{H}_{2} \mathrm{O}_{2}$ and $\mathrm{OH}^{\bullet}$ on reperfusion, and that these species can trigger PreC (191).

(iiia) The above observations were done in the PreC phase, that is, before the index ischemia and extended to PostC itself $(142,141)$. In fact, as reported above, the PostC protective effect was abrogated by infusing during early reperfusion a broad spectrum of ROS scavengers, making the oxygenated perfusate alkaline during the early reperfusion phases or making the early reperfusion buffer hypoxic $(62,64$, 111, 175, 271, 276).

(iiib) Clearly, both PreC and PostC induce activation of signaling elements (RISK and SAFE) during the early reperfusion after the prolonged index ischemia (90, 134, 137) (Fig. 11). It is now thought that after a triggering phase in a preischemic period, the actual protection by PreC occurs in the reperfusion rather than the ischemic phase, with the repopulation of sensitized G-protein-coupled receptor at the beginning of myocardial reperfusion after the index ischemia (61). Hence, reintroduction of $\mathrm{O}_{2}$ at the beginning of reperfusion permits generation of signaling ROS, and this will activate the PKC-dependent signaling cascade. As it has been observed for the PostC protective effect, also PreC protection was abrogated by infusing large-spectrum ROS scavengers, making the oxygenated perfusate alkaline or making the buffer hypoxic during early reperfusion $(61,134,139)$.

\section{A. ROS/RNS signaling may be modulated by antioxidants}

Reactive species function as trigger molecules of protection by activating protein kinases such as MAPK and AMPK, as well as PKC, within and outside the mitochondria, including PKC $\varepsilon(68,72,199,260,262,270,288,341)$ and the MAPK p38 and/or JAK/STAT (387). As said, several mitochondrial components are targeted by ROS/RNS via oxidative/nitrosative processes. Accordingly, many large-spectrum scavengers of ROS/RNS, such as ascorbic acid, MPG, or NAC, attenuate infarct size reduction by ischemic or pharmacological PreC or PostC, in several animal species (e.g., 109, 253, 260, $262,269,270$, and 276). Since a target of ROS/RNS in redox signaling is the PKC $(199,260,288,339,341)$, the hearts can be preconditioned by simply infusing free-radical generators into the coronary arteries, and the resulting protection can be blocked by either a PKC antagonist or a ROS scavenger (61,
358, 382). Evidence exists that ROS-activated PKC will also protect the reperfused heart $(262,276)$. This sequence would explain the observation that the PKC activator could rescue hearts experiencing acidic and hypoxic reperfusion (62). Moreover, chelerythrine, a wide-spectrum PKC antagonist, blocks PostC protection $(276,388)$.

Indeed, though it has been reported that ROS/RNS can activate kinases, and ROS/RNS scavenger may attenuate cardioprotective pathways, recently, we have observed in an ex vivo study that PostC and acidosis induce downregulation of SOD, whereas catalase activity does not change in the early reperfusion phase (275). Moreover, PostC reduces 3nitrotyrosine (3-NT) and increases S-nitrosylated protein levels, thus contributing to cardioprotection triggering (275). Indeed, in addition to activating cGMP/PKG-dependent signaling pathways, $\mathrm{NO}^{\bullet} / \mathrm{RNS}$ can modify sulfhydryl residues of proteins through their $\mathrm{SNO}$, which has emerged as an important post-translational protein modification. Thus, SNO of proteins is the result of a transnitrosylation reaction, or alternatively, donors of $\mathrm{NO}^{+}\left(e . g ., \mathrm{N}_{2} \mathrm{O}_{3}\right)$ can nitrosylate a cysteine moiety. SNO of mitochondrial proteins has attracted the attention of researchers in cardioprotection $(62,64,112$, $175,252,274,275)$. Therefore, SNO has been proposed to be very important in both ischemic PreC and PostC cardioprotection, limiting oxidative stress. As such, SNO of proteins can be seen as a result of redox signaling, leading to a potentiation of the antioxidant system.

\section{B. Role of SNO in regulating mitochondrial function in $I / R$ and cardioprotection}

Within the context of redox signaling, the increase in $\mathrm{NO}^{\bullet}$ occurring during PreC $(18,401)$ or PostC $(173,205,262,274-$ 276) can lead to an increase in SNO of several mitochondrial proteins $(47,340)$. These are a subproteome of SNO proteins (see below), which include complex I (47) and other proteins involved in mitochondrial energetics, calcium transport, and metabolism (340). Intriguingly, induction of the mitochondrial permeability transition by N-ethylmaleimide has been seen to depend on secondary oxidation of critical thiol groups. In fact, it has been reported that $\mathrm{mPTP}$ putative components (see above) may have several important cysteines residues, and that mPTP opening is blocked by low levels of N-ethylmaleimide $(21,79)$. Depending on the $\mathrm{NO}^{\bullet}$ level, these cysteines may be nitrosylated and the activity of $\mathrm{mPTP}$ regulated. Furthermore, SNO of proteins is rapidly reversible so that changes would be rapidly reversed on reperfusion. It has been reported that cardioprotection by PreC or by treatment with the naturally occurring transnitrosilating agent Snitrosoglutathione (GSNO) resulted in an increase in SNO of several mitochondrial proteins involved in regulation of mitochondrial energetic and calcium transport, including acylCoA dehydrogenase, alpha-ketoglutarate dehydrogenase, complex $\mathrm{I}$, creatine kinase, $\mathrm{F}_{0} \mathrm{~F}_{1}$-ATPase $\alpha 1$ subunit, and malate dehydrogenase (340). It has been also shown that PreC results in SNO of complex I, which may result in less ROS generation in the setting of ischemia and reperfusion $(46,47)$. We have shown that also PostC results in SNO of mitochondrial proteins (275). For further discussion on the importance of SNO in cardioprotection, we kindly refer the reader to related articles elaborating on this point (e.g., 260, 274, 340, and 341). 


\section{Preservation of Functional and Morphological Integrity of Mitochondria by PostC}

$\mathrm{I} / \mathrm{R}$ and cardioprotective interventions impact on the mitochondrial morphology and the intermitochondrial network, for example, fusion and fission (254). Mitochondrial fusion proteins include the OMM proteins mitofusin (Mfn) 1 and 2 and the IMM protein optic atrophy protein 1 (Opa1). Mitochondrial fission proteins are the dynamin-related protein 1 (Drp1), which are recruited from the cytosol to the mitochondria, and the OMM protein human mitochondrial fission protein 1 (hFis1) (84). Since a Drp1 inhibitor reduces cardiomyocyte death after $I / R$, decreasing mitochondrial fission is considered cardioprotective (255). Mfn2 is localized at the contact sites between the mitochondria and the endoplasmic reticulum, and the deficiency in Mfn2 reduces mitochondrial $\mathrm{Ca}^{2+}$ uptake by increasing the distance between the endoplasmic reticulum and the mitochondria (76). It is known that Mfn1 and Mfn2 can each compensate to a certain degree for the loss of the other. This may explain the absence of severe mitochondrial dysfunctions with a loss of Mfn2; however, it does not explain the improved recovery from ischemia and the observed reduction in ROS. Nevertheless, the inhibition of either mitochondrial fission or fusion limits the release of proapoptotic factors and excessive ROS formation from damaged mitochondria, which otherwise, may enhance postischemic cardiomyocyte injury $(76,254,255)$. On the other hand, mitochondrial fission and fusion may be viewed as a mitochondrial repair mechanism. In fact, irreversibly damaged mitochondria are removed by mitophagy, and indeed, the elimination of damaged mitochondria by mitophagy may be cardioprotective (see section IX).

PreC and PostC activate cardioprotective pathways that are protective against reperfusion injury via preservation of functional and morphological integrity of mitochondria, even in the postischemic phase. With regard to the PostC effect against apoptosis, it has been suggested that it is mediated by reduced generation of $\mathrm{O}_{2}^{-}$, lowered activity of JNKs/p38, lowered levels of caspases 3 and 8, reduced release of TNF- $\alpha$, and by the modulation of the $\mathrm{Bax} / \mathrm{Bcl}-2$ ratio (338). PostC increases the levels of antiapoptotic markers, including the cardioprotective kinase Pim-1, decreases the proapoptotic markers (e.g., Cyt c), and preserves the mitochondrial structure. In fact, at the onset of abrupt reperfusion, mitochondria undergo profound structural alterations. In particular, postischemic mitochondria are characterized by disruption of membranes, broken cristae, and the appearance of dense granules within the mitochondrial matrix, which are caused by massive accumulation of a $\mathrm{Ca}^{2+}$-generating insoluble calcium phosphate precipitate (321). These mitochondrial damages are reduced by PostC (274). Carbonylation of mitochondrial proteins was prevented, and aconitase activity was preserved in the PostC hearts, suggesting that mitochondrial integrity was associated with a diminution in oxidative stress (65).

Although PostC affects structural features of mitochondria, it does not influence mitochondrial respiration (261). In particular, PostC does not affect basal state-4 or ADP-stimulated state- 3 respirations, excluding uncoupling or inhibition of the respiratory chain as a mechanism of mPTP inhibition (5). Nevertheless, while basal respiration was not affected, ADPstimulated respiration was increased after pharmacological
PostC with morphine (249). This last observation is in line with many reports showing that a mild degree of mitochondrial dysfunction confers protection against I/R injury (e.g., 288). It may seem paradoxical that postischemic mitochondrial dysfunction does not add injury, but confers protection. In our opinion, this is the basis of the concept that cardioprotection is afforded by a partial degree of mitochondrial dysfunction leading to ROS signaling. In fact, when oxygen supply is re-established after a prolonged ischemia, if something (e.g., acidosis and CsA) prevents prolonged mPTP opening and RIRR, the partial mitochondrial dysfunction may allow ROS signaling.

Taken as a whole, ischemic and pharmacological cardioprotective interventions inhibit $\mathrm{MPTP}$ opening, prevent the loss of mitochondrial membrane potential, allow ROS signaling, preserve mitochondrial morphology, and affect fission and fusion; it is less clear whether or not PostC results in alterations of mitochondrial respiration.

\section{Summary of PreC and PostC Pathways United at Reperfusion}

While excessive ROS/RNS formation during reperfusion, which follows infarcting ischemia, enhances cell death, ROS/ RNS signaling during early reperfusion is essential for the protection by ischemic and some pharmacological PreC and PostC against reperfusion injury. As shown in Figure 3, ROS / RNS signaling before index ischemia, that is, during brief PreC ischemia and/or during the following reperfusion, is clearly involved in the triggering of PreC protection. ROS/ RNS signaling is also involved in the mediation phase of PreC and in the triggering of PostC (points iiia and iiib discussed in the section V). Opening of $\mathrm{mK}_{\mathrm{ATP}}$ channels may involve $\mathrm{Cx} 43$ and may be upstream of ROS/RNS signaling. Cardioprotective procedures delay the postischemic recovery of intracellular $\mathrm{pH}$ that might prevent $\mathrm{mPTP}$ opening directly and indirectly (i.e., by inhibiting calpain activation). In addition, mPTP opening might be further prevented by a ROS/RNS signaling that appears to depend on acidosis, which may favor $\mathrm{NO}^{\bullet}$ production and protein $\mathrm{SNO}$. Redox signaling triggers a protective kinase cascade, including $\mathrm{PKC}$, and converging on $\mathrm{mPTP}$, so that $\mathrm{MPTP}$ closure may be dependent on ROS/RNS-signaling effects, both upstream, together acidotic effect, and downstream, depending on kinase effects.

Therefore, mitochondria are involved at least in four different steps to limit reperfusion injury

(i) as targets of acidosis (in terms of prevention of mPTP opening);

(ii) as triggers or signal amplifiers (in terms of activation of $\mathrm{mK}_{\mathrm{ATP}}$ channels and the resulting formation of small amounts of ROS);

(iii) as targets of signaling pathways and end effectors (in terms of inhibition of mPTP opening and of release of proapoptotic factors into the cytosol); and

(iv) as targets of damage and protection from it (in terms of their functional and morphological integrity).

Overall, while ischemia and reperfusion damage mitochondria (e.g., limited oxidative phosphorylation), mitochondria themselves may contribute to myocardial injury (e.g., mPTP opening-induced cell death) and protection (e.g., limiting $\mathrm{MPTP}$ opening via redox signaling). 


\section{The Second Window of Protection}

The present review mainly focuses on PostC described for the first time by the Vinten-Johansen group (393) and on the so-called early PreC discovered by Murry et al. (239). Early PreC lasts $2-3 \mathrm{~h}$ and protects against infarction and arrhythmias, but little or nothing against myocardial stunning. However, as said in the introduction, the PreC maneuvers also trigger a late phase of PreC 12-24h later (Fig. 2). This late phase is also called SWOP and lasts 3 to 4 days and protects mainly against arrhythmias and stunning, but less against infarction $(266,342,348,366)$. SWOP is a polygenic phenomenon that requires the activation of multiple stressresponsive genes $(35,37)$. Therefore, while the early PreC is caused mainly by rapid post-translational modifications of pre-existing proteins, the late PreC requires the synthesis of new cardioprotective proteins, which explains the time course of this late phenomenon, with optimal effects, 24 to $48 \mathrm{~h}$ after PreC treatments (345). Actually, SWOP may be induced by various pharmacological agents (e.g., adenosine and opioid receptor agonists, NO donors, cytokines, and prostacyclin derivatives) given 24 to $72 \mathrm{~h}$ before an infarcting I/R protocol $(37,151)$. Yet, SWOP appears to be a universal response of the heart to stress in general. Besides cytokines, SWOP can be triggered by heat, stress, and exercise $(35,266,323,342,346)$. For instance, we reported that exercise-induced myocardial ischemia may trigger an adaptive response that improves the cardiac performance in a subsequent episode of exercise in patients with stable angina (69).

To explain the SWOP, it has been suggested that a sublethal ischemic stress may induce the release of chemical signals (such as $\mathrm{NO}^{\bullet}, \mathrm{ROS} / \mathrm{RNS}$, and adenosine) and may trigger a complex signaling pathway that includes the activation of PKC, Src protein tyrosine kinases, and nuclear factor $\kappa \mathrm{B}$ $(\mathrm{NF} \kappa \mathrm{B})$. PreC ischemias also activate JAK1/2, followed by recruitment of STAT-1 and STAT-3. These pathways culminate in increased synthesis of aldose reductase, cyclooxygenase-2 (COX-2), inducible NOS (iNOS), MnSOD, and possibly other cardioprotective proteins, which then mediate the protective effects of SWOP $(33,35-37,346)$.

The obligatory role of STAT-3 has been recently confirmed in SWOP, using a novel inducible cardiomyocyte-restricted STAT-3-deficient mouse. This study suggested that STAT-3 activation is important in inhibiting both the receptor pathway and the mitochondrial pathway of apoptotic death (36). Therefore, the protection by both early and late PreC as well as by PostC involves an activation of STAT-3 and is dependent on STAT-3 levels. While the cardioprotective effect of SWOP is clearly mediated by an increase in transcription-mediated protein synthesis, early PreC seems independent of gene transcription, supporting a role of STAT-3 independent of transcriptional regulation (see also above, section II.A.3).

Common endpoints in the mechanisms of early and late PreC have been identified at the mitochondrial level, including the opening of $\mathrm{mK}_{\mathrm{ATP}}$ channels (349) and the protection against mPTP opening in late PreC $(297,346)$. This goes along with an increased expression of Bcl-2 in the late phase. Bax/ Bak, members of the Bcl-2 family, may interact with mPTP directly, thereby facilitating pore opening. This interaction of Bax/Bak with mitochondria can be inhibited by Bcl-2 increases in late PreC, suggesting functional involvement of Bcl-2 in depression of mPTP opening $(297,346)$. Bolli and coworkers (311) have also studied the combination of late PreC and PostC in a chronically instrumented rat model. The animals underwent ischemic PreC $24 \mathrm{~h}$ before an infarcting coronary occlusion and were or were not subjected to PostC maneuvers at the onset of reperfusion after the occlusion. Experiments were performed in the presence of the inhibition of COX-2. These authors concluded that the combination of late PreC and PostC produces additive protection; this is likely due to a PostC-induced enhancement of COX-2 activity. This is in line with our findings that protection by PostC and bradykinin requires COX activation and prostacyclin release during reperfusion (273). For further information on SWOP, the reader is kindly redirected on extensive reviews on this topic (e.g., 35, 323, 342, and 346).

\section{Autophagy and Mitophagy}

Macroautophagy (called also autophagy) is a process present in normal organs and may be involved in cardioprotection. It is a regulated intracellular catabolic process that serves as the quality-control mechanism of cells. Autophagy regulates the disposal of damaged and dysfunctional protein aggregates and organelles, including mitochondria. In fact, the selective autophagic targeting and clearance of damaged mitochondria is a phenomenon termed "mitophagy" (213). The molecular machinery of auto- and mitophagy has been described in several recent reviews (e.g., 85, 115, 116, 196, 290, and 347).

It has been suggested that autophagy is essential for ischemic PreC-induced protection (163). These authors have also reported that upregulation of autophagy is involved in the cardioprotection achieved with pharmacological PreC using different pharmacologic agents, including an adenosine $\mathrm{A}_{1}$ receptor agonist (2-chlorocyclopentyladenosine), an opener of $\mathrm{mK}_{\mathrm{ATP}}$ channels (diazoxide), a sodium channel blocker (ranolazine), a cytochrome P450 inhibitor (sulfaphenazole), and a purinergic receptor agonist (UTP) $(162,163)$. Moreover, upregulation of autophagy has been suggested to play a causal role in the infarct-sparing effect of PostC (371). Autophagy plays also a role in the cardioprotective mechanisms developed by repetitive ischemic PreC in a swine model (77). This experimental model may be more relevant to patients with chronic ischemic heart disease, who are subjected to repetitive episodes of ischemia.

Most recently, research attention has focused on mitochondrial function and mitophagy. Actually, mitophagy may be relevant to more than just PreC and PostC; it could be an important element in lifespan extension, where periodic removal of damaged mitochondria would allow their replacement with new and more efficient ones (118). Moreover, upregulation of autophagy has been associated with preservation of mitochondrial membrane potential and sarcolemmal membrane integrity and a resultant significant delay in the onset of irreversible cell injury (both apoptotic and necrotic cell death) (222). Within the context of cardioprotection, recent data have demonstrated that upregulation of mitophagy plays a pivotal role in the reduction of the infarct size achieved with PreC (161). In transgenic mice, obtained with genetic deletion of two molecular inhibitors of mitophagy, p53 and TP53-induced glycolysis and apoptosis regulator (TIGAR), it has been observed an upregulation of mitophagy. In this model, it has been obtained a reduction of the incidence 
of apoptotic cell death and has been observed a favorable effect on cardiac remodeling, after permanent coronary artery ligation in vivo (159). Moreover, treatment of $\mathrm{p} 53^{-/-}$and TIGAR $^{-/-}$mice with chloroquine (an agent that inhibits formation and/or degradation of autophagolysosomes) abrogated the cardioprotection conferred by deletion of p53 and TIGAR, an effect accompanied by reduced mitophagy and the accumulation of damaged mitochondria in the ischemic myocardium (159). These data support long-term beneficial effects for autophagy and mitophagy in the attenuation of adverse postinfarction left ventricular remodeling.

Taken together, these data suggest that upregulation of autophagy and mitophagy renders myocardium resistant to ischemic injury and may have long-term beneficial effects. However, it has been also reported that upregulation of autophagy in I/ $\mathrm{R}$ is detrimental and exacerbates, rather than limits, myocardial injury. For instance, it has been observed that classical ischemic PreC is not associated with upregulation of autophagy in the in vivo pig model (380), and that the protective effects of PostC are accompanied by a limitation in (rather than augmentation) Beclin-1 expression in an in vitro model (88). In fact, Beclin-1 may be used as tool to determine whether autophagy has been up- or downregulated by various stimuli [for review see (196)]. An interesting study is that of Ma and coworkers (224), who have shown that ALDH-2 overexpression moderates, via an AMPK mechanism, the upregulation of autophagy in the ischemic phase and limits autophagy, via an Akt mechanism, in the reperfusion phase. These authors suggested that the combination of these two actions in ischemia and reperfusion may be responsible for the cardioprotective effect of ALDH-2 against I/R injury.

The inconsistency of data on the role of autophagy may depend on multiple factors, including magnitude, timing, and involved cell type in the process. The interconnections among these multiple factors are at present incompletely understood. With no doubt, as an alternative process of cardioprotection, autophagy merits further study.

\section{Comorbidities, I/R, and Cardioprotection}

Before taking into account, the transition from research laboratory to the clinic of the concept of mitochondria as the main actors in the signals of life and death, it is worth to discuss the changes in the mitochondrial function in some comorbidities that can accompany the I/ $\mathrm{R}$ in humans. In fact, ischemic heart disease in humans is a complex disorder caused by or associated with known cardiovascular risk factors, including aging, hypertension, myocardial hypertrophy, and/or MS (i.e., obesity, hyperlipidemia and diabetes). These conditions may be confounders in the outcome of cardioprotection. Moreover, although not a comorbidity, sex may be a complicating factor: in different animal models, the magnitude of I/R injury seems reduced, but the cardioprotection of conditioning stimuli seems attenuated in adult females versus males $(104,152,235,269,277)$.

\section{A. Mitochondria and MS}

Since the MS is an archetypal condition with an altered mitochondrial function, and since it has an increasing prevalence in the patients with ischemic cardiovascular disease (97), this condition will be considered with more details. Other risk factors of heart diseases are briefly discussed; for further details, the reader may see $(104,259,368)$.

MS is now defined as a complex syndrome, which includes several interrelated disturbances of glucose and lipid homeostasis, which may lead to diabetes (108). Since the MS induces a significant variation of $\mathrm{O}_{2}$ consumption, alterations in the mitochondrial function and mitochondrial morphology play a major role in this syndrome (43). Several studies associated mitochondrial dysfunction and the MS; for example, Wisløff et al. (377) proposed an association between low expression of the protein involved in mitochondrial function, in the skeletal muscle, with the occurrence of multiple cardiovascular risk factors (insulin resistance, hyperlipidemia, and hypertension) in rats with low oxidative capacity. In humans, for instance, Peterson et al. (281) found that the increase in Body Mass Index was associated with an increase in myocardial $\mathrm{VO}_{2}$, reduced cardiac efficiency (ratio of cardiac work to $\mathrm{VO}_{2}$ ), and impaired glucose tolerance correlating with increased fatty acid utilization. A reduced cardiac phosphocreatine/ATP ratio, an impaired high-energy phosphate metabolism, and a cardiac energy deficit have been reported (314).

In diabetic and obese individuals, the increased myocardial fatty acid oxidative capacities are mediated, at least in part, by increased activity of PPARs (43), which are regulators of fatty acid oxidation in the heart. In particular, PPAR $\alpha$ increases the expression of genes involved in several steps of cardiac fatty acid utilization (11). It has been reported that either high-fat diet or streptozotocin-induced diabetes may increase UCP expression in the striated muscles $(54,187)$. This increased expression may play a pivotal role in supporting increased oxidative stress. Moreover, type- 2 diabetes patients have been shown to possess reduced ETC capacities and reduced citrate synthase activity $(189,316)$. The preferential metabolism of fatty acids, reducing glucose utilization (280), causes gradual dysfunction of pancreatic beta and other cells and upregulation of mitochondrial UCP2 and UCP3, thus resulting in an increased uncoupling of mitochondrial respiration, a reduced ETC activity, and ATP production, with subsequent skeletal muscle fatigue $(126,317)$. Whether this occurs in the myocardium is not clear. Preferential metabolism of fatty acids and reduced glucose utilization correlate with the reduced muscle ETC activity (189) and with the decreased whole-body anaerobic capacity (315). Studies demonstrated disparate effects of oxidative stress on mitochondria and implicated oxidative stress as a cause of mitochondrial dysfunction in diabetic hearts (Fig. 12).

The low levels of $\mathrm{O}_{2}{ }^{-\bullet}$ and $\mathrm{H}_{2} \mathrm{O}_{2}$ normally produced by mitochondria are overproduced in MS and associated diseases $(219,316,317)$. In fact, in MS and diabetes, ROS may be predominantly derived from mitochondria as opposed to cytosolic origin. As described above (Fig. 5), excess $\mathrm{O}_{2}{ }^{-\bullet}$ can (i) directly damage iron-sulfur center-containing enzymes, (ii) be converted to $\mathrm{H}_{2} \mathrm{O}_{2}$ (and ultimately to $\mathrm{OH}^{\bullet}$ ), and (iii) react with $\mathrm{NO}^{\bullet}$ to produce $\mathrm{ONOO}^{-}$, a potentially dangerous RNS (19). As said above, fatty acids are particularly sensitive to ROS/RNS oxidation, resulting in the formation of lipid peroxides, which are cytotoxic and lead to free-radical damage of other lipids, proteins, and DNA, especially mitochondrial DNA $(119,316)$. Even before the diagnosis of MS or type-2 diabetes, the accumulation of oxidized fatty acids in mitochondria can result in a progressive oxidative damage. In fact, 


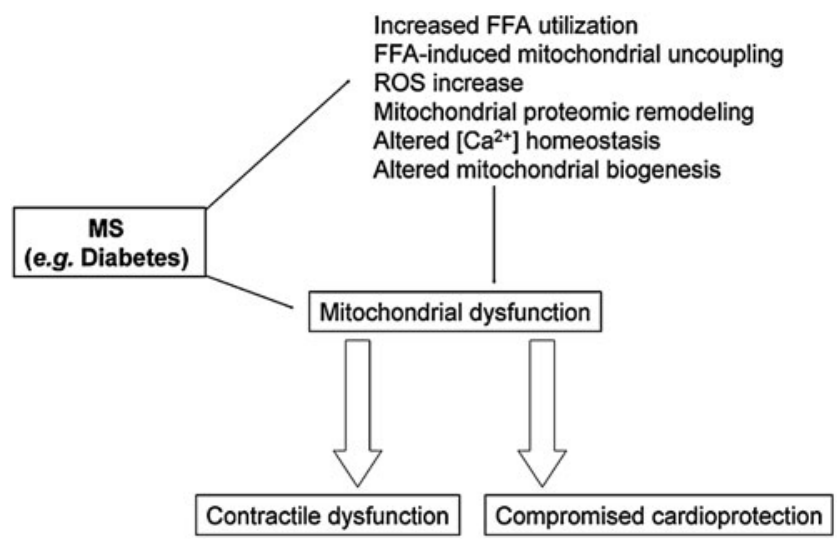

FIG. 12. Main alterations leading to mitochondrial and cardiac dysfunction in metabolic syndrome (MS). MS is considered as one of the archetypal conditions that may alter mitochondrial function, thus compromising the possibility to induce cardioprotection. For other acronyms, see the list of Abbreviations Used.

continually excessive ROS production by mitochondria has a role in the development and progression of diabetes and its complications, including cardiac pathologies.

Mitochondrial proteome changes with diabetes types 1 and $2(38,320)$. Moreover, MS and diabetes are also associated with alterations in kinase phosphorylation, in particular in ERK1/2 and Akt phosphorylation, possibly due to alteration in phosphatase activity $(164,166,293,359,368)$.

Taken together, in diabetes and MS, in general, altered mitochondrial substrate flux, increased activation of UCPs, reduced activation of kinases, and excessive ROS/RNS production may play important roles in the development and progression of the disease and in contributing to cardiac dysfunction (Fig. 12). Thus, novel treatments that are targeted to these alterations might lead to new therapeutic approaches for the prevention of cardiac dysfunction.

1. Cardioprotection in MS. In support of the importance of the oxidative stress in diabetic cardiomyopathy, Shen et al. (320) have reported that protection of both cardiac function and mitochondria can be induced by overexpression of MnSOD. These authors also reported that morphological alterations (broken mitochondrial membrane, mottled matrix, and swelling) and the increase in mitochondrial biogenesis could be observed and reversed as well. Also, AMPK inactivation has been linked to diabetes and MS in general. For instance, genetic ablation of the AMPK $\alpha 2$-catalytic subunit in murine models led to a phenotype of MS (370). Moreover, as said above, glucose metabolism during myocardial ischemia appears to be affected by impairment of AMPK in the heart $(51,53)$. Therefore, it has been proposed that modulation of AMPK activity in the diabetic heart may overcome the increased susceptibility to I/R injury (263).

In multiple models of either type 2 diabetes or MS (e.g., db / $\mathrm{db}$ mice, WOKW, Goto-Kakizaki, or Zucker-fatty rats), and in streptozotocin-induced type 1 diabetes, cardioprotection achieved with ischemic or pharmacological PreC is either absent $(123,190,203,350)$ or markedly attenuated (359). It has been also observed that an augmented stimulus may be required to achieve the threshold for a critical level of Akt phosphorylation and cardioprotection in diabetic rat hearts (359). Of note, during the initial development of experimentally induced type 1 diabetes, the heart is more resistant to I/R injury (104).

Also, ischemic PostC is ineffective in limiting I/ R injury (39, 293) and may even increase the infarct size in the presence of diabetes (284). Similar negative findings have been obtained with pharmacological PostC with anesthetics (iso- and sevoflurane) $(165,298)$. Intriguingly, direct targeting of the mPTP with CsA has been shown to restore the protective effects of sevoflurane $(165,298)$, thus suggesting that alteration in signaling associated with hyperglycemia may be mainly located to upstream components of the cardioprotective pathways. Nevertheless, a direct targeting of mPTP is not always effective to restore PostC protection (164). Another strategy that reestablished the protective effects of PostC was the normalization of blood insulin and glucose levels via islet cell transplantation (284). These findings are important, as they imply that signaling and mitochondrial defects in diabetic hearts are potentially reversible.

\section{B. Cardioprotection in Aging}

Clearly, the cardioprotective effect of PreC and PostC and pharmacological conditioning decrease with aging $(1-3,25$, 30, 292).

Oxidative stress is considered causal for the process of aging $(330,375)$. Aging cardiomyocytes are subjected to enhanced ROS production, which damages mitochondria, reduces mitochondrial fission, and contributes to the formation of larger mitochondria $(351,375)$. These giant mitochondria are not effectively removed by mitophagy, and therefore accumulate within cells. They often contain mutated DNA and altered proteins of the respiratory chain, and therefore contribute to excessive ROS formation and further oxidative protein damage (330, 351, 375).

Age and associated comorbidities are characterized by changes in the expression and activity of typical cardioprotective signaling proteins (e.g., ERK1/2, PI3K-Akt, STAT-3, p38 MAPKs, and PKCs), both under baseline conditions and in response to stressors such as I/R $(123,168,200,201,242)$. It is not yet clear if any of this variability in response originates at the mitochondrial level. However, it has been suggested that Cx43 and STAT-3 are reduced not only in ventricular total protein extracts but also in mitochondria isolated from aged mice $(28,30)$. Moreover, the amounts of nuclear encoded mitochondrial proteins are also affected by age and associated diseases. These include proteins involved in ATP synthesis and mitochondrial respiration, as well as STAT-3 and Cx43, which are considered important for cardioprotection $(24,31$, 32). In fact, with aging the transcription of genes encoding proteins with different functions, including those of ETC (23), is reduced, thus contributing to the development of mitochondrial dysfunction (27).

A decreased level of STAT-3 in aged hearts may affect the expression level of STAT-3-target genes. Indeed, the expression of MnSOD is decreased in aged rat hearts (105). However, in mice, mRNA and protein levels of the STAT-3-target iNOS are elevated with age $(14,381)$. It is likely that increased levels of TNF- $\alpha$ in aged mouse hearts may contribute to the enhanced transcription of iNOS via enhanced $\mathrm{O}_{2}{ }^{-}$formation and subsequent $\mathrm{NF} \kappa \mathrm{B}$ activation (14). Since the 
cardioprotection by SWOP and pharmacological PreC is dependent on iNOS $(35,265)$, the enhanced iNOS levels may contribute to the preservation of SWOP in aged hearts.

On the whole, in aging, there is an increased susceptibility to $I / R$, which is likely due to enhanced oxidative stress (31, 330, 351). Moreover, ischemic PreC and PostC lose their effectiveness. The mechanisms responsible for the loss of protection in the aged heart include alterations in gene/protein expression, signal transduction cascades, and altered mitochondrial function (e.g., ROS formation and respiration).

However, some contrasting results are reported; for example, a limitation of I/R injury with PreC has been reported in rabbits aged $2-4$ years $(227,291)$ and in sheep aged $5-8$ year (45); rats aged 16-18 months did not lose the possibility to protect the heart with ischemic or pharmacological PostC $(323,385)$. We cannot rule out that an inappropriate definition of aged cohorts may explain some of these discrepant findings. In fact, we should keep in mind that aging is a continuum; thus, a temporal phase of adaptability in cardioprotective signaling, including alternative mediators or signaling pathways, may explain some positive results in models of initial aging (291). In this regard, prolonged, preischemic administration of opioids, as well as caloric restriction and exercise, has been seen to contribute to adaptations that prevent the age-induced loss of cardioprotection $(1,2,256)$.

\section{Cardioprotection in hypertension and hypertrophy}

Left ventricular hypertrophy as a consequence of sustained elevation of cardiac work load is an important form of cardiac remodeling, which occurs in many pathological conditions, among which arterial hypertension is the most common. There is experimental evidence that hypertrophied myocardium is at a greater risk of sustaining injury after I/ $\mathrm{R}$ [for review see (104)]. However, there are few studies in hypertensive animals with infarct size as the principal end-point, and these studies have not clearly demonstrated an increased infarct size in the hypertrophied heart $(93,104,278)$. Nevertheless, several biochemical and metabolic alterations have been observed in the hypertrophic myocardium. These include altered mitochondrial energetics and ATP production and alterations in glycolytic metabolism with lactate accumulation during ischemia $(4,250,257)$. Moreover, increased ROS generation and reduced antioxidant potential have been described (13). Although these data have not been consistent, they may explain the increased sensitivity of hypertrophied myocardium to I/R injury (104).

The cardioprotective effect of pharmacological PreC is blunted in models of hypertension and hypertrophy. Yet from studies on ischemic PreC, it seems that the protection is conserved in the early stages of hypertrophy and lost as hypertrophy increases $(92,93,104)$. Interestingly, initial reports also suggest that the beneficial effects of PostC are largely maintained in models of hypertension and/or hypertrophy (103, $215,278,394)$. Among the studies in which the infarct size was assessed, one has demonstrated robust, PostC-induced cardioprotection in a mouse model of transverse aortic constriction via the ERK1/2 pathway (215); another study has shown that PostC protects remodeled hypertrophic myocardium via the PI3K-PKB/Akt pathway (394). We observed a nonsignificant trend toward reduction of infarct size with PostC in spontaneously hypertensive rats (278). Moreover, in this study, we have shown that protection by PostC cannot be added to the beneficial effects of antihypertensive therapy with captopril. In other studies $(267,279)$, we have shown that during nandrolone treatment, which induces a progressive myocardial hypertrophy, the efficacy of ischemic PostC was preserved or even enhanced in a not-yet hypertrophic heart; this is reminiscent of the supernormal period (with increased resistance to I/R) observed during experimental diabetes development. However, the protective efficacy of PostC was lost in hearts clearly hypertrophic. In this study, we have shown that the levels of survival kinases change during hypertrophy development (279).

Whether infarct size reduction with ischemic PreC and PostC is, indeed, preserved in hypertension and/or hypertrophic myocardium remains to be substantiated in future studies. Moreover, the possible alterations of the cardioprotective pathways, especially those of PostC, in the hypertrophic/hypertensive heart need to be further studied.

\section{Transition to the Clinical Setting}

PreC with ischemia (including remote PreC) or drugs may be applied in planned interventions in the clinical arena. However, PreC is not always feasible, because the occurrence of AMI is hardly predictable. Instead, ischemic PostC appears to be feasible in the majority of patients, including STEMI patients with AMI. PostC, that is, brief episodes of ischemia and reperfusion can be performed at the time of reflow during $\mathrm{PCI}$ or direct stenting. In fact, the PostC stimulus applied in such a patient with AMI using inflation and deflation of angioplasty balloon after reopening of coronary artery reduced the infarct size $(333,356)$. Therefore, PostC transition to the clinical setting has proven the existence of lethal myocardial reperfusion injury in man, and the clinical studies suggest that $40 \%-50 \%$ of the final reperfused infarct in humans may be attributable to myocardial reperfusion injury (384). In an original study, the investigators evaluated the level of oxidative stress associated with reperfusion in patients undergoing PCI and ischemic PostC (225). Malondialdehyde, a nonspecific marker of lipid peroxidation, was measured in blood several times during the first 7 days of reperfusion. The level of malondialdehyde was significantly lower in the postconditioned than in the control patients, suggesting that a part of the beneficial effect of PostC could be explained by a reduction in oxidative stress (225), as shown in experimental studies $(339,393)$. Moreover, PostC has been effectively used in several settings, including before removal of aortic crossclamping after cardiac surgery (223).

However, two recent studies $(111,332)$ using the established ischemic PostC algorithm of four cycles of 1-min reocclusion/reperfusion found no protection in terms of either creatin kinase and troponin release or infarct size measurement by magnetic resonance imaging. Therefore, in humans as well as in experimental animals, there are both several positive and a few negative studies with ischemic PostC (154, 327).

\section{A. The possible reasons of variable outcomes with ischemic PostC}

In our opinion, it is not surprising that a variability (ranging from highly protective to negative results) in the magnitude of myocardial salvage can be observed among some clinical and 
experimental studies. Both the $I / R$ injury and the PostC protection may be influenced by a plethora of conditions that is not easy to keep under the control of the operators (physicians, cardiologists, and researchers), especially in the clinical arena. Particularly relevant are the variables correlated to artery reopening. For example, in determining whether I/R injury may be relevant whether or not the artery is fully reopened. This is also relevant in determining PostC cardioprotection: an artery not completely reopened in the I/R setting (control group) may exert a sort of gentle reperfusion that may reduce the infarct size, thus restricting the difference with hearts protected by ischemic PostC (stuttering reperfusion in the treatment group). On the other hand, an artery not completely reopened during Post $C$ maneuvers may reduce cardioprotective effects, thus further restricting the difference with hearts treated with full reperfusion only. Many of the negative studies with PostC did not check whether or not the artery was fully reopened during the stuttering reperfusion (PostC maneuvers). Moreover, as said above, factors that are known to influence/limit the cardioprotective benefits of both PreC and PostC include age, gender, and the use of drugs (e.g., $46,161,235,277$, and 350). In fact, a number of medications can limit I/R injury, including ACE inhibitors, adenosine, AT1-blockers, statins, $\beta$-blockers, dronedarone, ivabradine, and nitroglycerine (60, 149, 150, 272, 278, 327). Moreover, drugs such as COX inhibitors can interfere with PostC molecular mechanisms (273). Therefore, it is not only the attenuation of protection in the PostC cohort but also the potential recruitment of protection in the control group, which may minimize any difference between PostC and control. Moreover, another possible confounder is the presence of comorbidities (MS, diabetes and hyperlipidemia, and hypertension), including increased age, which are all risk factors for cardiovascular diseases (see above). In fact, patients exhibiting one or more of these risk factors are the actual populations in which the incidence of AMI is greatest, and thus cardioprotection is required $(104,221)$.

Therefore, due to the complexity of alterations in the presence of comorbidities and due to complexity and variability of ischemic PostC responses, this procedure will be translated to a clinical routine only when the signaling pathway is fully understood. Moreover, we need to understand the reasons for the incongruity between experimental models and clinical outcome, especially in the settings with comorbidities. In fact, it seems that the majority of comorbidities limit cardioprotection in the experimental setting, whereas comorbidities do not appear as a major problem in a clinical setting $(179,284,333,353)$. How can this discrepancy be reconciled? One difference may be due to the fact that humans with comorbidities are receiving adequate treatments. There are encouraging clinical data suggesting that patients with AMI may benefit from preserved mitochondrial function, thereby emphasizing the paramount importance of mitochondria as therapeutic targets to limit I/R injury. The beneficial effects are obtained despite an important incidence of comorbidities in these patients.

\section{B. Pharmacological PostC}

Since $\mathrm{MPTP}$ is a major factor in determining cell death and is considered one final step in cardioprotective signaling cascade, mPTP inhibition affords significant cardioprotection
$(10,81,127,142)$; in fact, this concept has been successfully translated into clinical practice (e.g., 179 and 284). Yet, ischemic PostC may be limited to angioplasty and surgery, and may be associated with increased complications of the operative procedure, and thus the most constructive strategy would be pharmacological PostC.

Pharmacological PostC would avoid the unfavorable consequences linked with intermittent artery cross-clamping and provide a simple method of myocardial protection subsequent to all cardiac procedures, including coronary reopening with thrombolytics (384). However, caution must be used; in fact, previous attempts to intervene at the onset of reperfusion using pharmacological agents as a strategy for protecting against myocardial reperfusion injury have been extensively investigated, and in terms of translation, the treatment strategy into clinical therapy has been largely disappointing (33, 384). For instance, adenosine has been considered for long time as the ideal candidate for protection against I/R damage, based on several experimental evidence of its protective effects, which however did not solve the conundrum of effective receptors: adenosine has at least four receptors that can change their affinity for the agonist at different times of ischemia and reperfusion, and at present, it is unclear which ones of the different receptors are more important at different stages $(60,61,368)$. Despite intensive investigation with adenosine and specific receptor agonists, it is difficult to conclude as to a protective role of adenosine in STEMI patients $(179,303)$. Glucagon-like peptide-1 is another drug that has been demonstrated to improve the left ventricular ejection fraction by about $35 \%$ in patients with severely reduced left ventricular function after AMI (245), but the study was not designed to reduce lethal reperfusion injury, as the drug was administered several hours after reperfusion, that is, far from the therapeutic window for reperfusion injury. However, initial progress has been made with more novel approaches for preventing myocardial reperfusion injury administering the drug in the first minutes of reperfusion (135). As said, preliminary clinical data indicate that drugs targeting $\mathrm{mPTP}$ or RISKs may confer benefits to patients with AMI, with and without comorbidities, increasing that provided by myocardial reperfusion alone. Very good results are obtained with drugs such as CsA as an mPTP desensitizer $(230,284,333)$, as well as with other drugs targeting RISKs, such as erythropoietin and its analogs (e.g., 22 and 44). Moreover, the protective effects of the $\mathrm{K}_{\text {ATP }}$ channel opener, nicorandil, on the one hand, and natriuretic peptide, on the other hand, were studied in J-WIND trials (195). Treatment with a natriuretic peptide was associated with a small but significant reduction in the infarct size and improvement in the ejection fraction, corresponding to a better clinical outcome. In this study, nicorandil did not affect the size of infarct size, though oral administration of nicorandil during follow-up increased the left ventricular ejection. In another small study, a dose of nicorandil that was three times higher than that used in J-WIND decreased the infarct size and reduced re-admission to hospital for chronic heart failure or the rate of cardiovascular death in patients with AMI (178).

The treatment with CsA in the setting of AMI in conjunction with reperfusion therapy was safe and significantly reduced the size of the infarction (179). In a follow-up study, Mewton et al. (230) recently examined whether CsA might have modified LV remodeling in that subgroup of patients 
with appreciable effects on the infarct size. The authors described that a reduction persisted after 6 months, and that CsA did not exert any deleterious effect on LV remodeling.

Importantly, similar cardioprotective effects were obtained with other drugs acting on $\mathrm{mPTP}$, confirming the relevance of this approach. For instance, derivatives of CsA, such as [Nmethyl-ala ${ }^{6}$ CsA, [N-methyl-Val ${ }^{4}$ ]CsA, Debio 025, NIM811, or sanglifehrin A (126-128, 133, 134, 136-139, 141, 142, 230), also prevent myocardial I/ $R$ injury in an experimental setting. TRO40303 is a new drug that inhibits mPTP triggered by oxidative stress. Its efficacy in an animal model of AMI makes TRO40303 a promising new drug for the reduction of myocardial I/R injury, which acts on mitochondrial translocator protein $18 \mathrm{kDa}$ (TSPO) at the cholesterol site. TSPO is a fivetransmembrane-domain protein that is localized primarily in the OMM, and it is involved in the translocation of cholesterol from the OMM to the IMM. Since TRO40303 binds to the TSPO, its mode of action differs from that of other mPTP desensitizer, such as CsA, thus providing a new promising pharmacological approach to study $\mathrm{mPTP}$ regulation (313). In our opinion, a fortunate aspect of these drugs is that they are not inhibitors, but desensitizers, which do not abolish ROS production in any cellular conditions, but only inhibits it, thus, might not interfere with the protective redox signaling.

One molecule that has received much attention is nitrite $\left(\mathrm{NO}_{2}{ }^{-}\right)$(324). A strong debate surrounds the mechanisms of nitrite-mediated cardioprotection, with a central focus on the identification of the nitrite reductase responsible for the biotransformation of this molecule in hypoxia. Candidates for this activity include deoxyhemoglobin, deoxymyoglobin, and various components of the respiratory chain itself. In addition, the mechanism by which the hypoxic metabolites of nitrite bring about protected phenotype is also matter of controversy, but interestingly, it is thought that $\mathrm{MPTP}$, ROSmodulation, and SNO of proteins may play a role $(306,309)$. Notably, regardless of these mechanisms, nitrite is now entering clinical trials for AMI. Much attention in the cardioprotection field has also focused on the effects of volatile anesthetics, which are largely shown to be cardioprotective in many species, including humans $(130,165,214,298,336)$. It has been shown that some of these anesthetics can inhibit mitochondrial respiration, whereas others are thought to activate $\mathrm{K}^{+}$channels in the mitochondrial membrane $(214,336)$. Yet, irrespective of the exact mechanism, anesthetics currently represent one of the most widely used, safest, and most clinically applicable cardioprotective agents.

\section{Features of a successful cardioprotective approach in early reperfusion}

Due to the abundance of mitochondria in cardiomyocytes and to the compelling dependence of myocardial function on oxidative phosphorylation, it is hardly surprising that treatments targeting these organelles are successful in myocardial cardioprotective interventions. Besides the more appropriate target (mitochondrial elements) reached by the drugs, it is likely that novel approaches can achieve more success, because the essential factors are understood and considered recently. First of all, the time window for ischemic PostC is a critical factor $(193,269,270)$. In fact, during angioplasty, the treatment (either ischemic or pharmacological PostC) should be applied before (drugs) or within the first minute after direct stenting of the culprit coronary artery. Several other major confounding factors that have been shown to deeply influence the results of all infarct size reduction studies must be taken into consideration, including the timing of the protective intervention with respect to reflow and the major determinants of infarct size (duration of ischemia, size of the area at risk, collateral circulation, and microembolic complications) (147). Besides the phosphorylation, also the redox signaling, the SNO and the other forms of signaling in the early reperfusion should be borne in mind for a successful treatment in reperfusion. The disappointing results with antioxidants may be due, at least in part, to limitation of protective ROS in the very early phase of reperfusion (260). The possibility also exists that intermittent treatment might be helpful (271-273). In fact, we have shown that brief repetitive (intermittent) administration of bradykinin during early reperfusion can trigger PostC through a redox signaling (271). Finally, Cohen et al. (64) have highlighted the fact that staccato reperfusion reintroduces intermittent reoxygenation to perpetuate myocardial acidosis and to initiate protection.

Therefore, a pharmacological approach in the very early phase of reperfusion, which targets mitochondria, allows a ROS signaling, and preserves acidosis, may represent an important goal for strategies protecting ischemic-reperfused myocardium. Of course, the presence of comorbidities and drugs already used by the patient must be considered and targets adjusted accordingly.

\section{Executive Summary and Conclusions}

It appears that many different signals can target mitochondria and can induce $\mathrm{mPTP}$ formation, strictly linking I/R stress and damage to these organelles. This emphasizes the capacity of mitochondria to function as general cell-death sensors and to integrate many lethal signals.

ROS production increases during ischemia until the $\mathrm{O}_{2}$ is exhausted; then, it may further increase in early reperfusion $(17,89,191,220,396,401)$. In ischemic hearts, the $\mathrm{pH}$ falls to 6.8 or less, but abruptly rises with reperfusion. Recovery of $\mathrm{pH}$ is due to the action of membrane exchangers and to $\mathrm{H}^{+}$ washout of the previously ischemic cells by restored blood flow. Formation of $\mathrm{MPTP}$ is limited during ischemia by the low $\mathrm{pH}$ despite increased cellular levels of ROS and $\mathrm{Ca}^{2+}$ (Fig. 1A). However, as $\mathrm{pH}$ returns to its baseline level and ROS formation increases, $\mathrm{mPTP}$ opens; therefore, ROS production is hastened by RIRR; mitochondrial electrochemical gradients are disrupted; ATP production is halted; and the cell is targeted for apoptosis and necrosis (Fig. 1B).

In the protected hearts, slightly/slow $\mathrm{pH}$ changes during reperfusion are suggested. In particular, elaborate signaling during the brief PreC and PostC ischemia is triggered, including small ROS formation with an important redoxsignaling role (Fig. 3). In reperfusion, as soon as flow and $\mathrm{O}_{2}$ are restored to the myocardium, a huge production of deleterious ROS may occur. However, slight production of ROS and a slower recovery of $\mathrm{pH}$ may occur in preconditioned hearts, which together with other endogenous protagonists lead to prevention of $\mathrm{mPTP}$ opening. In postconditioned hearts, the repeated brief coronary reocclusions keep the $\mathrm{pH}$ low enough to inhibit MPTP opening at the onset of reperfusion. In the meantime, the intermittent reoxygenation permits only a low amount of ROS with an important signaling role. 
This provides time for the signaling to generate those endogenous inhibitors of mPTP formation that keep the pores from opening even after return of $\mathrm{pH}$ to the baseline value. Yet, after a triggering phase in the preischemic period, the actual protection by PreC occurs in the reperfusion rather than in the ischemic phase with the repopulation of sensitized GPCR at the beginning of myocardial reperfusion after the index ischemia (i.e., a possible sensitization of relevant receptors is induced by prior ischemias). GPCR agonists include adenosine, bradykinin, and opioids. The exact subtypes of adenosine, opioids, and bradykinin receptors involved in triggering protection are still a matter of controversy. Receptor tyrosine kinases may be also involved, possibly via the EGF receptor transactivation to activate PI3K/Akt, so that after release/accumulation of ligand(s), GPCR-respective receptor engagement leads to activation of both ERK1/2, and to activation of the PI3K/Akt cassette. Both PreC and PostC engage GPCR and enzymes (Akt, AMPK, PKC, GSK-3 $\beta$, etc.), which converge on the $\mathrm{mPTP}$. Importantly, PKC can be activated by (i) Akt signaling, (ii) phospholipase signaling, (iii) ROS signaling, and (iv) cGMP/PKG pathway. The latter may start from activated Akt, which can phosphorylate multiple substrates, including eNOS, which activate the PKG path via elevated $\mathrm{NO}^{\bullet} / \mathrm{cGMP}$, both in PreC and PostC. Thus, both PreC and PostC activate cardioprotective pathways that are protective against reperfusion injury. Another prosurvival factor activated in response to TNF receptors binding is the JAK/STAT-3 pathway (Fig. 9). A cross-talk between this pathway and the Akt pathway may exist. All these signal transsduction pathways then terminate on mitochondria, regulating mitochondrial function and keeping $\mathrm{mPTP}$ close. As said, the closure of the MPTP is also due to the acidosis, in the early stages of reperfusion. Autophagy and mitophagy are other mechanisms that may influence the outcome of cardioprotection.

Several proof-of-concept clinical studies on ischemic and pharmacological PostC tested ischemic PostC at the beginning of angioplasty or direct stenting, as well as pharmacological PostC. Indeed, some of these studies demonstrated beneficial effects of the MPTP desensitizer CsA during early reperfusion in patients with AMI $(179,230,333)$. These are relatively safe and relatively easy-to-perform procedures, and might induce a persistent reduction in the infarct size and improvement in myocardial contractile function $(333,356)$. However, a couple of small clinical studies have obtained negative results with PostC (150). Nevertheless, the question of whether these treatments improve clinical outcomes has not been assessed yet in clinical trials, including a large-scale, multicenter, controlled, randomized study with multiple patients' stratification. Such studies to determine the impact of ischemic and pharmacological PostC on patient outcomes are mandatory. Moreover, since mitochondria and ROS are attractive mechanistic targets for cardioprotection, much attention should be devoted to the critical points above considered (confounding comorbidities, co-medications, problems with interventional procedures and artery reopening, timing of the interventions, redox signaling, etc.). Personalized therapies directed to either prevent mPTP formation or the events leading to MPTP opening might be achievable in the future. Although we have made enormous progresses in the treatment of cardiac ischemia, there remains a need for innovative treatments to further decrease the morbidity and mortality. In the AMI patients, a pharmacological approach in the very early phase of reperfusion that (i) targets MPTP, (ii) allows a ROS signaling, and (iii) preserves early acidosis needs to be tested.

\section{Acknowledgments}

The work of authors is funded by National Institutes of Cardiovascular Research, Regione Piemonte, PRIN, ex- $60 \%$ and Compagnia di San Paolo. We thank Prof. Donatella Gattullo for her invaluable support.

\section{References}

1. Abete P, Cacciatore F, Testa G, Della-Morte D, Galizia G, de Santis D, Calabrese C, Cioppa A, Ferrara N, and Rengo F. Ischemic preconditioning in the aging heart: from bench to bedside. Ageing Res Rev 9: 153-162, 2010.

2. Abete $P$, Calabrese C, Ferrara N, Cioppa A, Pisanelli P, Cacciatore F, Longobardi G, Napoli C, and Rengo F. Exercise training restores ischemic preconditioning in the aging heart. J Am Coll Cardiol 36: 643-650, 2000.

3. Abete P, Testa G, Ferrara N, De Santis D, Capaccio P, Viati L, Calabrese C, Cacciatore F, Longobardi G, Condorelli M, Napoli C, and Rengo F. Cardioprotective effect of ischemic preconditioning is preserved in food-restricted senescent rats. Am J Physiol Heart Circ Physiol 282: H1978-H1987, 2002.

4. Anderson PG, Allard MF, Thomas GD, Bishop SP, and Digerness SB. Increased ischemic injury but decreased hypoxic injury in hypertrophied rat hearts. Circ Res 67: 948959, 1990.

5. Argaud L, Gateau-Roesch O, Augeul L, Couture-Lepetit E, Loufouat J, Gomez L, Robert D, and Ovize M. Increased mitochondrial calcium coexists with decreased reperfusion injury in postconditioned (but not preconditioned) hearts. Am J Physiol Heart Circ Physiol 294: H386-H391, 2008.

6. Argaud L, Gateau-Roesch O, Raisky O, Loufouat J, Robert $\mathrm{D}$, and Ovize M. Postconditioning inhibits mitochondrial permeability transition. Circulation 111: 194-117, 2005.

7. Baines CP, Kaiser RA, Purcell NH, Blair NS, Osinska H, Hambleton MA, Brunskill EW, Sayen MR, Gottlieb RA, Dorn GW, Robbins J, and Molkentin JD. Loss of cyclophilin $\mathrm{D}$ reveals a critical role for mitochondrial permeability transition in cell death. Nature 434: 658-662, 2005.

8. Baines CP, Kaiser RA, Sheiko T, Craigen WJ, and Molkentin JD. Voltage dependent anion channels are dispensable for mitochondrial-dependent cell death. Nat Cell Biol 9: 550-555, 2007.

9. Baines CP, Zhang J, Wang G-W, Zheng Y-T, Xiu JX, Cardwell EM, Bolli R, and Ping P. Mitochondrial PKC $\varepsilon$ and MAPK form signaling modules in the murine heart. Circ Res 90: 390-397, 2002.

10. Baines $\mathrm{CP}$. The mitochondrial permeability transition pore and ischemia/reperfusion injury. Basic Res Cardiol 104: 181188, 2009.

11. Barger PM and Kelly DP. PPAR signaling in the control of cardiac energy metabolism. Trends Cardiovasc Med 10: 238245, 2000.

12. Basso E, Petronilli V, Forte MA, and Bernardi P. Phosphate is essential for inhibition of the mitochondrial permeability transition pore by cyclosporin A and by cyclophilin D ablation. J Biol Chem 283: 26307-26311, 2008.

13. Batist G, Mersereau W, Malashenko BA, and Chiu RC. Response to ischemia-reperfusion injury in hypertrophic 
heart. Role of free-radical metabolic pathways. Circulation 80: III10-13, 1989.

14. Bátkai S, Rajesh M, Mukhopadhyay P, Haskó G, Liaudet L, Cravatt BF, Csiszár A, Ungvári Z, and Pacher P. Decreased age-related cardiac dysfunction, myocardial nitrative stress, inflammatory gene expression, and apoptosis in mice lacking fatty acid amide hydrolase. Am J Physiol Heart Circ Physiol 293: H909-H918, 2007.

15. Baur JA, Pearson KJ, Price NL, Jamieson HA, Lerin C, Kalra A, Prabhu VV, Allard JS, Lopez-Lluch G, Lewis K, Pistell PJ, Poosala S, Becker KG, Boss O, Gwinn D, Wang M, Ramaswamy S, Fishbein KW, Spencer RG, Lakatta EG, Le Couteur D, Shaw RJ, Navas P, Puigserver P, Ingram DK, de Cabo R, and Sinclair DA. Resveratrol improves health and survival of mice on a high-calorie diet. Nature 444: 337-342, 2006.

16. Beauloye C, Bertrand L, Horman S, and Hue L. AMPK activation, a preventive therapeutic target in the transition from cardiac injury to heart failure. Cardiovasc Res 90: 224233, 2011.

17. Becker LB, vanden Hoek TL, Shao Z-H, Li C-Q, and Schumacker PT. Generation of superoxide in cardiomyocytes during ischemia before reperfusion. Am J Physiol Heart Circ Physiol 277: H2240-H2246, 1999.

18. Beckman JS and Koppenol WH. Nitric oxide, superoxide, and peroxynitrite: the good, the bad, and ugly. Am J Physiol 271: C1424-C1437, 1996.

19. Beckman JS, Beckman TW, Chen J, Marshall PA, and Freeman BA. Apparent hydroxyl radical production by peroxynitrite: implications for endothelial injury from nitric oxide and superoxide. Proc Natl Acad Sci U S A 87: 16201624, 1990.

20. Beltran B, Mathur A, Duchen MR, Erusalimsky, JD, and Moncada S. The effect of nitric oxide on cell respiration: a key to understanding its role in cell survival or death. Proc Natl Acad Sci U S A 97: 14602-14607, 2000.

21. Bernardi P, Krauskopf A, Basso E, Petronilli V, BlalchyDyson E, Di Lisa F, and Forte MA. The mitochondrial permeability transition from in vitro artifact to disease target. FEBS J 273: 2077-2099, 2006.

22. Binbrek AS, Rao NS, Al Khaja N, Assaqqaf J, and Sobel BE. Erythropoietin to augment myocardial salvage induced by coronary thrombolysis in patients with ST segment elevation acute myocardial infarction. Am J Cardiol 104: 10351040, 2009.

23. Bodyak N, Kang PM, Hiromura M, Sulijoadikusumo I, Horikoshi N, Khrapko K, and Usheva A. Gene expression profiling of the aging mouse cardiac myocytes. Nucleic Acids Res 30: 3788-3794, 2002.

24. Bodyak N, Rigor DL, Chen YS, Han Y, Bisping E, Pu WT, and Kang PM. Uncoupling protein 2 modulates cell viability in adult rat cardiomyocytes. Am J Physiol Heart Circ Physiol 293: H829-H835, 2007.

25. Boengler K, Buechert A, Heinen Y, Roeskes C, HilfikerKleiner D, Heusch G, and Schulz R. Cardioprotection by ischemic postconditioning is lost in aged and STAT3-deficient mice. Circ Res 102: 131-135, 2008.

26. Boengler K, Dodoni G, Ruiz-Meana M, Cabestrero A, Rodriguez-Sinovas A, Garcia-Dorado D, Gres P, Di Lisa F, Heusch G, and Schulz R. Connexin 43 in cardiomyocyte mitochondria and its increase by ischemic preconditioning. Cardiovasc Res 67: 234-244, 2005.

27. Boengler K, Heusch G, and Schulz R. Mitochondria in postconditioning. Antioxid Redox Signal 14: 863-880, 2011.
28. Boengler K, Hilfiker-Kleiner D, Drexler H, Heusch G, and Schulz R. The myocardial JAK/STAT pathway: from protection to failure. Pharmacol Ther 120: 172-185, 2008.

29. Boengler K, Hilfiker-Kleiner D, Heusch G, and Schulz R. Inhibition of permeability transition pore opening by mitochondrial STAT3 and its role in myocardial ischemia/ reperfusion. Basic Res Cardiol 105: 771-785, 2010.

30. Boengler K, Konietzka I, Buechert A, Heinen Y, GarciaDorado D, Heusch G, and Schulz R. Loss of ischemic preconditioning's cardioprotection in aged mouse hearts is associated with reduced gap junctional and mitochondrial levels of connexin 43. Am J Physiol Heart Circ Physiol 292: H1764-H1769, 2007.

31. Boengler K, Schulz R, and Heusch G. Loss of cardioprotection with ageing. Cardiovasc Res 83: 247-261, 2009.

32. Boengler K, Stahlhofen S, van de Sand A, Gres P, RuizMeana M, Garcia-Dorado D, Heusch G, and Schulz R. Presence of connexin 43 in subsarcolemmal, but not in interfibrillar cardiomyocyte mitochondria. Basic Res Cardiol 104: 141-147, 2009.

33. Bolli R, Becker L, Gross G, Mentzer R, Jr., Balshaw D, and Lathrop DA. Myocardial protection at a crossroads: the need for translation into clinical therapy. Circ Res 95: 125-134, 2004.

34. Bolli R, Dawn B, and Xuan Y-T. Role of the JAK-STAT pathway in protection against myocardial ischemia/reperfusion injury. Trends Cardiovasc Med 13: 72-79, 2003.

35. Bolli R, Li QH, Tang XL, Guo Y, Xuan YT, Rokosh G, and Dawn $\mathrm{B}$. The late phase of preconditioning and its natural clinical application-gene therapy. Heart Fail Rev 12: 189199, 2007.

36. Bolli R, Stein AB, Guo Y, Wang OL, Rokosh G, Dawn B, Molkentin JD, Sanganalmath SK, Zhu Y, and Xuan YT. A murine model of inducible, cardiac-specific deletion of STAT3: its use to determine the role of STAT3 in the upregulation of cardioprotective proteins by ischemic preconditioning. J Mol Cell Cardiol 50: 589-597, 2011.

37. Bolli R. Preconditioning: a paradigm shift in the biology of myocardial ischemia. Am J Physiol Heart Circ Physiol 292: H19-H27, 2007.

38. Boudina S, Sena $S$, Theobald $H$, Sheng XM, Wright JJ, Hu XX, Aziz S, Johnson JI, Bugger H, Zaha VG, and Abel ED. Mitochondrial energetics in the heart in obesity-related diabetes-direct evidence for increased uncoupled respiration and activation of uncoupling proteins. Diabetes 56: 2457-2466, 2007.

39. Bouhidel O, Pons S, Souktani R, Zini R, Berdeaux A, and Ghaleh B. Myocardial ischemic postconditioning against ischemia-reperfusion is impaired in ob/ob mice. Am J Physiol Heart Circ Physiol 295: H1580-H1586, 2008.

40. Brand MD and Esteves TC. Physiological functions of the mitochondrial uncoupling proteins UCP2 and UCP3. Cell Metab 2: 85-93, 2005.

41. Brennan JP, Southworth R, Medina RA, Davidson SM, Duchen MR, and Shattock MJ. Mitochondrial uncoupling, with low concentration FCCP, induces ROS-dependent cardioprotection independent of $\mathrm{K}_{\mathrm{ATP}}$ channel activation. Cardiovasc Res 72: 313-321, 2006.

42. Budas GR, Churchill EN, Disatnik M-H, Sun L, and Mochly-Rosen D. Mitochondrial import of $\mathrm{PKC} \varepsilon$ is mediated by HSP90: a role in cardioprotection from ischaemia and reperfusion injury. Cardiovasc Res 88: 83-92, 2010.

43. Bugger $\mathrm{H}$ and Abel ED. Molecular mechanisms for myocardial mitochondrial dysfunction in the metabolic syndrome. Clin Sci (Lond) 114: 195-210, 2008. 
44. Bullard AJ, Govewalla P, and Yellon DM. Erythropoietin protects the myocardium against reperfusion injury in vitro and in vivo. Basic Res Cardiol 100: 397-403, 2005.

45. Burns PG, Krunkenkamp IB, Calderone CA, Kirvaitis RJ, Gaudette GR, and Levitsky S. Is the preconditioning response conserved in senescent myocardium? Ann Thorac Surg 61: 925-929, 1996.

46. Burwell LS, Nadtochiy SM, and Brookes PS. Cardioprotection by metabolic shut-down and gradual wake-up. $J$ Mol Cell Cardiol 46: 804-810, 2009.

47. Burwell LS, Nadtochiy SM, Tompkins AJ, Young S, and Brookes PS. Direct evidence for S-nitrosylation of mitochondrial complex I. Biochem J 394: 627-634, 2006.

48. Butow RA and Avadhani NG. Mitochondrial signaling: the retrograde response. Mol Cell 14: 1-15, 2004.

49. Camara AK, Aldakkak M, Heisner JS, Rhodes SS, Riess ML, An J, Heinen A, and Stowe DF. ROS scavenging before 27 degrees $\mathrm{C}$ ischemia protects hearts and reduces mitochondrial ROS, $\mathrm{Ca}^{2+}$ overload, and changes in redox state. Am J Physiol Cell Physiol 292: C2021-C2031, 2007.

50. Camara AK, Lesnefsky EJ, and Stowe DF. Potential therapeutic benefits of strategies directed to mitochondria. Antioxid Redox Signal 13: 279-347, 2010.

51. Ceolotto G, Gallo A, Papparella I, Franco L, Murphy E, Iori E, Pagnin E, Fadini GP, Albiero M, Semplicini A, and Avogaro A. Rosiglitazone reduces glucose-induced oxidative stress mediated by $\mathrm{NAD}(\mathrm{P}) \mathrm{H}$ oxidase via AMPKdependent mechanism. Arterioscler Thromb Vasc Biol 27: 2627-2633, 2007.

52. Chacinska A, Koehler CM, Milenkovic D, Lithgow T, and Pfanner N. Importing mitochondrial proteins: machineries and mechanisms. Cell 138: 628-644, 2009.

53. Chen ZP, Mitchelhill KI, Michell BJ, Stapleton D, Rodriguez-Crespo I, Witters LA, Power DA, Ortiz de Montellano PR, and Kemp BE. AMP-activated protein kinase phosphorylation of endothelial NO synthase. FEBS Lett 443: 285-289, 1999.

54. Chou CJ, Cha MC, Jung DW, Boozer CN, Hashim SA, and Pi-Sunyer FX. High-fat diet feeding elevates skeletal muscle uncoupling protein 3 levels but not its activity in rats. Obes Res 9: 313-319, 2001.

55. Churchill EN and Mochly-Rosen D. The roles of PKCdelta and epsilon isoenzymes in the regulation of myocardial ischaemia/reperfusion injury. Biochem Soc Trans 35: 10401042, 2007.

56. Churchill EN, Disatnik MH, and Mochly-Rosen D. Timedependent and ethanol induced cardiac protection from ischemia mediated by mitochondrial translocation of variant $\mathrm{PKC} \varepsilon$ and activation of aldehyde dehydrogenase 2. J Mol Cell Cardiol 46: 278-284, 2009.

57. Churchill EN, Ferreira JC, Brum PC, Szweda LI, and Mochly-Rosen D. Ischaemic preconditioning improves proteasomal activity and increases the degradation of deltaPKC during reperfusion. Cardiovasc Res 85: 385-394, 2010.

58. Churchill EN, Murriel CL, Chen CH, Mochly-Rosen D, and Szweda LI. Reperfusion-induced translocation of deltaPKC to cardiac mitochondria prevents pyruvate dehydrogenase reactivation. Circ Res 97: 78-85, 2005.

59. Clarke SJ, Khaliulin I, Das M, Parker JE, Heesom KJ, and Halestrap AP. Inhibition of mitochondrial permeability transition pore opening by ischemic preconditioning is probably mediated by reduction of oxidative stress rather than mitochondrial protein phosphorylation. Circ Res 102: 1082-1090, 2008.
60. Cohen MV and Downey JM. Adenosine: trigger and mediator of cardioprotection. Basic Res Cardiol 103: 203-215, 2008.

61. Cohen MV and Downey JM. Ischemic postconditioning: from receptor to end-effector. Antioxid Redox Signal 14: 821831, 2011.

62. Cohen MV, Yang X-M, and Downey JM. Acidosis, oxygen, and interference with mitochondrial permeability transition pore formation in the early minutes of reperfusion are critical to postconditioning's success. Basic Res Cardiol 103: 464-471, 2008.

63. Cohen MV, Yang XM, and Downey JM. Nitric oxide is a preconditioning mimetic and cardioprotectant and is the basis of many available infarct-sparing strategies. Cardiovasc Res 70: 231-229, 2006.

64. Cohen MV, Yang X-M, and Downey JM. The $\mathrm{pH}$ hypothesis of postconditioning: staccato reperfusion reintroduces oxygen and perpetuates myocardial acidosis. Circulation 115: 1895-1903, 2007.

65. Correa F, García N, Robles C, Martínez-Abundis E, and Zazueta C. Relationship between oxidative stress and mitochondrial function in the post-conditioned heart. J Bioenerg Biomembr 40: 599-606, 2008.

66. Costa $\mathrm{AD}$ and Garlid KD. Intramitochondrial signalinginteractions among mitoK $\mathrm{ATP}_{\mathrm{ATC}} \mathrm{PK}, \mathrm{ROS}$, and MPT. Am J Physiol Heart Circ Physiol 295: H874-H882, 2008.

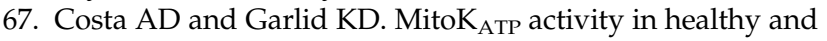
ischemic hearts. J Bioenerg Biomembr 41: 123-126, 2009.

68. Costa AD, Pierre SV, Cohen MV, Downey JM, and Garlid KD. cGMP signalling in pre- and post-conditioning: the role of mitochondria. Cardiovasc Res 77: 344-352, 2008.

69. Crisafulli A, Melis F, Tocco F, Santoboni UM, Lai C, Angioy G, Lorrai L, Pittau G, Concu A, and Pagliaro P. Exerciseinduced and nitroglycerin-induced myocardial preconditioning improves hemodynamics in patients with angina. Am J Physiol Heart Circ Physiol 287: H235-H242, 2004.

70. Crompton $\mathrm{M}$. The mitochondrial permeability transition pore and its role in cell death. Biochem J 341: 233-249, 1999.

71. Csont T, Szilvássy Z, Fülöp F, Nedeianu S, Páli T, Tosaki A, Dux L, and Ferdinandy P. Direct myocardial anti-ischaemic effect of GTN in both nitrate-tolerant and nontolerant rats: a cyclic GMP-independent activation of KATP. Br J Pharmacol 128: 1427-1434, 1999.

72. Daiber A. Redox signaling (cross-talk) from and to mitochondria involves mitochondrial pores and reactive oxygen species. Biochim Biophys Acta 1797: 897-906, 2010.

73. Das A, Xi L, and Kukreja RC. Phosphodiesterase- 5 inhibitor sildenafil preconditions adult cardiac myocytes against necrosis and apoptosis. Essential role of nitric oxide signaling. J Biol Chem 280: 12944-12955, 2005.

74. Das M, Parker JE, and Halestrap AP. Matrix volume measurements challenge the existence of diazoxide/glibencamide-sensitive $\mathrm{K}_{\mathrm{ATP}}$ channels in rat mitochondria. $J$ Physiol 547: 893-902, 2003.

75. Das S, Wong R, Rajapakse N, Murphy E, and Steenbergen C. Glycogen synthase kinase 3 inhibition slows mitochondrial adenine nucleotide transport and regulates voltagedependent anion channel phosphorylation. Circ Res 103: 983-991, 2008.

76. de Brito OM and Scorrano L. Mitofusin 2 tethers endoplasmic reticulum to mitochondria. Nature 456: 605-610, 2008.

77. Depre C, Park JY, Shen YT, Zhao X, Qiu H, Yan L, Tian B, Vatner SF, and Vatner DE. Molecular mechanisms medi- 
ating preconditioning following chronic ischemia differ from those in classical second window. Am J Physiol Heart Circ Physiol 299: H752-H762, 2010.

78. Di Lisa F and Bernardi P. A CaPful of mechanisms regulating the mitochondrial permeability transition. J Mol Cell Cardiol 46: 775-778, 2009.

79. Di Lisa F and Ziegler M. Pathophysiological relevance of mitochondria in $\mathrm{NAD}^{+}$metabolism. FEBS Lett 492: 4-8, 2001.

80. Di Lisa F, Blank PS, Colonna R, Gambassi G, Silverman HS, Stern MD, and Hansford RG. Mitochondrial membrane potential in single living adult rat cardiac myocytes exposed to anoxia or metabolic inhibition. J Physiol 486: 1-13, 1995.

81. Di Lisa F, Canton M, Carpi A, Kaludercic N, Menabò R, Menazza S, and Semenzato M. Mitochondrial injury and protection in ischemic pre- and post-conditioning. Antioxid Redox Signal 14: 881-891, 2011.

82. Di Lisa F, Kaludercic N, Carpi A, Menabò R, and Giorgio M. Mitochondrial pathways for ROS formation and myocardial injury: the relevance of p66(Shc) and monoamine oxidase. Basic Res Cardiol 104: 131-139, 2009.

83. Di Lisa F, Menabò R, Canton M, Barile M, and Bernardi P. Opening of the mitochondrial permeability transition pore causes depletion of mitochondrial and cytosolic $\mathrm{NAD}^{+}$and is a causative event in the death of myocytes in postischemic reperfusion of the heart. J Biol Chem 276: 2571-2575, 2001.

84. Dimmer KS and Scorrano L. (De)constructing mitochondria: what for? Physiology 21: 233-241, 2006.

85. Dong Y, Undyala VV, Gottlieb RA, Mentzer RM, Jr., and Przyklenk K. Autophagy: definition, molecular machinery and potential role in myocardial ischemia-reperfusion injury. J Cardiovasc Pharm Ther 15: 220-230, 2010.

86. Dong Y, Zhang M, Liang B, Xie Z, Zhao Z, Asfa S, Choi $\mathrm{HC}$, and Zou MH. Reduction of AMP-activated protein kinase $\alpha 2$ increases endoplasmic reticulum stress and atherosclerosis in vivo. Circulation 121: 792-803, 2010.

87. Dos Santos P, Laclau MN, Boudina S, and Garlid KD. Alterations of the bioenergetics systems of the cell in acute and chronic myocardial ischemia. Mol Cell Biochem 256-257: 157-166, 2004.

88. Dosenko VE, Nagibin VS, Tumanovskaya LV, Moibenko AA, and Vaage J. Postconditioning prevents apoptotic necrotic and autophagic cardiomyocyte cell death in culture. Fiziol Zh 51: 12-17, 2005.

89. Dost T, Cohen MV, and Downey JM. Redox signaling triggers protection during the reperfusion rather than the ischemic phase of preconditioning. Basic Res Cardiol 103: 378-384, 2008.

90. Downey JM and Cohen MV. We think we see a pattern emerging here. Circulation 111: 120-121, 2005.

91. Dröge W. Free radicals in the physiological control of cell function. Physiol Rev 82: 47-95, 2002.

92. Ebrahim Z, Yellon DM, and Baxter GF. Attenuated cardioprotective response to bradykinin, but not classical preconditioning, in DOCA-salt hypertensive left ventricular hypertrophy. Pharmacol Res 55: 42-48, 2007.

93. Ebrahim Z, Yellon DM, and Baxter GF. Ischemic preconditioning is lost in aging hypertensive rat heart: independent effects of aging and longstanding hypertension. Exp Gerontol 42: 807-814, 2007.

94. Echtay KS, Esteves TC, Pakay JL, Jekabsons MB, Lambert AJ, Portero-Otin M, Pamplona R, Vidal-Puig AJ, Wang S,
Roebuck SJ, and Brand MD. A signalling role for 4-hydroxy-2-nonenal in regulation of mitochondrial uncoupling. EMBO J 22: 4103-4110, 2003.

95. Echtay KS, Pakay JL, Esteves TC, and Brand MD. Hydroxynonenal and uncoupling proteins: a model for protection against oxidative damage. Biofactors 24: 119-130, 2005.

96. Echtay KS, Roussel D, St-Pierre J, Jekabsons MB, Cadenas S, Stuart JA, Harper JA, Roebuck SJ, Morrison A, Pickering S, Clapham JC, and Brand MD. Superoxide activates mitochondrial uncoupling proteins. Nature 415: 96-99, 2002.

97. Eckel RH, Grundy SM, and Zimmet PZ. The metabolic syndrome. Lancet 365: 1415-1428, 2005.

98. Eliseev RA, Malecki J, Lester T, Zhang Y, Humphrey J, and Gunter TE. Cyclophilin D interacts with Bcl2 and exerts an anti-apoptotic effect. J Biol Chem 284: 9692-9699, 2009.

99. Emerling BM, Weinberg F, Snyder C, Burgess Z, Mutlu GM, Viollet B, Budinger GR, and Chandel NS. Hypoxic activation of AMPK is dependent on mitochondrial ROS but independent of an increase in AMP/ATP ratio. Free Radic Biol Med 46: 1386-1391, 2009.

100. Endo T and Yamano K. Multiple pathways for mitochondrial protein traffic. Biol Chem 390: 723-730, 2009.

101. Esterbauer H, Schaur RJ, and Zollner H. Chemistry and biochemistry of 4-hydroxynonenal, malonaldehyde and related aldehydes. Free Radical Biol Med 11: 81-128, 1991.

102. Facundo HT, de Paula JG, and Kowaltowski AJ. Mitochondrial ATP-sensitive $\mathrm{K}^{+}$channels are redox-sensitive pathways that control reactive oxygen species production Free Radic Biol Med 42: 1039-1048, 2007.

103. Fantinelli JC and Mosca SM. Comparative effects of ischemic pre and postconditioning on ischemia-reperfusion injury in spontaneously hypertensive rats (SHR). Mol Cell Biochem 296: 45-51, 2007.

104. Ferdinandy P, Schulz R, and Baxter GF. Interaction of cardiovascular risk factors with myocardial ischemia/reperfusion injury, preconditioning, and postconditioning. Pharmacol Rev 59: 418-458, 2007.

105. Ferrara N, Rinaldi B, Corbi G, Conti V, Stiuso P, Boccuti S, Rengo G, Rossi F, and Filippelli A. Exercise training promotes SIRT1 activity in aged rats. Rejuvenation Res 11: 139150, 2008.

106. Finocchietto PV, Franco MC, Holod S, Gonzalez AS, Converso DP, Antico Arciuch VG, Serra MP, Poderoso JJ, and Carreras MC. Mitochondrial nitric oxide synthase: a masterpiece of metabolic adaptation, cell growth, transformation, and death. Exp Biol Med (Maywood) 234: 1020-1028, 2009.

107. Fischer $\mathrm{P}$ and Hilfiker-Kleiner D. Survival pathways in hypertrophy and heart failure: the gp130-STAT3 axis. Basic Res Cardiol 102: 279-297, 2007.

108. Fonseca VA. The metabolic syndrome, hyperlipidemia, and insulin resistance. Clin Cornerstone 7: 61-72, 2005.

109. Forbes RA, Steenbergen C, and Murphy E. Diazoxide induced cardioprotection requires signaling through a redoxsensitive mechanism. Circ Res 88: 802-809, 2001.

110. Fornazari M, de Paula JG, Castilho RF, and Kowaltowski AJ. Redox properties of the adenoside triphosphate-sensitive $\mathrm{K}^{+}$channel in brain mitochondria J Neurosci Res 86: 1548-1556, 2008.

111. Freixa $X$, Bellera N, Ortiz-Pérez JT, Jiménez $M$, Paré $C$, Bosch X, De Caralt TM, Betriu A, and Masotti M. Ischaemic postconditioning revisited: lack of effects on infarct size following primary percutaneous coronary intervention. Eur Heart J 33: 103-312, 2012. 
112. Fujita $M$, Asanuma $H$, Hirata $A$, Wakeno $M$, Takahama $H$, Sasaki H, Kim J, Takashima S, Tsukamoto O, Minamino T, Shinozaki Y, Tomoike H, Hori M, and Kitakaze M. Prolonged transient acidosis during early reperfusion contributes to the cardioprotective effects of postconditioning. Am J Physiol Heart Circ Physiol 292: H2004-H2008, 2007.

113. Garlid KD, Costa AD, Quinlan CL, Pierre SV, and Dos Santos P. Cardioprotective signaling to mitochondria. J Mol Cell Cardiol 46: 858-866, 2009.

114. Giorgio M, Migliaccio E, Orsini F, Paolucci D, Moroni M, Contursi C, Pelliccia G, Luzi L, Minucci S, Marcaccio M, Pinton P, Rizzuto R, Bernardi P, Paolucci F, and Pelicci PG. Electron transfer between cytochrome $\mathrm{c}$ and p66(Shc) generates reactive oxygen species that trigger mitochondrial apoptosis. Cell 122: 221-233, 2005.

115. Golstein P and Kroemer G. A multiplicity of cell death pathways. Symposium on apoptotic and non-apoptotic cell death pathways. EMBO Rep 8: 829-833, 2007.

116. Golstein P and Kroemer G. Cell death by necrosis: towards a molecular definition. Trends Biochem Sci 32: 7-43, 2007.

117. Gomez L, Paillard M, Thibault H, Derumeaux G, and Ovize M. Inhibition of GSK3 $\beta$ by postconditioning is required to prevent opening of the mitochondrial permeability transition pore during reperfusion. Circulation 117: 2761-2768, 2008.

118. Gottlieb RA and Gustafsson AB. Mitochondrial turnover in the heart. Biochim Biophys Acta 1813: 1295-1301, 2011.

119. Green DR and Kroemer G. The pathophysiology of mitochondrial cell death. Science 305: 626-629, 2004.

120. Green K, Brand MD, and Murphy MP. Prevention of mitochondrial oxidative damage as a therapeutic strategy in diabetes. Diabetes 53: S110-S118, 2004

121. Griffiths EJ and Halestrap AP. Mitochondrial non-specific pores remain closed during cardiac ischaemia, but open upon reperfusion. Biochem J 307: 93-98, 1995.

122. Griffiths EJ and Halestrap AP. Protection by Cyclosporin A of ischemia/reperfusion-induced damage in isolated rat hearts. J Mol Cell Cardiol 25: 1461-1469, 1993.

123. Gross ER, Hsu AK, and Gross GJ. Diabetes abolishes morphine-induced cardioprotection via multiple pathways upstream of glycogen synthase kinase- $3 \beta$. Diabetes 56 : $127-$ 136, 2007.

124. Grover GJ, Atwal KS, Sleph PG, Wang FL, Monshizadegan $\mathrm{H}$, Monticello T, and Green DW. Excessive ATP hydrolysis in ischemic myocardium by mitochondrial F1F0-ATPase: effect of selective pharmacological inhibition of mitochondrial ATPase hydrolase activity. Am J Physiol Heart Circ Physiol 287: H1747-H1755, 2004.

125. Guth BD, Schulz R, and Heusch G. Time course and mechanisms of contractile dysfunction during acute myocardial ischemia. Circulation 87: IV35-42, 1993.

126. Hagen $\mathrm{T}$ and Vidal-Puig A. Mitochondrial uncoupling proteins in human physiology and disease. Minerva Med 93: 41-57, 2002.

127. Halestrap AP and Pasdois P. The role of the mitochondrial permeability transition pore in heart disease. Biochim Biophys Acta 1787: 1402-1415, 2009.

128. Halestrap AP, Clarke SJ, and Javadov SA. Mitochondrial permeability transition pore opening during myocardial reperfusion-a target for cardioprotection. Cardiovasc Res 61: 372-385, 2004.

129. Hanley PJ, Mickel M, Löffler M, Brandt U, and Daut J. K(ATP) channel-independent targets of diazoxide and 5hydroxydecanoate in the heart. J Physiol 542: 735-741, 2002.
130. Hanley PJ, Ray J, Brandt U, and Daut J. Halothane, isoflurane and sevoflurane inhibit NADH: ubiquinone oxidoreductase (complex I) of cardiac mitochondria. J Physiol 544: 687-693, 2002.

131. Hardie DG. AMP-activated protein kinase as a drug target. Annu Rev Pharmacol Toxicol 47: 185-210, 2007.

132. Hausenloy DJ and Yellon DM. New directions for protecting the heart against ischaemia-reperfusion injury: targeting the Reperfusion Injury Salvage Kinase (RISK)pathway. Cardiovasc Res 61: 448-460, 2004.

133. Hausenloy DJ and Yellon DM. Preconditioning and postconditioning: united at reperfusion. Pharmacol Ther 116: 173-191, 2007.

134. Hausenloy DJ, Baxter G, Bell R, Bøtker HE, Davidson SM, Downey J, Heusch G, Kitakaze M, Lecour S, Mentzer R, Mocanu MM, Ovize M, Schulz R, Shannon R, Walker M, Walkinshaw G, and Yellon DM. Translating novel strategies for cardioprotection: the Hatter Workshop Recommendations. Basic Res Cardiol 105: 677-686, 2010.

135. Hausenloy DJ, Duchen MR, and Yellon DM. Inhibiting mitochondrial permeability transition pore opening at reperfusion protects against ischaemia-reperfusion injury. Cardiovasc Res 60: 617-625, 2003.

136. Hausenloy DJ, Lecour S, and Yellon DM. RISK and SAFE pro-survival signaling pathways in ischemic postconditioning: two sides of the same coin. Antioxid Redox Signal 14: 893-907, 2011.

137. Hausenloy DJ, Lim SY, Ong SG, Davidson SM, and Yellon DM. Mitochondrial cyclophilin-D as a critical mediator of ischaemic preconditioning. Cardiovasc Res 88: 67-74, 2010.

138. Hausenloy DJ, Ong SB, and Yellon DM. The mitochondrial permeability transition pore as a target for preconditioning and postconditioning. Basic Res Cardiol 104: 189-202, 2009.

139. Hausenloy DJ, Wynne AM, and Yellon DM. Ischemic preconditioning targets the reperfusion phase. Basic Res Cardiol 102: 445-452, 2007.

140. Hausenloy DJ, Wynne AM, Duchen MR, and Yellon D. Transient mitochondrial permeability transition pore opening mediates preconditioning-induced protection. Circulation 109: 1714-1717, 2004.

141. Hausenloy DJ. Signalling pathways in ischaemic postconditioning. Thromb Haemost 101: 626-634, 2009.

142. Heinzel FR, Luo Y, Li X, Boengler K, Buechert A, GarcíaDorado D, Di Lisa F, Schulz R, and Heusch G. Impairment of diazoxide-induced formation of reactive oxygen species and loss of cardioprotection in connexin 43 deficient mice. Circ Res 97: 583-586, 2005.

143. Heusch G and Schulz R. Preservation of peripheral vasodilation as a surrogate of cardioprotection? The mechanistic role of ATP-dependent potassium channels and the mitochondrial permeability transition pore. Eur Heart J 32: 118411862011.

144. Heusch G, Boengler K, and Schulz R. Cardioprotection: nitric oxide, protein kinases, and mitochondria. Circulation 118: 1915-1919, 2008.

145. Heusch G, Boengler K, and Schulz R. Inhibition of mitochondrial permeability transition pore opening: the holy grail of cardioprotection. Basic Res Cardiol 105: 151-154, 2010.

146. Heusch G, Büchert A, Feldhaus S, and Schulz R. No loss of cardioprotection by postconditioning in connexin 43-deficient mice. Basic Res Cardiol 101: 354-356, 2006.

147. Heusch G, Kleinbongard P, Skyschally A, Levkau B, Schulz $\mathrm{R}$, and Erbel $\mathrm{R}$. The coronary circulation in cardioprotec- 
tion: more than just one confounder. Cardiovasc Res 2011 [Epub ahead of print]; Doi: 10.1093/cvr/cvr271

148. Heusch G, Musiolik J, Kottenberg E, Peters J, Jakob H, and Thielmann M. STAT-5 activation and cardioprotection by remote ischemic preconditioning in humans: short communication. Circ Res 110: 111-115, 2012.

149. Heusch G, Skyschally A, Gres P, van Caster P, Schilawa D, and Schulz R. Improvement of regional myocardial blood flow and function and reduction of infarct size with ivabradine- protection beyond heart rate reduction. Eur Heart J 29: 2265-2275, 2008.

150. Heusch G. Nitroglycerin and delayed preconditioning in humans: yet another new mechanism for an old drug? Circulation 103: 2876-2878, 2001.

151. Heusch G. No risk, no...cardioprotection? A critical perspective. Cardiovasc Res 84: 173-175, 2009.

152. Heusch G. Obesity-a risk factor or a RISK factor for myocardial infarction? Br J Pharmacol 149: 1-3, 2006.

153. Heusch G. Postconditioning. Old wine in a new bottle? J Am Coll Cardiol 44: 1111-1112, 2004.

154. Heusch G. Reduction of infarct size by ischaemic postconditioning in humans: fact or fiction? Eur Heart J 33: 1315, 2012.

155. Hilfiker-Kleiner D, Hilfiker A, Fuchs M, Kaminski K, Schaefer A, Schieffer B, Hillmer A, Schmiedl A, Ding Z, Podewski E, Podewski E, Poli V, Schneider MD, Schulz R, Park JK, Wollert KC, and Drexler H. Signal transducer and activator of transcription 3 is required for myocardial capillary growth, control of interstitial matrix deposition, and heart protection from ischemic injury. Circ Res 95: 187-195, 2004.

156. Hilfiker-Kleiner D. Survival pathways in hypertrophy and heart failure: the gp130-STAT-3 axis. Basic Res Cardiol 102: 279-297, 2007.

157. Hoerter J, Gonzalez-Barroso MD, Couplan E, Mateo P, Gelly C, Cassard-Doulcier AM, Diolez P, and Bouillaud F. Mitochondrial uncoupling protein 1expressed in the heart of transgenic mice protects against ischemic-reperfusion damage. Circulation 110: 528-533, 2004.

158. Hoshi M, Takashima A, Noguchi K, Murayama M, Sato M, Kondo S, Saitoh Y, Ishiguro K, Hoshino T, and Imahori K. Regulation of mitochondrial pyruvate dehydrogenase activity by tau protein kinase I/glycogen synthase kinase 3beta in brain. Proc Natl Acad Sci U S A 93: 2719-2723, 1996.

159. Hoshino A, Matoba S, Iwai-Kanai E, Nakamura H, Kimata M, Nakaoka M, Katamura M, Okawa Y, Ariyoshi M, Mita Y, Ikeda K, Ueyama T, Okigaki M, and Matsubara H. p53TIGAR axis attenuates mitophagy to exacerbate cardiac damage after ischemia. J Mol Cell Cardiol 52: 175-184, 2012.

160. Hu Q, Suzuki G, Young RF, Page BJ, Fallavollita JA, and Canty JM, Jr. Reductions in mitochondrial O(2) consumption and preservation of high-energy phosphate levels after simulated ischemia in chronic hibernating myocardium. Am J Physiol Heart Circ Physiol 297: H223-H232, 2009.

161. Huang C, Andres AM, Ratliff EP, Hernandez G, Lee P, and Gottlieb RA. Preconditioning involves selective mitophagy mediated by Parkin and p62/SQSTM1. PLoS One 6: e20975, 2011.

162. Huang C, Liu W, Perry CN, Yitzhaki S, Lee Y, Yuan H, Tsukada YT, Hamacher-Brady A, Mentzer RM, Jr., and Gottlieb RA. Autophagy and protein kinase $C$ are required for cardioprotection by sulfaphenazole. Am J Physiol Heart Circ Physiol 298: H570-H579, 2010.

163. Huang C, Yitzhaki S, Perry CN, Liu W, Giricz Z, Mentzer RM, Jr., and Gottlieb RA. Autophagy induced by ischemic preconditioning is essential for cardioprotection. J Cardiovasc Transl Res 3: 365-373, 2010.

164. Huhn R, Heinen A, Hollmann MW, Schlack W, Preckel B, and Weber NC. Cyclosporine A administered during reperfusion fails to restore cardioprotection in prediabetic Zucker obese rats in vivo. Nutr Metab Cardiovasc Dis 20: 706712, 2010.

165. Huhn R, Heinen A, Weber NC, Hollmann MW, Schlack W, and Preckel B. Hyperglycaemia blocks sevoflurane-induced postconditioning in the rat heart in vivo: cardioprotection can be restored by blocking the mitochondrial permeability transition pore. Br J Anaesth 100: 465-471, 2008.

166. Huisamen B. Protein kinase B in the diabetic heart. Mol Cell Biochem 249: 31-38, 2003.

167. Hunter DR, Haworth RA, and Southard JH. Relationship between configuration, function, and permeability in calciumtreated mitochondria. J Biol Chem 251: 5069-5077, 1976.

168. Hunter JC and Korzick DH. Age- and sex-dependent alterations in protein kinase $\mathrm{C}$ (PKC) and extracellular regulated kinase 1/2 (ERK1/2) in rat myocardium. Mech Ageing Dev 126: 535-550, 2005.

169. Husainy MA, Dickenson JM, and Galiñanes M. The MPTP status during early reoxygenation is critical for cardioprotection. J Surg Res 174: 62-72, 2012.

170. Hüser J and Blatter LA. Fluctuations in mitochondrial membrane potential caused by repetitive gating of the permeability transition pore. Biochem J 343: 311-317, 1999.

171. Ichas F and Mazat JP. From calcium signaling to cell death: two conformations for the mitochondrial permeability transition pore. Switching from low- to high-conductance state. Biochim Biophys Acta 1366: 33-50, 1998.

172. Ichas F, Jouaville LS, and Mazat JP. Mitochondria are excitable organelles capable of generating and conveying electrical and calcium signals. Cell 89: 1145-1153, 1997.

173. Iliodromitis EK, Andreadou I, Prokovas E, Zoga A, Farmakis D, Fotopoulou T, Ioannidis K, Paraskevaidis IA, and Kremastinos DT. Simvastatin in contrast to postconditioning reduces infarct size in hyperlipidemic rabbits: possible role of oxidative/nitrosative stress attenuation. Basic Res Cardiol 105: 193-203, 2010.

174. Iliodromitis EK, Downey JM, Heusch G, and Kremastinos DT. What is the optimal postconditioning algorithm? J Cardiovasc Pharmacol Ther 14: 269-273, 2009.

175. Inserte J, Ruiz-Meana M, Rodriguez-Sinovas A, Barba I, and Garcia-Dorado D. Contribution of delayed intracellular $\mathrm{pH}$ recovery to ischemic postconditioning protection. Antioxid Redox Signal 14: 923-939, 2011.

176. Irrcher I, Ljubicic V, and Hood DA. Interactions between ROS and AMP kinase activity in the regulation of PGC1 $\alpha$ transcription in skeletal muscle cells. Am J Physiol Cell Physiol 296: C116-C123, 2009.

177. Irrcher I, Ljubicic V, Kirwan AF, and Hood DA. AMP-activated protein kinase-regulated activation of the PGC1 $\alpha$ promoter in skeletal muscle cells. PLoS One 3: e3614, 2008.

178. Ishii H, Ichimiya S, Kanashiro M, Amano T, Imai K, Murohara $\mathrm{T}$, and Matsubara T. Impact of a single intravenous administration of nicorandil before reperfusion in patients with ST-segment-elevation myocardial infarction. Circulation 112: 1284-1288, 2005.

179. Ivanes F, Rioufol G, Piot C, and Ovize M. Postconditioning in acute myocardial infarction patients. Antioxid Redox Signal 14: 811-820, 2011.

180. Jacobson J and Duchen MR. Mitochondrial oxidative stress and cell death in astrocytes-requirement for stored $\mathrm{Ca}^{2+}$ 
and sustained opening of the permeability transition pore. $J$ Cell Sci 115: 1175-1188, 2002.

181. Jacoby JJ, Kalinowski A, Liu MG, Zhang SS, Gao Q, Chai GX, Ji L, Iwamoto Y, Li E, Schneider M, Russell KS, and Fu XY. Cardiomyocyte-restricted knockout of STAT3 results in higher sensitivity to inflammation, cardiac fibrosis, and heart failure with advanced age. Proc Natl Acad Sci U S A 100: 12929-12934, 2003.

182. Jalowy A, Schulz R, Post H, Dörge H, Weidenbach R, and Heusch G. The angiotensin II AT1-receptor antagonist candesartan reduces infarct size in pigs. FASEB J 12: A1114, 1998.

183. Jaswal JS, Lund CR, Keung W, Beker DL, Rebeyka IM, and Lopaschuk GD. Isoproterenol stimulates 5'-AMP-activated protein kinase and fatty acid oxidation in neonatal hearts. Am J Physiol Heart Circ Physiol 299: H1135-H1145, 2010.

184. Javadov SA, Clarke S, Das M, Griffiths EJ, Lim KH, and Halestrap AP. Ischaemic preconditioning inhibits opening of mitochondrial permeability transition pores in the reperfused rat heart. J Physiol 549: 513-524, 2003.

185. Juhaszova M, Zorov DB, Kim SH, Pepe S, Fu Q, Fishbein $\mathrm{KW}$, Ziman BD, Wang S, Ytrehus K, Antos CL, Olson EN, and Sollott SJ. Glycogen synthase kinase- $3 \beta$ mediates convergence of protection signaling to inhibit the mitochondrial permeability transition pore. J Clin Invest 113: 15351549, 2004.

186. Juhaszova M, Zorov DB, Yaniv $Y$, Nuss HB, Wang S, and Sollott SJ. Role of glycogen synthase kinase- $3 \beta$ in cardioprotection. Circ Res 104: 1240-1252, 2009.

187. Kageyama H, Suga A, Kashiba M, Oka J, Osaka T, Kashiwa T, Hirano T, Nemoto K, Namba Y, Ricquier D, Giacobino $\mathrm{JP}$, and Inoue $\mathrm{S}$. Increased uncoupling protein-2 and -3 gene expressions in skeletal muscle of STZ-induced diabetic rats. FEBS Lett 440: 450-453, 1998.

188. Kanai AJ, Pearce LL, Clemens PR, Birder LA, VanBibber MM, Choi SY, de Groat WC, and Peterson J. Identification of a neuronal nitric oxide synthase in isolated cardiac mitochondria using electrochemical detection. Proc Natl Acad Sci U S A 98: 14126-14131, 2001.

189. Kelly DE, He J, Menshikova EV, and Ritov VB. Dysfunction of mitochondria in human skeletal muscle in type 2 diabetes. Diabetes 51: 2944-2950, 2002.

190. Kersten JR, Toller WG, Gross ER, Pagel PS, and Warltier DC. Diabetes abolishes ischemic preconditioning: role of glucose, insulin, and osmolality. Am J Physiol Heart Circ Physiol 278: H1218-H1224, 2000.

191. Kevin LG, Camara AK, Riess ML, Novalija E, and Stowe DF. Ischemic preconditioning alters real-time measure of $\mathrm{O} 2$ radicals in intact hearts with ischemia and reperfusion. Am J Physiol Heart Circ Physiol 284: H566-H574, 2003.

192. Kin H, Wang NP, Mykytenko J, Reeves J, Deneve J, Jiang R, Zatta AJ, Guyton RA, Vinten-Johansen J, and Zhao ZQ. Inhibition of myocardial apoptosis by postconditioning is associated with attenuation of oxidative stress-mediated nuclear factor-kappa B translocation and TNF alpha release. Shock 29: 761-768, 2008.

193. Kin H, Zhao ZQ, Sun HY, Wang NP, Corvera JS, Halkos ME, Kerendi F, Guyton RA, and Vinten-Johansen J. Postconditioning attenuates myocardial ischemia-reperfusion injury by inhibiting events in the early minutes of reperfusion. Cardiovasc Res 62: 74-85, 2004.

194. Kinnally KW and Antonsson B. A tale of two mitochondrial channels, MAC and PTP, in apoptosis. Apoptosis 12: 857868, 2007.
195. Kitakaze M, Asakura M, Kim J, Shintani Y, Asanuma H, Hamasaki T, Seguchi O, Myoishi M, Minamino T, Ohara T, Nagai Y, Nanto S, Watanabe K, Fukuzawa S, Hirayama A, Nakamura N, Kimura K, Fujii K, Ishihara M, Saito Y, Tomoike H, Kitamura S, and J-WIND investigators. Human atrial natriuretic peptide and nicorandil as adjuncts to reperfusion treatment for acute myocardial infarction ( JWIND): two randomised trials. Lancet 370: 1483-1493, 2007.

196. Klionsky DJ, Abeliovich H, Agostinis P, Agrawal DK, Aliev $\mathrm{G}$, Askew DS, et al. Guidelines for the use and interpretation of assays for monitoring autophagy in higher eukaryotes Autophagy 4: 151-175, 2008.

197. Kokoszka JE, Waymire KG, Levy SE, Sligh JE, Cai J, Jones DP, MacGregor GR, and Wallace DC. The ADP/ATP translocator is not essential for the mitochondrial permeability transition pore. Nature 427: 461-465, 2004.

198. Korge P, Yang L, Yang JH, Wang Y, Qu Z, and Weiss JN. Protective role of transient pore openings in calcium handling by cardiac mitochondria. J Biol Chem 286: 348-357, 2011.

199. Korichneva I. Zinc dynamics in the myocardial redox signaling network. Antioxid Redox Signal 8: 1707-1721, 2006.

200. Korzick DH, Holiman DA, Boluyt MO, Laughlin MH, and Lakatta EG. Diminished alpha1-adrenergic-mediated contraction and translocation of PKC in senescent rat heart. Am J Physiol Heart Circ Physiol 281: H581-H589, 2001.

201. Korzick DH, Laughlin MH, and Bowles DK. Alterations in PKC signaling underlie enhanced myogenic tone in exercise-trained porcine coronary resistance arteries. J Appl Physiol 96: 1425-1432, 2004.

202. Kristian T, Bernardi P, and Siesjo BK. Acidosis promotes the permeability transition in energized mitochondria: implications for reperfusion injury. J Neurotrauma 18: 10591074, 2001.

203. Kristiansen SB, Løfgren B, Støttrup NB, Khatir D, NielsenKudsk JE, Nielsen TT, Bøtker HE, and Flyvbjerg A. Ischaemic preconditioning does not protect the heart in obese and lean animal models of type 2 diabetes. Diabetologia 47: 1716-1721, 2004.

204. Kukidome D, Nishikawa T, Sonoda K, Imoto K, Fujisawa K, Yano M, Motoshima H, Taguchi T, Matsumura T, and Araki E. Activation of AMP-activated protein kinase reduces hyperglycemia-induced mitochondrial reactive oxygen species production and promotes mitochondrial biogenesis in human umbilical vein endothelial cells. Diabetes 55: 120-127, 2006.

205. Kupai K, Csonka C, Fekete V, Odendaal L, van Rooyen J, Marais de W, Csont T, and Ferdinandy P. Cholesterol dietinduced hyperlipidemia impairs the cardioprotective effect of postconditioning: role of peroxynitrite. Am J Physiol Heart Circ Physiol 297: H1729-H1735, 2009.

206. Kupatt C, Hinkel R, Horstkotte J, Deiß M, von Brühl M-L, Bilzer M, and Boekstegers P. Selective retroinfusion of GSH and cariporide attenuates myocardial ischemia-reperfusion injury in a preclinical pig model. Cardiovasc Res 61: 530-537, 2004.

207. Kutala VK, Khan M, Angelos MG, and Kuppusamy P. Role of oxygen in postischemic myocardial injury. Antioxid Redox Signal 9: 1193-1206, 2007.

208. Ladilov Y, Efe O, Schäfer C, Rother B, Kasseckert S, Abdallah Y, Meuter K, Dieter-Schlüter K, and Piper HM. Reoxygenation-induced rigor-type contracture. J Mol Cell Cardiol 35: 1481-1490, 2003.

209. Laky D and Parascan L. Hibernating myocardium, morphological studies on intraoperatory myocardial biopsies 
and on chronic ischemia experimental model. Rom J Morphol Embryol 48: 407-413, 2007.

210. Lal H, Zhou J, Ahmad F, Zaka R, Vagnozzi RJ, Decaul M, Woodgett J, Gao E, and Force T. Glycogen synthase kinase$3 \alpha$ limits ischemic injury, cardiac rupture, post-myocardial infarction remodeling and death. Circulation 125: 65-75, 2012.

211. Lambeth JD. Nox enzymes and the biology of reactive oxygen. Nat Rev Immunol 4: 181-189, 2004.

212. Lecour S. Activation of the protective Survivor Activating Factor Enhancement (SAFE) pathway against reperfusion injury: does it go beyond the RISK pathway? J Mol Cell Cardiol 47: 32-40, 2009.

213. Lemasters JJ. Selective mitochondrial autophagy, or mitophagy, as a targeted defense against oxidative stress, mitochondrial dysfunction, and aging. Rejuvenation Res 8: 3-5, 2005.

214. Lemoine S, Buléon C, Rouet R, Ivascau C, Babatasi G, Massetti M, Gérard JL, and Hanouz JL. Bradykinin and adenosine receptors mediate desflurane induced postconditioning in human myocardium: role of reactive oxygen species. BMC Anesthesiol 10: 12, 2010.

215. Li XM, Ma YT, Yang YN, Zhang JF, Chen BD, Liu F, Huang $\mathrm{Y}$, Han W, and Gao XM. Ischemic postconditioning protects hypertrophic myocardium by ERK1/2 signaling pathway: experiment with mice. Zhonghua Yi Xue Za Zhi 89: 846-850, 2009.

216. Light PE. Cardiac $\mathrm{K}_{\mathrm{ATP}}$ channels and ischemic preconditioning: current perspectives. Can J Cardiol 15: 1123-1130, 1999.

217. Lilly M, Sandholm J, Cooper JJ, Koskinen PJ, and Kraft A. The PIM-1 serine kinase prolongs survival and inhibits apoptosis-related mitochondrial dysfunction in part through a bcl-2-dependent pathway. Oncogene 18: 4022-4031, 1999.

218. Lin J, Handschin C, and Spiegelman BM. Metabolic control through the PGC-1 family of transcription coactivators. Cell Metab 1: 361-370, 2005.

219. Linnane AW and Eastwood H. Cellular redox regulation and prooxidant signaling systems: a new perspective on the free radical theory of aging. Ann NY Acad Sci 1067: 47$55,2006$.

220. Liu B, Tewari AK, Zhang L, Green-Church KB, Zweier JL, Chen YR, and He G. Proteomic analysis of protein tyrosine nitration after ischemia reperfusion injury: mitochondria as the major target. Biochim Biophys Acta 1794: 476-485, 2009.

221. Lloyd-Jones D, Adams RJ, Brown TM, Carnethon M, Dai S, De Simone G, Ferguson TB, Ford E, Furie K, Gillespie C, Go A, Greenlund K, Haase N, Hailpern S, Ho PM, Howard V, Kissela B, Kittner S, Lackland D, Lisabeth L, Marelli A, McDermott MM, Meigs J, Mozaffarian D, Mussolino M, Nichol G, Roger VL, Rosamond W, Sacco R, Sorlie P, Stafford R, Thom T, Wasserthiel-Smoller S, Wong ND, and Wylie-Rosett J. Heart disease and stroke statistics-2010 update: a report from the american heart association. Circulation 121: e46-e215, 2010.

222. Loos B, Genade S, Ellis B, Lochner A, and Engelbrecht AM. At the core of survival: autophagy delays the onset of both apoptotic and necrotic cell death in a model of ischemic cell injury. Exp Cell Res 317: 1437-1453, 2011.

223. Luo W, Li B, Chen R, Huang R, and Lin G. Effect of ischemic postconditioning in adult valve replacement. Eur J Cardiothorac Surg 33: 203-208, 2008.

224. Ma H, Guo R, Yu L, Zhang Y, and Ren J. Aldehyde dehydrogenase 2 (ALDH2) rescues myocardial ischaemia/ reperfusion injury: role of autophagy paradox and toxic aldehyde. Eur Heart J 32: 1025-1038, 2011.
225. Ma X, Zhang X, Li C, and Luo M. Effect of postconditioning on coronary blood flow velocity and endothelial function and LV recovery after myocardial infarction. J Interv Cardiol 19: 367-375, 2006.

226. Manintveld OC, Te Lintel Hekkert M, van den Bos EJ, Suurenbroek GM, Dekkers DH, Verdouw PD, Lamers JM, and Duncker DJ. Cardiac effects of postconditioning depend critically on the duration of index ischemia. Am J Physiol Heart Circ Physiol 292: H1551-H1560, 2007.

227. McCully JD, Uematsu M, Parker RA, and Levitsky S. Adenosine-enhanced ischemic preconditioning provides enhanced cardioprotection in the aged heart. Ann Thorac Surg 66: 2037-2043, 1998.

228. McFalls EO, Sluiter W, Schoonderwoerd K, Manintveld OC, Lamers JM, Bezstarosti K, van Beusekom HM, Sikora J, Ward HB, Merkus D, and Duncker DJ. Mitochondrial adaptations within chronically ischemic swine myocardium. J Mol Cell Cardiol 41: 980-988, 2006.

229. McLeod CJ, Aziz A, Hoyt RF, Jr., McCoy JP, Jr., and Sack MN. Uncoupling proteins 2 and 3 function in concert to augment tolerance to cardiac ischemia. J Biol Chem 280: 33470-33476, 2005.

230. Mewton N, Croisille P, Gahide G, Rioufol G, Bonnefoy E, Sanchez I, Cung TT, Sportouch C, Angoulvant D, Finet G, André-Fouët X, Derumeaux G, Piot C, Vernhet H, Revel D, and Ovize M. Effect of cyclosporine on left ventricular remodeling after reperfused myocardial infarction. J Am Coll Cardiol 55: 1200-1205, 2010.

231. Miki T, Miura T, Hotta H, Tanno M, Yano T, Sato T, Terashima Y, Takada A, Ishikawa S, and Shimamoto K. Endoplasmic reticulum stress in diabetic hearts abolishes erythropoietin-induced myocardial protection by impairment of phospho-glycogen synthase kinase-3 beta-mediated suppression of mitochondrial permeability transition. Diabetes 58: 2863-2872, 2009.

232. Miro-Casas E, Ruiz-Meana M, Agullo E, Stahlhofen S, Rodriguez-Sinovas A, Cabestrero A, Jorge I, Torre I, Vazquez J, Boengler K, Schulz R, Heusch G, and Garcia-Dorado D. Connexin 43 in cardiomyocyte mitochondria contributes to mitochondrial potassium uptake. Cardiovasc Res 83: 747756, 2009.

233. Mudd JO and Kass DA. Tackling heart failure in the twenty-first century. Nature 451: 919-928, 2008.

234. Muraski JA, Rota M, Misao Y, Fransioli J, Cottage C, Gude N, Esposito G, Delucchi F, Arcarese M, Alvarez R, Siddiqi S, Emmanuel GN, Wu W, Fischer K, Martindale JJ, Glembotski CC, Leri A, Kajstura J, Magnuson N, Berns A, Beretta RM, Houser SR, Schaefer EM, Anversa P, and Sussman MA. Pim-1 regulates cardiomyocyte survival downstream of Akt. Nat Med 13: 1467-1475, 2007.

235. Murphy E and Steenbergen C. Gender-based differences in mechanisms of protection in myocardial ischemiareperfusion injury. Cardiovasc Res 75: 478-486, 2007.

236. Murphy E and Steenbergen C. Mechanisms underlying acute protection from cardiac ischemia-reperfusion injury. Physiol Rev 88: 581-609, 2008.

237. Murphy E, Glasgow W, Fralix T, and Steenbergen C. Role of lipoxygenase metabolites in ischemic preconditioning. Circ Res 76: 457-467, 1995.

238. Murphy MP. How mitochondria produce reactive oxygen species. Biochem J 417: 1-13, 2009.

239. Murry CE, Jennings RB, and Reimer KA. Preconditioning with ischemia: a delay of lethal cell injury in ischemic myocardium. Circulation 74: 1124-1136, 1986. 
240. Nadtochiy SM, Baker PR, Freeman BA, and Brookes PS. Mitochondrial nitroalkene formation and mild uncoupling in ischaemic preconditioning: implications for cardioprotection. Cardiovasc Res 82: 333-340, 2009.

241. Nadtochiy SM, Burwell LS, Ingraham CA, Spencer CM, Friedman E, Pinkert CA, and Brookes PS. In vivo cardioprotection by S-nitroso-2-mercaptopropionyl glycine. J Mol Cell Cardiol 46: 960-968, 2009.

242. Naito Z, Takashi E, Xu G, Ishiwata T, Teduka K, Yokoyama M, Yamada N, Sugisaki Y, and Asano G. Different influences of hyperglycemic duration on phosphorylated extracellular signal-regulated kinase 1/2 in rat heart. Exp Mol Pathol 74: 23-32, 2003.

243. Nakagawa T, Shimizu S, Watanabe T, Yamaguchi O, Otsu $\mathrm{K}$, Yamagata $\mathrm{H}$, Inohara $\mathrm{H}, \mathrm{Kubo} \mathrm{T}$, and Tsujimoto $\mathrm{Y}$. Cyclophilin D-dependent mitochondrial permeability transition regulates some necrotic but not apoptotic cell death. Nature 434: 652-658, 2005.

244. Naslund U, Haggmark S, Johansson G, Pennert K, Reiz S, and Marklund SL. Effects of reperfusion and superoxide dismutase on myocardial infarct size in a closed chest pig model. Cardiovasc Res 26: 170-178, 1992.

245. Nikolaidis LA, Mankad S, Sokos GG, Miske G, Shah A, Elahi D, and Shannon RP. Effects of glucagon-like peptide1 in patients with acute myocardial infarction and left ventricular dysfunction after successful reperfusion. Circulation 109: 962-965, 2004.

246. Nishihara M, Miura T, Miki T, Sakamoto J, Tanno M, Kobayashi H, Ikeda Y, Ohori K, Takahashi A, and Shimamoto K. Erythropoietin affords additional cardioprotection to preconditioned hearts by enhanced phosphorylation of glycogen synthase kinase-3 $\beta$. Am J Physiol Heart Circ Physiol 291: H748-H755, 2006.

247. Nishihara M, Miura T, Miki T, Tanno M, Yano T, Naitoh K, Ohori K, Hotta H, Terashima Y, and Shimamoto K. Modulation of the mitochondrial permeability transition pore complex in GSK-3beta-mediated myocardial protection. J Mol Cell Cardiol 43: 564-557, 2007.

248. Nishino Y, Webb IG, Davidson SM, Ahmed AI, Clark JE, Jacquet S, Shah AM, Miura T, Yellon DM, Avkiran M, and Marber MS. Glycogen synthase kinase-3 inactivation is not required for ischemic preconditioning or postconditioning in the mouse. Circ Res 103: 307-314, 2008.

249. Obame FN, Plin-Mercier C, Assaly R, Zini R, Dubois-Rande JL, Berdeaux A, and Morin D. Cardioprotective effect of morphine and a blocker of glycogen synthase kinase $3 \beta$, SB216763 [3-(2,4-dichlorophenyl)-4(1-methyl-1H-indol-3-yl)1Hpyrrole-2,5-dione], via inhibition of the mitochondrial permeability transition pore. J Pharmacol Exp Ther 326: 252258, 2008.

250. Obata K, Yasue H, Okumura K, Matsuyama K, Ogawa H, Kurose M, Saito Y, Nakao K, Imura H, and Nobuyoshi M. Atrial natriuretic polypeptide is removed by the lungs and released into the left atrium as well as the right atrium in humans. J Am Coll Cardiol 15: 1537-1543, 1990.

251. Ohnuma Y, Miura T, Miki T, Tanno M, Kuno A, Tsuchida $A$, and Shimamoto $K$. Opening of mitochondrial $K_{\mathrm{ATP}}$ channel occurs downstream of $\mathrm{PKC} \varepsilon$ activation in the mechanism of preconditioning. Am J Physiol Heart Circ Physiol 283: H440-H447, 2002.

252. Okado-Matsumoto A and Fridovich I. Putative denitrosylase activity of $\mathrm{Cu}, \mathrm{Zn}$-superoxide dismutase. Free Radic Biol Med 43: 830-836, 2007.
253. Oldenburg O, Qin Q, Krieg T, Yang XM, Philipp S, Critz $\mathrm{SD}$, Cohen MV, and Downey JM. Bradykinin induces mitochondrial ROS generation via NO, cGMP, PKG, and mitoK $_{\text {ATP }}$ channel opening and leads to cardioprotection. Am J Physiol Heart Circ Physiol 286: H468-H476, 2004.

254. Ong SB and Hausenloy DJ. Mitochondrial morphology and cardiovascular disease. Cardiovasc Res 88: 16-29, 2010.

255. Ong SB, Subrayan S, Lim SY, Yellon DM, Davidson SM, and Hausenloy DJ, Inhibiting mitochondrial fission protects the heart against ischemia/reperfusion injury. Circulation 121: 2012-2022, 2010.

256. Orsini F, Migliaccio E, Moroni M, Contursi C, Raker VA, Piccini D, Martin-Padura I, Pelliccia G, Trinei M, Bono M, Puri C, Tacchetti C, Ferrini M, Mannucci R, Nicoletti I, Lanfrancone L, Giorgio M, and Pelicci PG. The life span determinant p66Shc localizes to mitochondria where it associates with mitochondrial heat shock protein 70 and regulates trans-membrane potential. J Biol Chem 279: 2568925695, 2004.

257. Osbakken $\mathrm{M}$, Ito $\mathrm{K}$, Zhang $\mathrm{D}$, Ponomarenko I, Ivanics $\mathrm{T}$, Jahngen EG, and Cohn M. Creatine and cyclocreatine effects on ischemic myocardium: 31P nuclear magnetic resonance evaluation of intact heart. Cardiology 80: 184-195, 1992.

258. O'Shea JJ, Gadina M, and Schreiber RD. Cytokine signaling in 2002: new surprises in the Jak/Stat pathway. Cell 109: S121-S131, 2002.

259. Ovize M, Baxter GF, Di Lisa F, Ferdinandy P, Garcia-Dorado D, Hausenloy DJ, Heusch G, Vinten-Johansen J, Yellon DM, Schulz R; Working Group of Cellular Biology of Heart of European Society of Cardiology. Postconditioning and protection from reperfusion injury: where do we stand? Position paper from the Working Group of Cellular Biology of the Heart of the European Society of Cardiology. Cardiovasc Res 87: 406-423, 2010.

260. Pagliaro P, Moro F, Tullio F, Perrelli MG, and Penna C. Cardioprotective pathways during reperfusion: focus on redox signaling and other modalities of cell signaling. Antioxid Redox Signal 14: 833-850, 2011.

261. Paillard M, Gomez L, Augeul L, Loufouat J, Lesnefsky EJ, and Ovize M. Postconditioning inhibits MPTP opening independent of oxidative phosphorylation and membrane potential. J Mol Cell Cardiol 46: 902-909, 2009.

262. Pain T, Yang X-M, Critz SD, Yue Y, Nakano A, Liu GS, Heusch G, Cohen MV, and Downey JM. Opening of mitochondrial $\mathrm{K}_{\mathrm{ATP}}$ channels triggers the preconditioned state by generating free radicals. Circ Res 87: 460-466, 2000.

263. Paiva MA, Rutter-Locher Z, Gonçalves LM, Providência LA, Davidson SM, Yellon DM, and Mocanu MM. Enhancing AMPK activation during ischemia protects the diabetic heart against reperfusion injury. Am J Physiol Heart Circ Physiol 300: H2123-H2134, 2011.

264. Paoletti F, Aldinucci D, Mocali A, and Caparrini A. A sensitive spectrophotometric method for the determination of superoxide dismutase activity in tissue extracts. Analytical Biochemistry 154: 536-541, 1986.

265. Patel HH, Hsu AK, and Gross GJ. COX-2 and iNOS in opioid-induced delayed cardioprotection in the intact rat. Life Sci 75: 129-140, 2004.

266. Peart JN and Headrick JP. Sustained cardioprotection: exploring unconventional modalities. Vascul Pharmacol 49: 63-70, 2008.

267. Penna C, Abbadessa G, Mancardi D, Tullio F, Piccione F, Spaccamiglio A, Racca S, and Pagliaro P. Synergistic effects 
against post-ischemic cardiac dysfunction by sub-chronic nandrolone pretreatment and postconditioning: role of beta2-adrenoceptor. J Physiol Pharmacol 59: 645-659, 2008.

268. Penna C, Cappello S, Mancardi D, Raimondo S, Rastaldo R, Gattullo D, Losano G, and Pagliaro P. Post-conditioning reduces infarct size in the isolated rat heart: role of coronary flow and pressure and the nitric oxide/cGMP pathway. Basic Res Cardiol 101: 168-179, 2006.

269. Penna C, Mancardi D, Raimondo S, Geuna S, and Pagliaro $\mathrm{P}$. The paradigm of postconditioning to protect the heart. $J$ Cell Mol Med 12: 435-458, 2008.

270. Penna C, Mancardi D, Rastaldo R, and Pagliaro P. Cardioprotection: a radical view Free radicals in pre and postconditioning. Biochim Biophys Acta-Bioenerg 1787: 781-793, 2009.

271. Penna C, Mancardi D, Rastaldo R, Losano G, and Pagliaro $P$. Intermittent activation of bradykinin $B_{2}$ receptors and mitochondrial $\mathrm{K}_{\mathrm{ATP}}$ channels trigger cardiac postconditioning through redox signaling. Cardiovasc Res 75: 168-177, 2007.

272. Penna C, Mancardi D, Tullio F, and Pagliaro P. Intermittent adenosine at the beginning of reperfusion does not trigger cardioprotection. J Surg Res 153: 231-238, 2009.

273. Penna C, Mancardi D, Tullio F, and Pagliaro P. Postconditioning and intermittent bradykinin induced cardioprotection require cyclooxygenase activation and prostacyclin release during reperfusion. Basic Res Cardiol 103: 368-377, 2008.

274. Penna C, Perrelli MG, Raimondo S, Tullio F, Merlino A, Moro F, Geuna S, Mancardi D, and Pagliaro P. Postconditioning induces an anti-apoptotic effect and preserves mitochondrial integrity in isolated rat hearts. Biochim Biophys Acta-Bioenerg 1787: 794-801, 2009.

275. Penna C, Perrelli MG, Tullio F, Moro F, Parisella ML, Merlino A, and Pagliaro P. Post-ischemic early acidosis in cardiac postconditioning modifies the activity of antioxidant enzymes, reduces nitration, and favors protein Snitrosylation. Pflugers Arch 462: 219-233, 2011.

276. Penna C, Rastaldo R, Mancardi D, Raimondo S, Cappello S, Gattullo D, Losano G, and Pagliaro P. Post-conditioning induced cardioprotection requires signaling through a redox-sensitive mechanism, mitochondrial ATP-sensitive $\mathrm{K}^{+}$ channel and protein kinase $\mathrm{C}$ activation. Basic Res Cardiol 101: 180-189, 2006.

277. Penna C, Tullio F, Merlino A, Moro F, Raimondo S, Rastaldo R, Perrelli MG, Mancardi D, and Pagliaro P. Postconditioning cardioprotection against infarct size and postischemic systolic dysfunction is influenced by gender. Basic Res Cardiol 104: 390-402, 2009.

278. Penna C, Tullio F, Moro F, Folino A, Merlino A, and Pagliaro P. Effects of a protocol of ischemic postconditioning and/or captopril in hearts of normotensive and hypertensive rats. Basic Res Cardiol 105: 181-192, 2010.

279. Penna C, Tullio F, Perrelli MG, Moro F, Abbadessa G, Piccione F, Carriero V, Racca S, and Pagliaro P. Ischemia/ reperfusion injury is increased and cardioprotection by a postconditioning protocol is lost as cardiac hypertrophy develops in nandrolone treated rats. Basic Res Cardiol 106: 409-420, 2011.

280. Perseghin G, Petersen K, and Shulman GI. Cellular mechanism of insulin resistance: potential links with inflammation. Int J Obes Relat Metab Disord 27: S6-S11, 2003.

281. Peterson LR, Herrero P, Schechtman KB, Racette SB, Waggoner AD, Kisrieva-Ware Z, Dence C, Klein S, Marsala
J, Meyer T, and Gropler RJ. Effect of obesity and insulin resistance on myocardial substrate metabolism and efficiency in young women. Circulation 109: 2191-2196, 2004.

282. Petronilli V, Miotto G, Canton M, Brini M, Colonna R, Bernardi $\mathrm{P}$, and Di Lisa F. Transient and long-lasting openings of the mitochondrial permeability transition pore can be monitored directly in intact cells by changes in mitochondrial calcein fluorescence. Biophys J 76: 725-734, 1999.

283. Petronilli V, Penzo D, Scorrano L, Bernardi P, and Di Lisa F. The mitochondrial permeability transition, release of cytochrome $\mathrm{c}$ and cell death. Correlation with the duration of pore openings in situ. J Biol Chem 276: 12030-12034, 2001.

284. Piot C, Croisille P, Staat $P$, Thibault H, Rioufol G, Mewton N, Elbelghiti R, Cung TT, Bonnefoy E, Angoulvant D, Macia C, Raczka F, Sportouch C, Gahide G, Finet G, AndréFouët X, Revel D, Kirkorian G, Monassier JP, Derumeaux $\mathrm{G}$, and Ovize M. Effect of cyclosporine on reperfusion injury in acute myocardial infarction. N Engl J Med 359: 473481, 2008.

285. Piper HM, Abdallah Y, and Schäfer C. The first minutes of reperfusion: a window of opportunity for cardioprotection. Cardiovasc Res 61: 365-371, 2004.

286. Piper HM, Garcia-Dorado D, and Ovize M. A fresh look at reperfusion injury. Cardiovasc Res 38: 291-300, 1998.

287. Piper HM, Kasseckert S, and Abdallah Y. The sarcoplasmic reticulum as the primary target of reperfusion protection. Cardiovasc Res 70: 170-173, 2006.

288. Prime TA, Blaikie FH, Evans C, Nadtochiy SM, James AM, Dahm CC, Vitturi DA, Patel RP, Hiley CR, Abakumova I, Requejo R, Chouchani ET, Hurd TR, Garvey JF, Taylor CT, Brookes PS, Smith RA, and Murphy MP. A mitochondriatargeted S-nitrosothiol modulates respiration, nitrosates thiols, and protects against ischemia-reperfusion injury. Proc Natl Acad Sci U S A 106: 10764-10769, 2009.

289. Przyklenk K and Kloner RA. "Reperfusion injury" by oxygen-derived free radicals? Effect of superoxide dismutase plus catalase, given at the time of reperfusion, on myocardial infarct size, contractile function, coronary microvasculature, and regional myocardial blood flow. Circ Res 64: 86-96, 1989.

290. Przyklenk K, Dong Y, Undyala VV, and Whittaker P. Autophagy as a therapeutic target for ischemia-reperfusion injury? Concepts, controversies and challenges. Cardiovasc Res 2012 [Epub ahead of print]; Doi: 10.1093/cvr/cvr358

291. Przyklenk K, Li G, Simkhovich BZ, and Kloner RA. Mechanisms of myocardial ischemic preconditioning are age related: PKC-epsilon does not play a requisite role in old rabbits. J Appl Physiol 95: 2563-2569, 2003.

292. Przyklenk K, Maynard M, Darling CE, and Whittaker P. Aging mouse hearts are refractory to infarct size reduction with post-conditioning. J Am Coll Cardiol 51: 1393-1398, 2008.

293. Przyklenk K, Maynard M, Greiner DL, and Whittaker P. Cardioprotection with postconditioning: loss of efficacy in murine models of type-2 and type-1 diabetes. Antioxid Redox Signal 14: 781-790, 2011.

294. Qi D, Hu X, Wu X, Merk M, Leng L, Bucala R, and Young LH. Cardiac macrophage migration inhibitory factor inhibits JNK pathway activation and injury during ischemia/ reperfusion. J Clin Invest 119: 3807-3816, 2009.

295. Queliconi BB, Wojtovich AP, Nadtochiy SM, Kowaltowski $\mathrm{AJ}$, and Brookes PS. Redox regulation of the mitochondrial $\mathrm{K}_{\text {ATP }}$ channel in cardioprotection. Biochim Biophys Acta 1813: 1309-1315, 2011. 
296. Quinlan CL, Costa AD, Costa CL, Pierre SV, Dos Santos P, and Garlid KD. Conditioning the heart induces formation of signalosomes that interact with mitochondria to open mitoK $_{\text {ATP }}$ channels. Am J Physiol Heart Circ Physiol 295: H953-H961, 2008.

297. Rajesh KG, Sasaguri S, Zhitian Z, Suzuki R, Asakai R, and Maeda $\mathrm{H}$. Second window of ischemic preconditioning regulates mitochondrial permeability transition pore by enhancing Bcl-2 expression. Cardiovasc Res 59: 297-307, 2003.

298. Raphael J, Gozal Y, Navot N, and Zuo Z. Hyperglycemia inhibits anesthetic-induced postconditioning in the rabbit heart via modulation of phosphatidylinositol-3-kinase/Akt and endothelial nitric oxide synthase signaling. J Cardiovasc Pharmacol 55: 348-357, 2010.

299. Rasola A, Sciacovelli M, Chiara F, Pantic B, Brusilow WS, and Bernardi P. Activation of mitochondrial ERK protects cancer cells from death through inhibition of the permeability transition. Proc Natl Acad Sci U S A 107: 726-731, 2010.

300. Robin E, Guzy RD, Loor G, Iwase H, Waypa GB, Marks JD, Hoek TL, and Schumacker PT. Oxidant Stress during simulated Ischemia primes cardiomyocytes for cell death during reperfusion. J Biol Chem 282: 19133-19143, 2007.

301. Rodriguez-Sinovas A, Boengler K, Cabestrero A, Gres P, Morente M, Ruiz-Meana M, Konietzka I, Miró E, Totzeck A, Heusch G, Schulz R, and Garcia-Dorado D. Translocation of connexin 43 to the inner mitochondrial membrane of cardiomyocytes through the heat shock protein 90dependent TOM pathway and its importance for cardioprotection. Circ Res 99: 93-101, 2006.

302. Roe MT, Messenger JC, Weintraub WS, Cannon CP, Fonarow GC, Dai D, Chen AY, Klein LW, Masoudi FA, McKay C, Hewitt K, Brindis RG, Peterson ED, and Rumsfeld JS. Treatments, trends, and outcomes of acute myocardial infarction and percutaneous coronary intervention. J Am Coll Cardiol 56: 254-263, 2010.

303. Ross AM, Gibbons RJ, Stone GW, Kloner RA, and Alexander RW. A randomized, double-blinded, placebo-controlled multicenter trial of adenosine as an adjunct to reperfusion in the treatment of acute myocardial infarction (AMISTAD-II). J Am Coll Cardiol 45: 1775-1780, 2005.

304. Rottlaender D, Boengler K, Wolny M, Michels G, EndresBecker J, Motloch LJ, Schwaiger A, Buechert A, Schulz R, Heusch G, and Hoppe UC. Connexin 43 acts as a cytoprotective mediator of signal transduction by stimulating mitochondrial $\mathrm{K}_{\mathrm{ATP}}$ channels in mouse cardiomyocytes. $J$ Clin Invest 120: 1441-1453, 2010.

305. Ruiz-Meana M, Abellán A, Miró-Casas E, and GarciaDorado D. Opening of mitochondrial permeability transition pore induces hypercontracture in $\mathrm{Ca}^{2+}$ overloaded cardiac myocytes. Basic Res Cardiol 102: 542-552, 2007.

306. Sack MN. Mitochondrial depolarization and the role of uncoupling proteins in ischemia tolerance. Cardiovasc Res 72: 210-219, 2006.

307. Saitoh S, Kiyooka T, Rocic P, Rogers PA, Zhang C, Swafford A, Dick GM, Viswanathan C, Park Y, and Chilian WM. Redox-dependent coronary metabolic dilation. Am J Physiol Heart Circ Physiol 293: H3720-H3725, 2007.

308. Saotome M, Katoh H, Yaguchi Y, Tanaka T, Urushida T, Satoh H, and Hayashi H. Transient opening of mitochondrial permeability transition pore by reactive oxygen species protects myocardium from ischemia-reperfusion injury. Am J Physiol Heart Circ Physiol 296: H1125-H1132, 2009.
309. Sarkela TM, Berthiaume J, Elfering S, Gybina AA, and Giulivi $C$. The modulation of oxygen radical production by nitric oxide in mitochondria. J Biol Chem 276: 6945-6949, 2001.

310. Sasaki N, Sato T, Ohler A, O'Rourke B, and Marban E. Activation of mitochondrial ATP-dependent potassium channels by nitric oxide. Circulation 101: 439-445, 2000.

311. Sato H, Bolli R, Rokosh GD, Bi Q, Dai S, Shirk G, and Tang $\mathrm{XL}$. The cardioprotection of the late phase of ischemic preconditioning is enhanced by postconditioning via a COX-2-mediated mechanism in conscious rats. Am J Physiol Heart Circ Physiol 293: H2557-H2564, 2007.

312. Scarpulla RC. Transcriptional paradigms in mammalian mitochondrial biogenesis and function. Physiol Rev 88: 611638, 2008.

313. Schaller S, Paradis S, Ngoh GA, Assaly R, Buisson B, Drouot C, Ostuni MA, Lacapere JJ, Bassissi F, Bordet T, Berdeaux A, Jones SP, Morin D, and Pruss RM. TRO40303, a new cardioprotective compound, inhibits mitochondrial permeability transition. J Pharmacol Exp Ther 333: 696-706, 2010.

314. Scheuermann-Freestone M, Madsen PL, Manners D, Blamire AM, Buckingham RE, Styles P, Radda GK, Neubauer $\mathrm{S}$, and Clarke K. Abnormal cardiac and skeletal muscle energy metabolism in patients with type 2 diabetes. Circulation 107: 3040-3046, 2003.

315. Schneider SH, Amorosa LF, Khachadurian AK, and Ruderman NB. Studies on the mechanism of improved glucose control during regular exercise in type 2 (non-insulin dependent) diabetes. Diabetologia 26: 355-360, 1984.

316. Schrauwen P and Hesselink MKC. Oxidative capacity, lipotoxicity and mitochondrial damage in type 2 diabetes. Diabetes 53: 1412-1417, 2004.

317. Schrauwen P. Skeletal muscle uncoupling protein 3 (UCP3): mitochondrial uncoupling protein in search of a function. Curr Opin Clin Nutr Metab Care 5: 265-270, 2002.

318. Schwanke U, Konietzka I, Duschin A, Li X, Schulz R, and Heusch G. No ischemic preconditioning in heterozygous connexin43-deficient mice. Am J Physiol Heart Circ Physiol 283: H1740-H1742, 2002.

319. Scorrano L, Penzo D, Petronilli V, Pagano F, and Bernardi P. Arachidonic acid causes cell death through the mitochondrial permeability transition. Implications for tumor necrosis factor-alpha apoptotic signaling. J Biol Chem 276: 12035-12040, 2001.

320. Shen X, Zheng S, Metreveli N S, and Epstein PN. Protection of cardiac mitochondria by overexpression of MnSOD reduces diabetic cardiomyopathy. Diabetes 55: 798-805, 2006.

321. Shen X, Zheng SR, Thongboonkerd V, Xu M, Pierce WM, Klein JB, and Epstein PN. Cardiac mitochondrial damage and biogenesis in a chronic model of type 1 diabetes Am J Physiol Endocrinol Metab 287: E896-E905, 2004.

322. Shin SM, Cho IJ, and Kim SG. Resveratrol protects mitochondria against oxidative stress through AMP-activated protein kinase-mediated glycogen synthase kinase-3beta inhibition downstream of poly(ADP-ribose)polymeraseLKB1 pathway. Mol Pharmacol 76: 884-895, 2009.

323. Shinmura K, Nagai M, Tamaki K, and Bolli R. Gender and aging do not impair opioid-induced late preconditioning in rats. Basic Res Cardiol 99: 46-55, 2004.

324. Shiva S, Sack MN, Greer JJ, Duranski M, Ringwood LA, Burwell L, Wang X, MacArthur PH, Shoja A, Raghavachari N, Calvert JW, Brookes PS, Lefer DJ, and Gladwin MT. Nitrite augments tolerance to ischemia/reperfusion injury 
via the modulation of mitochondrial electron transfer. J Exp Med 204: 2089-2102, 2007.

325. Shulga N and Pastorino JG. Acyl coenzyme A-binding protein augments bid-induced mitochondrial damage and cell death by activating mu-calpain. J Biol Chem 281: 3082430833, 2006.

326. Sivaraman V, Mudalagiri NR, Di Salvo C, Kolvekar S, Hayward M, Yap J, Keogh B, Hausenloy DJ, and Yellon DM. Postconditioning protects human atrial muscle through the activation of the RISK pathway. Basic Res Cardiol 102: 453-459, 2007.

327. Skyschally A and Heusch G. Reduction of myocardial infarct size by dronedarone in pigs-a pleiotropic action? Cardiovasc Drugs Ther 25: 197-201, 2011.

328. Skyschally A, van Caster P, Boengler K, Gres P, Musiolik J, Schilawa D, Schulz R, and Heusch G. Ischemic postconditioning in pigs: no causal role for RISK activation. Circ Res 104: 15-18, 2009.

329. Skyschally A, van Caster P, Iliodromitis EK, Schulz R, Kremastinos DT, and Heusch G. Ischemic postconditioning: experimental models and protocol algorithms. Basic Res Cardiol 104: 469-483, 2009.

330. Sohal RS and Weindruch R. Oxidative stress, caloric restriction, and aging. Science 273: 59-63, 1996.

331. Solan JL and Lampe PD. Connexin 43 phosphorylation: structural changes and biological effects. Biochem J 419: 261-272, 2009.

332. Sorensson $P$, Saleh N, Bouvier F, Bohm F, Settergren M, Caidahl K, Tornvall P, Arheden H, Ryden L, and Pernow J. Effect of postconditioning on infarct size in patients with ST elevation myocardial infarction. Heart 96: 1710-1715, 2010.

333. Staat P, Rioufol G, Piot C, Cottin Y, Cung TT, L'Huillier I, Aupetit JF, Bonnefoy E, Finet G, Andre-Fouet X, and Ovize M. Postconditioning the human heart. Circulation 112: 2143-2148, 2005.

334. Stowe DF and Camara AK. Mitochondrial reactive oxygen species production in excitable cells: modulators of mitochondrial and cell function. Antioxid Redox Signal 11: 13731414, 2009.

335. St-Pierre J, Drori S, Uldry M, Silvaggi JM, Rhee J, Jager S, Handschin C, Zheng K, Lin J, Yang W, Simon DK, Bachoo $\mathrm{R}$, and Spiegelman BM. Suppression of reactive oxygen species and neurodegeneration by the PGC-1 transcriptional coactivators. Cell 127: 397-408, 2006.

336. Stumpner J, Redel A, Kellermann A, Lotz CA, Blomeyer CA, Smul TM, Kehl F, Roewer N, and Lange M. Differential role of Pim-1 kinase in anesthetic-induced and ischemic preconditioning against myocardial infarction. Anesthesiology 111: 1257-1264, 2009.

337. Suleiman M-S, Halestrap AP, and Griffiths EJ. Mitochondria: a target for myocardial protection. Pharmacol Ther 89: 29-46, 2001.

338. Sun HY, Wang NP, Halkos M, Kerendi F, Kin H, Guyton RA, Vinten-Johansen J, and Zhao ZQ. Postconditioning attenuates cardiomyocyte apoptosis via inhibition of JNK and p38 mitogen-activated protein kinase signaling pathways. Apoptosis 11: 1583-1593, 2006.

339. Sun HY, Wang NP, Kerendi F, Halkos M, Kin H, Guyton RA, Vinten-Johansen J, and Zhao ZQ. Hypoxic postconditioning reduces cardiomyocyte loss by inhibiting ROS generation and intracellular $\mathrm{Ca}^{2+}$ overload. Am J Physiol Heart Circ Physiol 288: H1900-H1908, 2005.

340. Sun J, Morgan M, Shen RF, Steenbergen C, and Murphy E. Preconditioning results in S-nitrosylation of proteins in- volved in regulation of mitochondrial energetics and calcium transport. Circ Res 101: 1155-1163, 2007.

341. Sun J, Steenbergen C, and Murphy E. S-nitrosylation: NOrelated redox signaling to protect against oxidative stress. Antioxid Redox Signal 8: 1693-1705, 2006.

342. Sun JZ, Tang XL, Knowlton AA, Park SW, Qiu Y, and Bolli R. Late preconditioning against myocardial stunning. An endogenous protective mechanism that confers resistance to postischemic dysfunction $24 \mathrm{~h}$ after brief ischemia in conscious pigs. J Clin Invest 95: 388-403, 1995.

343. Szczepanek K, Chen Q, Derecka M, Salloum FN, Zhang Q, Szelag M, Cichy J, Kukreja RC, Dulak J, Lesnefsky EJ, and Larner AC. Mitochondrial-targeted signal transducer and activator of transcription (STAT3) protects against ischemiainduced changes in the electron transport chain and the generation of reactive oxygen species. J Biol Chem 286: 29610-29620, 2011.

344. Szczepanek K, Chen Q, Larner AC, and Lesnefsky EJ. Cytoprotection by the modulation of mitochondrial electron transport chain: the emerging role of mitochondrial STAT3. Mitochondrion 12: 180-189, 2012.

345. Szekeres L. Drug-induced delayed cardiac protection against the effects of myocardial ischemia. Pharmacol Ther 108: 269-280, 2005.

346. Taimor G. Mitochondria as common endpoints in early and late preconditioning. Cardiovasc Res 59: 266-267, 2003.

347. Takagi H, Matsui Y, and Sadoshima J. The role of autophagy in mediating cell survival and death during ischemia and reperfusion in the heart. Antioxid Redox Signal 9: 13731381, 2007.

348. Takano H, Tang XL, Kodani E, and Bolli R. Late preconditioning enhances recovery of myocardial function after infarction in conscious rabbits. Am J Physiol 279: H2372-H2381, 2000.

349. Takashi E, Wang Y, and Ashraf M. Activation of mitochondrial K(ATP) channel elicits late preconditioning against myocardial infarction via protein kinase $C$ signaling pathway. Circ Res 85: 1146-1153, 1999.

350. Tanaka K, Kehl F, Gu W, Krolikowski JG, Pagel PS, Warltier DC, and Kersten JR. Isoflurane-induced preconditioning is attenuated by diabetes. Am J Physiol Heart Circ Physiol 282: H2018-H2023, 2002.

351. Terman A and Brunk UT. Autophagy in cardiac myocyte homeostasis, aging, and pathology. Cardiovasc Res 68: 355365, 2005.

352. Terrell AM, Crisostomo PR, Wairiuko GM, Wang M, Morrell ED, and Meldrum DR. JAK/STAT/SOCS signaling circuits and asociated cytokine-mediated inflammation and hypertrophy in the heart. Shock 26: 226-234, 2006.

353. Thibault H, Piot C, Staat P, Bontemps L, Sportouch C, Rioufol G, Cung TT, Bonnefoy E, Angoulvant D, Aupetit JF, Finet G, André-Fouët X, Macia JC, Raczka F, Rossi R, Itti R, Kirkorian G, Derumeaux G, and Ovize M. Long-term benefit of postconditioning. Circulation 117: 1037-1044, 2008.

354. Thomas DD, Ridnour LA, Isenberg JS, Flores-Santana W, Switzer CH, Donzelli S, Hussain P, Vecoli C, Paolocci N, Ambs S, Colton CA, Harris CC, Roberts DD, and Wink DA. The chemical biology of nitric oxide: implications in cellular signaling. Free Radic Biol Med 45: 18-31, 2008.

355. Tian Y, Zhang W, Xia D, Modi P, Liang D, and Wei M. Postconditioning inhibits myocardial apoptosis during prolonged reperfusion via a JAK2-STAT3-Bcl-2 pathway. J Biomed Sci 18: 53, 2011 
356. Tong $\mathrm{H}$, Imahashi $\mathrm{K}$, Steenbergen $\mathrm{C}$, and Murphy E. Phosphorylation of glycogen synthase kinase- $3 \beta$ during preconditioning through a phosphatidylinositol-3-kinasedependent pathway is cardioprotective. Circ Res 90: 374376, 2002.

357. Totzeck A, Boengler K, Van de SA, Konietzka I, Gres P, Garcia-Dorado D, Heusch G, and Schulz R. No impact of protein phosphatases on connexin 43 phosphorylation in ischemic preconditioning. Am J Physiol Heart Circ Physiol 295: H2106-H2112, 2008.

358. Tritto I and Ambrosio G. Role of oxidants in the signaling pathway of preconditioning. Antioxid Redox Signal 3: 3-10, 2001.

359. Tsang A, Hausenloy DJ, Mocanu MM, Carr RD, and Yellon DM. Preconditioning the diabetic heart: the importance of Akt phosphorylation. Diabetes 54: 2360-2364, 2005.

360. Tsutsumi Y, Matsubara H, Masaki H, Kurihara H, Murasawa S, Takai S, Miyazaki M, Nozawa Y, Ozono R, Nakagawa K, Miwa T, Kawada N, Mori Y, Shibasaki Y, Tanaka Y, Fujiyama S, Koyama Y, Fujiyama A, Takahashi $\mathrm{H}$, and Iwasaka T. Angiotensin II type 2 receptor overexpression activates the vascular kinin system and causes vasodilation. J Clin Invest 104: 925-935, 1999.

361. Tsutsumi YM, Yokoyama T, Horikawa Y, Roth DM, and Patel HH. Reactive oxygen species trigger ischemic and pharmacological postconditioning: in vivo and in vitro characterization. Life Sci 81: 1223-1227, 2007.

362. Turko IV and Murad F. Protein nitration in cardiovascular diseases. Pharmacol Rev 54: 619-634, 2002.

363. Vanden Hoek TL, Becker LB, Shao Z-H, Li C-Q, and Schumacker PT. Preconditioning in cardiomyocytes protects by attenuating oxidant stress at reperfusion. Circ Res 86: 541-548, 2000.

364. Vanden Hoek TL, Li C, Shao Z, Schumacker PT, and Becker LB. Significant levels of oxidants are generated by isolated cardiomyocytes during ischemia prior to reperfusion. J Mol Cell Cardiol 29: 2571-2583, 1997.

365. Vanden Hoek TL, Shao Z, Li C, Schumacker PT, and Becker LB. Mitochondrial electron transport can become a significant source of oxidative injury in cardiomyocytes. J Mol Cell Cardiol 29: 2441-2450, 1997.

366. Vegh A, Komori S, Szekeres L, and Parratt JR. Antiarrhythmic effects of preconditioning in anaesthetised dogs and rats. Cardiovasc Res 26: 487-495, 1992.

367. Vinten-Johansen J, Buckberg GD, Okamoto F, Rosenkranz ER, Bugyi H, and Leaf J. Superiority of surgical versus medical reperfusion after regional ischemia. J Thorac Cardiovasc Surg 92: 525-534, 1986.

368. Vinten-Johansen J, Granfeldt A, Mykytenko J, Undyala VV, Dong Y, and Przyklenk K. The multi-dimensional physiological responses to postconditioning. Antioxid Redox Signal 14: 791-810, 2011.

369. Vinten-Johansen J, Zhao ZQ, Zatta AJ, Kin H, Halkos ME, and Kerendi F. Postconditioning. A new link in nature's armor against myocardial ischemia reperfusion injury. Basic Res Cardiol 295: 295-310, 2005.

370. Viollet B, Andreelli F, Jørgensen SB, Perrin C, Geloen A, Flamez D, Mu J, Wagner C, Kloeting I, Strasser RH, and Weinbrenner C. Cardioprotection by postconditioning is lost in WOKW rats with metabolic syndrome: role of glycogen synthase kinase 3beta. J Cardiovasc Pharmacol 52: 430-437, 2008.

371. Wagner C, Tillack D, Simonis G, Strasser RH, and Weinbrenner $\mathrm{C}$. Ischemic postconditioning reduces infarct size of the in vivo rat heart: role of PI3-K, mTOR, GSK-3beta, and apoptosis. Mol Cell Biochem 339: 135-147, 2010.

372. Wang W, Fang H, Groom L, Cheng A, Zhang W, Liu J, Wang X, Li K, Han P, Zheng M, Yin J, Wang W, Mattson MP, Kao JP, Lakatta EG, Sheu SS, Ouyang K, Chen J, Dirksen RT, and Cheng H. Superoxide flashes in single mitochondria. Cell 134: 279-290, 2008.

373. Wegrzyn J, Potla R, Chwae YJ, Sepuri NB, Zhang Q, Koeck T, Derecka M, Szczepanek K, Szelag M, Gornicka A, Moh A, Moghaddas S, Chen Q, Bobbili S, Cichy J, Dulak J, Baker DP, Wolfman A, Stuehr D, Hassan MO, Fu XY, Avadhani N, Drake JI, Fawcett P, Lesnefsky EJ, and Larner AC. Function of mitochondrial Stat3 in cellular respiration. Science 323: 793-797, 2009.

374. Weidenbach R, Schulz R, Gres P, Behrends M, Post H, and Heusch $G$. Enhanced reduction of myocardial infarct size by combined ACE inhibition and AT1-receptor antagonism. Br J Pharmacol 131: 138-144, 2000.

375. Wenzel P, Schuhmacher S, Kienhofer J, Muller J, Hortmann M, Oelze M, Schulz E, Treiber N, Kawamoto T, Scharffetter-Kochanek K, Münzel T, Bürkle A, Bachschmid MM, and Daiber A. Manganese superoxide dismutase and aldehyde dehydrogenase deficiency increase mitochondrial oxidative stress and aggravate age-dependent vascular dysfunction. Cardiovasc Res 80: 280-289, 2008.

376. Williams SD and Gottlieb RA. Inhibition of mitochondrial calcium-independent phospholipase A2 (iPLA2) attenuates mitochondrial phospholipid loss and is cardioprotective. Biochem J 362: 23-32, 2002.

377. Wisløff U, Najjar SM, Ellingsen O, Haram PM, Swoap S, AlShare Q, Fernström M, Rezaei K, Lee SJ, Koch LG, and Britton SL. Cardiovascular risk factors emerge after artificial selection for low aerobic capacity. Science 307: 418-420, 2005.

378. Xu C, Yi C, Wang H, Bruce IC, and Xia Q. Mitochondrial nitric oxide synthase participates in the septic shock myocardial depression by NO overproduction and mitochondrial permeability transition pore opening. Shock 37: 110-115, 2012.

379. Xu M, Zhao YT, Song Y, Hao TP, Lu ZZ, Han QD, Wang SQ, and Zhang YY. Alpha1-adrenergic receptors activate AMP-activated protein kinase in rat hearts. Sheng Li Xue Bao 59: 175-182, 2007.

380. Yan L, Sadoshima J, Vatner DE, and Vatner SF. Autophagy in ischemic preconditioning and hibernating myocardium. Autophagy 5: 709-712, 2009.

381. Yang B, Larson DF, and Watson RR. Modulation of iNOS activity in age-related cardiac dysfunction. Life Sci 75: 655667, 2004.

382. Yellon DM and Downey JM. Preconditioning the myocardium: from cellular physiology to clinical cardiology. Physiol Rev 83: 1113-1151, 2003.

383. Yellon DM and Hausenloy DJ. Myocardial reperfusion injury. N Engl J Med 357: 1121-1135, 2007.

384. Yellon DM and Hausenloy DJ. Realizing the clinical potential of ischemic preconditioning and postconditioning. Nat Clin Pract Cardiovasc. Med 2: 568-575, 2005.

385. Yin Z, Gao H, Wang H, Li L, Di C, Luan R, and Tao L. Ischaemic post-conditioning protects both adult and aged Sprague-Dawley rat heart from ischaemia-reperfusion injury through the phosphatidylinositol 3-kinase-AKT and glycogen synthase kinase-3beta pathways. Clin Exp Pharmacol Physiol 36: 756-763, 2009.

386. Youle RJ and Strasser A. The BCL-2 protein family: opposing activities that mediate cell death. Nat Rev Mol Cell Biol 9: 47-59, 2008. 
387. Yue Y, Qin Q, Cohen MV, Downey JM, and Critz SD. The relative order of $\mathrm{mK}(\mathrm{ATP})$ channels, free radicals and p38 MAPK in preconditioning's protective pathway in rat heart. Cardiovasc Res 55: 681-689, 2002.

388. Zatta AJ, Kin H, Lee G, Wang N, Jiang R, Lust R, Reeves JG, Mykytenko J, Guyton RA, Zhao ZQ, and Vinten-Johansen $\mathrm{J}$. Infarct-sparing effect of myocardial postconditioning is dependent on protein kinase $\mathrm{C}$ signalling. Cardiovasc Res 70: 315-324, 2006

389. Zhang DX, Chen YF, Campbell WB, Zou AP, Gross GJ, and Li PL. Characteristics and superoxide-induced activation of reconstituted myocardial mitochondrial ATP-sensitive potassium channels. Circ Res 89: 1177-1183, 2001.

390. Zhang Y, Xia Z, La Cour KH, and Ren J. Activation of Akt rescues endoplasmic reticulum stress-impaired murine cardiac contractile function via glycogen synthase kinase$3 \beta$-mediated suppression of mitochondrial permeation pore opening. Antioxid Redox Signal 15: 2407-2424, 2011.

391. Zhang YY, Xu AM, Nomen M, Walsh M, Keaney JF, Jr., Turko IV, Murad F, and Loscalzo J. Nitrosation of tryptophan residue(s) in serum albumin and model dipeptides. Biochemical characterization and bioactivity. J Biol Chem 271: 14271-14279, 1996.

392. Zhao ZQ and Vinten-Johansen J. Postconditioning: reduction of reperfusion-induced injury. Cardiovasc Res 70: 200211, 2006.

393. Zhao ZQ, Corvera JS, Halkos ME, Kerendi F, Wang NP, Guyton RA, and Vinten-Johansen J. Inhibition of myocardial injury by ischemic postconditioning during reperfusion: comparison with ischemic preconditioning. Am J Physiol Heart Circ Physiol 285: H579-H588, 2003.

394. Zhu M, Feng J, Lucchinetti E, Fischer G, Xu L, Pedrazzini T, Schaub MC, and Zaugg M. Ischemic postconditioning protects remodeled myocardium via the PI3K-PKB/Akt reperfusion injury salvage kinase pathway. Cardiovasc Res 72: 152-162, 2006.

395. Zmijewski JW, Banerjee S, Bae H, Friggeri A, Lazarowski ER, and Abraham E. Exposure to hydrogen peroxide induces oxidation and activation of AMP-activated protein kinase. J Biol Chem 285: 33154-33164, 2010.

396. Zorov DB, Filburn CR, Klotz LO, Zweier JL, and Sollott SJ. Reactive oxygen species (ROS)-induced ROS release: a new phenomenon accompanying induction of the mitochondrial permeability transition in cardiac myocytes. J Exp Med 192: 1001-1014, 2000.

397. Zorov DB, Juhaszova M, and Sollott SJ. Mitochondrial ROS-induced ROS release: an update and review. Biochim Biophys Acta 1757: 509-517, 2006.

398. Zorov DB, Juhaszova M, Yaniv Y, Nuss HB, Wang S, and Sollott SJ. Regulation and pharmacology of the mitochondrial permeability transition pore. Cardiovasc Res 83: 213225, 2009.

399. Zou M, Martin C, and Ullrich V. Tyrosine nitration as a mechanism of selective inactivation of prostacyclin synthase by peroxynitrite. Biol Chem 378: 707-713, 1997.

400. Zou MH, Kirkpatrick SS, Davis BJ, Nelson JS, Wiles WG, 4th, Schlattner U, Neumann D, Brownlee M, Freeman MB, and Goldman MH. Activation of the AMP-activated protein kinase by the anti-diabetic drug metformin in vivo. Role of mitochondrial reactive nitrogen species. J Biol Chem 279: 43940-43951, 2004.

401. Zweier JL, Wang P, and Kuppusamy P. Direct measurement of nitric oxide generation in the ischemic heart using electron paramagnetic resonance spectroscopy. J Biol Chem 270: 304-307, 1995.

Address correspondence to: Dr. Pasquale Pagliaro

Dipartimento di Scienze Cliniche e Biologiche Università di Torino

Regione Gonzole 10 10043 Orbassano (TO)

Italy

E-mail: pasquale.pagliaro@unito.it

Date of first submission to ARS Central, December 6, 2011; date of final revised submission, May 15, 2012; date of acceptance, June 5, 2012.

\section{Abbreviations Used}

$\Delta \psi \mathrm{m}=$ mitochondrial membrane potential

AICAR $=$ aminoimidazole carboxamide ribonucleotide

$\mathrm{AIF}=$ apoptosis-inducing factor

ALDH $=$ aldehyde dehydrogenase

$\mathrm{AMI}=$ acute myocardial infarction

$\mathrm{AMPK}=\mathrm{AMP}$-activated protein kinase

$\mathrm{ANT}=$ adenine nucleotide transporter

APAF1 $=$ apoptotic peptidase-activating factor 1

$\mathrm{ATP}=$ adenosine triphosphate

Bcl-2 = B-cell lymphoma-2

$\mathrm{cGMP}=$ cyclic guanosine monophosphate

$\mathrm{COX}=$ cyclooxygenase

$\mathrm{CsA}=$ cyclosporine $\mathrm{A}$

$\mathrm{C} \times 43=$ connexin -43

Cyp-D $=$ cyclophilin D

Cyt $\mathrm{c}=$ cytochrome $\mathrm{c}$

$\mathrm{DAG}=$ diacylglycerol

$\mathrm{DDP}=$ deafness dystonia protein

Drp1 $=$ dynamin-related protein 1

eNOS $=$ endothelial nitric oxide synthase

$\mathrm{ERK}=$ extracellular signal-regulated kinase

$\mathrm{ETC}=$ electron transport chain

GSK $=$ glycogen synthase kinase

$\mathrm{GSNO}=$ S-nitrosoglutathione

$\mathrm{H}_{2} \mathrm{O}_{2}=$ hydrogen peroxide

$\mathrm{HKs}=$ hexokinases

$\mathrm{HNE}=$ 4-hydroxynonenal

Hsp90 = heat shock protein 90

IAPs $=$ inhibitor of apoptosis proteins

$\mathrm{IMM}=$ inner mitochondrial membrane

iNOS $=$ inducible nitric oxide synthase

$\mathrm{I} / \mathrm{R}=$ ischemia/reperfusion

$\mathrm{JAK}=$ Janus kinase

$\mathrm{K}_{\mathrm{ATP}}=\mathrm{ATP}$-sensitive potassium channels

$\mathrm{KO}=$ knockout

$\mathrm{MAC}=$ mitochondrial apoptosis-induced channel

MAOs $=$ monoamine oxidases

MAPK $=$ mitogen-activated protein kinase

$\mathrm{mCx} 43=$ mitochondrial $\mathrm{Cx} 43$

$\mathrm{Mfn}=$ mitofusin 


\section{Abbreviations Used (Cont.)}

$\mathrm{mK}_{\mathrm{ATP}}=$ mitochondrial ATP-sensitive potassium channels

$\mathrm{MnSOD}=$ manganese superoxide dismutase

$\mathrm{MPG}=$ mercaptopropionylglycine

$\mathrm{mPTP}=$ mitochondrial permeability transition pore

$\mathrm{MS}=$ metabolic syndrome

$\mathrm{NAC}=\mathrm{N}$-acetylcysteine

$\mathrm{NCE}=\mathrm{Na}^{+} / \mathrm{Ca}^{2+}$ exchanger

$\mathrm{NF} \kappa \mathrm{B}=$ nuclear factor $\kappa \mathrm{B}$

$\mathrm{NHE}=\mathrm{Na}^{+} / \mathrm{H}^{+}$exchanger

$\mathrm{nNOS}=$ neuronal nitric oxide synthase

$\mathrm{NO}^{\bullet}=$ nitric oxide

$\mathrm{NOS}=$ nitric oxide synthase

$\mathrm{O}_{2}{ }^{-\bullet}=$ superoxide anion

$\mathrm{OH}^{\bullet}=$ hydroxyl radical

$\mathrm{OMM}=$ outer mitochondrial membrane

$\mathrm{ONOO}^{-}=$peroxynitrite

$\mathrm{PCI}=$ percutaneous coronary intervention

$\mathrm{PDH}=$ pyruvate dehydrogenase

PGC $=$ peroxisome proliferator-activated receptor $\gamma$ coactivator

$\mathrm{Pi}=$ inorganic phosphate

PI3K = phosphatidylinositol 3-kinase

$\mathrm{PKA}=$ protein kinase $\mathrm{A}$

$\mathrm{PKB} / \mathrm{Akt}=$ protein kinase $\mathrm{B}$
PKC $=$ protein kinase $C$

$\mathrm{PKG}=$ protein kinase $\mathrm{G}$

PMA = phorbol 12-myristate 13-acetate

PostC $=$ postconditioning

PPARs $=$ peroxisome proliferator-activated receptors

PreC $=$ preconditioning

$\mathrm{PS}=$ phosphatidylserine

RIRR $=$ ROS-induced ROS release

RISKs = reperfusion injury salvage kinases

RNS $=$ reactive nitrogen species

ROS $=$ reactive oxygen species

$\mathrm{SAFE}=$ survivor-activating factor enhancement

$\mathrm{SNO}=$ S-nitrosylation

$\mathrm{STAT}=$ signal transducer and activator of transcription

STEMI $=$ ST segment elevation myocardial infarction

SWOP $=$ second window of protection

TIGAR $=$ TP53-induced glycolysis and apoptosis regulator

$\mathrm{TNF}=$ tumor necrosis factor

Tom20 $=$ outer membrane translocase 20

$\mathrm{TSPO}=$ mitochondrial translocator protein $18 \mathrm{kDa}$

$\mathrm{UCPs}=$ uncoupling proteins

$\mathrm{VDAC}=$ voltage-dependent anion channel $\mathrm{VO}_{2}=$ oxygen consumption 